\title{
The limits of evolutionary convergence in sympatry:
}

\section{reproductive interference and developmental constraints}

\section{leading to local diversity in warning traits}

\author{
Ludovic Maisonneuve ${ }^{1, *}$ \\ Marianne Elias ${ }^{1}$ \\ Charline Smadi ${ }^{2}$ \\ Violaine Llaurens ${ }^{1}$
}

1. Institut de Systématique, Evolution, Biodiversité (ISYEB), Muséum national d'Histoire naturelle, CNRS, Sorbonne Université, EPHE, Université des Antilles CP 50, 57 rue Cuvier, 75005 Paris, France;

2. Univ. Grenoble Alpes, INRAE, LESSEM, F-38402 St-Martin-d'Hères, France and Univ. Grenoble Alpes, CNRS, Institut Fourier, 38610 Gières, France;

* Corresponding author; e-mail: ludovic.maisonneuve.2015@polytechnique.org.

Keywords: Mimicry, Reproductive interference, Convergence, Imperfect mimicry, Quantitative genetics.

Manuscript type: Article. 


\begin{abstract}
Mutualistic interactions between defended species represent a striking case of evolutionary convergence in sympatry, driven by the increased protection against predators brought by mimicry in warning traits. However, such convergence is often limited: sympatric defended species frequently display different or imperfectly similar warning traits. The phylogenetic distance between sympatric species may indeed prevent evolution towards the exact same signal. Moreover, warning traits are also involved in mate recognition, so that trait convergence might result in heterospecific courtship and mating. Here, we develop a mathematical model to investigate the strength and direction of evolution of warning trait in defended species with different ancestral traits. Specifically, we determine the effect of phenotypic distances between ancestral trait states of sympatric defended species, and costs of heterospecific sexual interactions on imperfect mimicry and trait divergence. Our analytical results confirm that reproductive interference limits the convergence of warning traits, leading to either complete divergence, or imperfect mimicry, but only between species with different ancestral traits. Surprisingly, our model pinpoints that, depending on the relative species densities, the mimic/model relationships between sympatric species can be inverted. Our study suggests that reproductive interference may promote the emergence of novel warning traits, enhancing mimetic diversity.
\end{abstract}




\section{Introduction}

Evolutionary convergence of traits between species is often promoted by natural selection, but trait similarity between species may be impaired by contrasted developmental and ecological ski, 1992)) or even different warning traits (e.g. (Beccaloni, 1997)) (Briolat et al., 2019). 
The level of trait convergence between sympatric species can vary because of other developindeed frequently involved in species recognition (Jiggins et al., 2001; Kronforst et al., 2006; Mer- 
rill et al., 2014; Naisbit et al., 2001), leading to risk of confusion during sexual interactions. Species

sharing similar warning traits may thus be exposed to substantial reproductive interference incurring fitness costs during mate acquisition due to interspecific interactions, including heterospecific courtship and mating as well as heterospecific male rivalry (Gröning and Hochkirch, 2008). Such reproductive interference has been observed between species sharing similar warning traits (in Heliconius butterfly species for instance (Estrada and Jiggins, 2008)). However, empirical studies precisely estimating the level of reproductive interference in sympatric species are scarce. Pheromone differences between mimetic species have been documented to limit the rate of erroneous mating (see Darragh et al. (2017); González-Rojas et al. (2020) for empirical examples in Heliconius butterflies). However, the pheromones of day-flying butterflies usually act as shortdistance cues that may be perceived only during the courtship (Mérot et al., 2015). Females deceived by the color pattern of the heterospecific males may have spent time and energy or may need to deploy substantial efforts to avoid heterospecific mating. Therefore, females may still suffer from cost associated to reproductive interference, even if females refuse mating with heterospecific males. When females are courted by heterospecific males displaying their preferred cue before being rejected, this results in increased costs associated with mate searching in males (i.e. signal jamming in (Gröning and Hochkirch, 2008)).

Reproductive interference can generate reproductive character displacement (Gröning and Hochkirch, 2008; Kyogoku, 2015), whereby reproductive traits are more dissimilar between species in sympatric than in allopatric populations (Brown and Wilson, 1956). Such reproductive character displacement may thus impair convergence driven by mutualistic interactions. Theoretical studies have investigated how the evolution of female preferences may promote reproductive character displacement in males (McPeek and Gavrilets, 2006; Yamaguchi and Iwasa, 2013): reproductive interference costs are predicted to favor the divergence of female preferences, therefore promoting the divergence of male reproductive traits through sexual selection. Nevertheless, numerical simulations show that the effect of reproductive interference on trait divergence is not straightforward, when those traits are under strong selection promoting convergence (Boussens- 
Dumon and Llaurens, 2021). However, the outcomes of these antagonistic selective forces might range from trait divergence to full convergence, through limited convergence and cannot be investigated in models assuming only discrete and well-differentiated warning traits, calling for a theoretical framework providing general expectations on the gradual evolution of convergent traits.

Here, we thus investigate the ecological factors limiting the convergence of traits involved in mutualistic interactions, by building a mathematical model that describes the evolution of quantitative traits in a focal species involved in mutualistic interactions within a fixed community of defended species. We specifically study the evolution of (1) the quantitative trait $t$ involved in mutualistic interaction, displayed in both males and females and (2) the preference $p$ of females which value indicates male trait value preferred by the females. We assume that individuals gain protection against predators from the similarity of their warning trait $t$ toward the trait displayed in the local community of defended species. However, trait similarity between species generates fitness costs for females via reproductive interference (McPeek and Gavrilets, 2006; Yamaguchi and Iwasa, 2013). We also investigate the effect of developmental and selective constraints by testing the effect of the ancestral trait value $t_{a}$ on trait convergence. Using weak selection approximation (Barton and Turelli, 1991; Kirkpatrick et al., 2002), we obtain equations describing the evolution of the mean trait and mean preference values in the focal species. We then use analytical results and numerical analyses to investigate the effect of reproductive interference on the convergence of trait depending on different ecological factors. Finally, to specifically investigate the effect of reproductive interference and of ancestral trait values on the direction of advergence, we extend our model to two interacting species that exhibit different densities, individual levels of defense and ancestral trait values, and explore the joint evolution of displayed trait and female preference in both species. 


\section{Evolution of warning trait toward a fixed community of defended}

\section{species}

\section{Methods}

We consider a single species (refered to as focal species hereafter) living in sympatry with a in Equation (7)) the contribution of a mating between a male with trait $t_{m}$ and a female with 
preference $p_{f}$ to the next generation. We also compute $W_{R I}\left(p_{f}\right)$ (defined after in Equation (12) the female fitness component describing fitness reduction due to RI. Following (Kirkpatrick et al., 2002) the reproductive success $W\left(t_{m}, t_{f}, p_{f}\right)$ stemming from a mated pair of a male with trait $t_{m}$ and a female with trait $t_{f}$ and preference $p_{f}$ is given by:

$$
W\left(t_{m}, t_{f}, p_{f}\right)=W_{n s}\left(t_{m}\right) W_{r}\left(t_{m}, p_{f}\right) W_{n s}\left(t_{f}\right) W_{R I}\left(p_{f}\right)
$$

Using the Price's theorem (see Rice (2004) for instance), we can approximate the change in the acting on the trait $t$ and the preference $p$, respectively and are given by:

$$
\begin{aligned}
& \beta_{t}:=\frac{\partial}{\partial t_{m}} \ln W\left(t_{m}, t_{f}, p_{f}\right)+\left.\frac{\partial}{\partial t_{f}} \ln W\left(t_{m}, t_{f}, p_{f}\right)\right|_{\left(t_{m}, t_{f}, p_{f}\right)=(\bar{t}, \bar{t}, \bar{p})}, \\
& \beta_{p}:=\left.\frac{\partial}{\partial p_{f}} \ln W\left(t_{m}, t_{f}, p_{f}\right)\right|_{\left(t_{m}, t_{f}, p_{f}\right)=(\bar{t}, \bar{t}, \bar{p})} .
\end{aligned}
$$

We assume weak natural and sexual selective pressures (Iwasa et al., 1991; Pomiankowski and Iwasa, 1993), i.e. that the difference of fitness between different individuals is at maximum of order $\varepsilon$, with $\varepsilon$ being small. Under this hypothesis, genetic correlations generated by selection and non-random mating quickly reach equilibrium (Nagylaki, 1993) and can thus be approximated by their equilibrium values. Weak selection hypothesis also implies that the variance of traits and preference is low (Iwasa et al., 1991).

Following (Iwasa et al., 1991), we assume that $G_{t}$ and $G_{p}$ are positive constants maintained by an equilibrium between selection and recurrent mutations. Under weak selection, the genetic covariance between $t$ and $p$ can be approximated by (see Appendix 1.3):

$$
C_{t p}=a G_{t} G_{p}
$$


Because under weak selection $G_{t}$ and $G_{p}$ are small, $C_{t p}$ is small in comparison with $G_{t}$ and $G_{p}$, allowing us to approximate the change in the mean values of trait and preference by:

$$
\left(\begin{array}{c}
\Delta \bar{t} \\
\Delta \bar{p}
\end{array}\right) \approx \frac{1}{2}\left(\begin{array}{cc}
G_{t} & 0 \\
0 & G_{p}
\end{array}\right)\left(\begin{array}{l}
\beta_{t} \\
\beta_{p}
\end{array}\right)=\frac{1}{2}\left(\begin{array}{c}
G_{t} \beta_{t} \\
G_{p} \beta_{p}
\end{array}\right) .
$$

\section{Reproduction}

The contribution to the next generation of a mating between a male with trait $t$ and a female with preference $p$ is given by

$$
W_{r}(t, p)=\exp \left[-a(p-t)^{2}\right]
$$

where female choosiness $a$, assumed constant among females, quantifies how much females reject males with a non-preferred trait. We assume weak preference, in line with the previouslydescribed weak selection hypothesis, i.e. $a$ being of order $\varepsilon$, with $\varepsilon$ small, and that $a>0$.

\section{Ancestral trait value}

The phenotypic evolution in the focal species also strongly depends on ancestral trait value $t_{a}$, and on the distance between this ancestral value and the trait value displayed in the local mimicry ring.

Phenotypic evolution in the focal species away from this ancestral trait is limited by developmental as well as selective constraints, specific to the focal species. The natural selection exerted on an individual displaying a trait value $t$ then depends on (1) natural selection due to mimetic protection against predation, as well as (2) departure from the ancestral trait value $t_{a}$, inducing a selective and developmental cost $s$. It is thus given by:

$$
W_{n s}(t)=W_{\text {pred }}(t) \exp \left[-s\left(t-t_{a}\right)^{2}\right]
$$

where $W_{\text {pred }}$ is the fitness component due to predation (see Equation (11) below). We assume a selective coefficient $s$ of order $\varepsilon^{2}$, because we focus on the specific case where selective pressures 
due to selective constraints, predation and reproductive interference are of the same order of magnitude (see Appendix 1.1).

Predation depending on the level of mimicry of trait $t$.

The evolution of the trait $t$, expressed by males and females from the focal species, is strongly influenced by the trait displayed by the individuals from the local mimicry ring (mean value $\bar{t}^{\prime}$ ). Müllerian mimicry indeed generates positive density-dependent selection (Benson, 1972; Chouteau et al., 2016; Mallet and Barton, 1989), due to predator learning. This density-dependence is non linear and is often modelled as an hyperbolic decrease in mortality (see (Joron and Iwasa, 2005; Llaurens et al., 2013) for example). The density-dependence is modulated by the individual defense level $\lambda$, assumed constant among individuals of the focal species, shaping predator deterrence: the higher the defense, the quicker predators learn. We note $\bar{\lambda}^{\prime}$ the mean defense level of an individual in the local mimicry ring. The protection gained against predators then depends on the level of resemblance among defended prey, as perceived by predator and the number of individuals sharing the same trait. The level of protection gained by an individual with trait $t$ because of its resemblance with other individuals is given by:

$$
\mathcal{D}(t)=\overbrace{\int_{\tau} \lambda N f(\tau) \exp \left[-b(t-\tau)^{2}\right] \mathrm{d} \tau}^{\begin{array}{c}
\text { protection gained by resemblance } \\
\text { with individuals of the focal species }
\end{array}}+\overbrace{\int_{t^{\prime}} \lambda^{\prime} N^{\prime} g\left(t^{\prime}\right) \exp \left[-b\left(t-t^{\prime}\right)^{2}\right] \mathrm{d} t^{\prime}}^{\begin{array}{c}
\text { protection gained by resemblance } \\
\text { with individuals of the local mimicry ring species }
\end{array}},
$$

where $N$ and $N^{\prime}$ are the densities of individuals in the focal species and in the local mimicry ring, respectively and $\exp \left[-b(t-\tau)^{2}\right]$ describes how much predators perceive the trait values $t$ and $\tau$ as similar. The predator discrimination coefficient $b$ thus quantifies how much predators discriminate different trait values. We assume that this parameter is of the same order of magnitude as female choosiness $a$ (i.e of order $\varepsilon$ ). $f$ and $g$ are the distribution of traits in the focal species and in the local mimicry ring species respectively. Assuming that the distribution of traits has a low variance within both the focal species and the local mimicry ring leads to the following approximation 

of an individual displaying the trait value $t$ is given by:

$$
W_{\text {pred }}(t)=1-\frac{d}{\mathcal{D}(t)}
$$

where $\exp \left[-b\left(t-\bar{t}^{\prime}\right)^{2}\right]\left(\right.$ resp. $\left.\exp \left[-b(t-\bar{t})^{2}\right]\right)$ describes how much predators perceive the trait values $t$ and $\bar{t}^{\prime}$ (resp. $\bar{t}$ ) as similar.

\section{Cost induced by reproductive interference.}

Because males of the local mimicry ring may resemble males of the focal species, females will suffer from reproductive interference generated by erroneous mating attempts with heterospecific males (see (Gröning and Hochkirch, 2008) for a review of reproductive interference costs). The risk of heterospecific mating depends on the relative densities of heterospecific and conspecific males. The cost of reproductive interference is thus assumed to be density-dependent in the model. We assume a balanced sex-ratio within both the focal species and the local mimicry ring. However, we also consider the capacity of females to recognize conspecific males using alternative cues (pheromones for example). In the model, the investment of females in interspecific mating interaction is captured by the parameter $c_{R I} \in[0,1]$. This cost of reproductive interference incurred to females can be reduced when female choice is also based on alternative cues differing between mimetic species. Using Equation (1b) in (Yamaguchi and Iwasa, 2013) the fitness of a 
female with preference $p$ is modulated by:

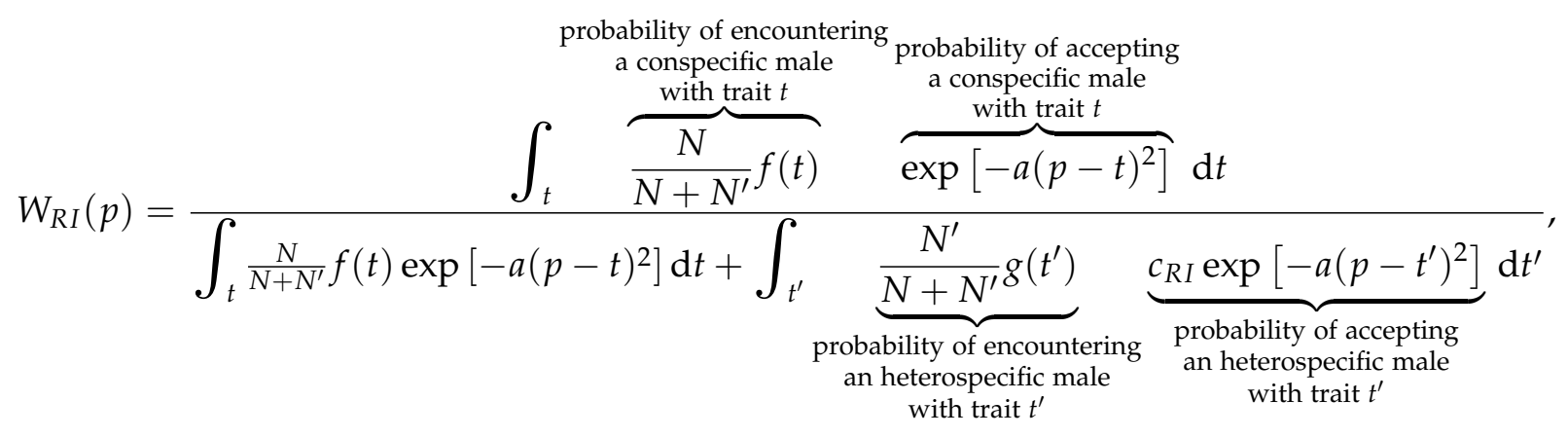

where $c_{R I}$ is the strength of RI, assumed of order $\varepsilon$, with $\varepsilon$ small, in line with the weak selection hypothesis.

Assuming that the distribution of traits has a low variance within both the focal species and the local mimicry ring leads as before to the following approximation:

$$
W_{R I}(p)=\frac{\exp \left[-a(p-\bar{t})^{2}\right] N}{\exp \left[-a(p-\bar{t})^{2}\right] N+c_{R I} \exp \left[-a\left(p-\bar{t}^{\prime}\right)^{2}\right] N^{\prime}}
$$

All variables and parameters used in the model are summed up in Table 1.

\section{Model exploration.}

We assume that the focal species is ancestrally not in contact with the local mimicry ring displaying the ancestral trait $t_{a}$. We also assume that mean female preference value is initially equal to mean trait value. At the initial time, we assume that the focal species enters in contact with the local mimicry ring. The dynamics of trait and preference values then follow Equation (6).

Analytical resolution assuming weak female choosiness $(a=O(\varepsilon))$ and predator discrimination $(b=O(\varepsilon)$ ). Assuming that $a$ and $b$ are of order $\varepsilon$ and $s$ is of order $\varepsilon^{2}$ (hypothesis $H_{w}$ ), we analytically determine the mean trait and preference values in the focal species at equilibrium $\left(\bar{t}^{*}\right.$ and $\left.\bar{p}^{*}\right)$. To identify the conditions enabling advergence toward the local mimicry ring, we estimate $\left|\bar{t}^{*}-\bar{t}^{\prime}\right|$, i.e. the phenotypic distance between the mean trait within the focal species and the mean trait displayed in the local mimicry ring. 
Table 1: Description of variables and parameters used in the model assuming a fixed local mimicry ring

\begin{tabular}{ll} 
Abbreviation & Description \\
\hline $\bar{t} / \bar{p}$ & Mean trait/preference value in the focal species \\
$G_{t} / G_{p}$ & Genetic variance of trait $t /$ preference $p$ \\
$C_{t p}$ & Genetic covariance between trait $t$ and preference $p$ \\
$\beta_{t} / \beta_{p}$ & Selection coefficient on trait $t /$ preference $p$ \\
$\bar{t}^{\prime}$ & Mean trait value in the mimetic community \\
$a$ & Female choosiness \\
$s$ & Strength of selective and developmental constraints promoting the ancestral trait value $t_{a}$ \\
$t_{a}$ & ancestral trait value in the focal species \\
$d$ & Basal predation rate \\
$b$ & Predator discrimination coefficient \\
$\lambda$ & defense level carried out by individuals of the focal species \\
$\bar{\lambda}^{\prime}$ & Mean defense level of an individual of the local mimicry ring \\
$N / N^{\prime}$ & Density of the focal species /local mimicry ring \\
$c_{R I}$ & Strength of reproductive interference \\
\hline
\end{tabular}
ring, we then compute the discrepancy between trait and preference values in the focal species $\left|\bar{t}^{*}-\bar{p}^{*}\right|$.

Model exploration assuming strong female choosiness $(a=O(1))$ and predator discrimination $(b=O(1))$.

Because the stringency of females choice, as well as the discrimination behavior of predators, are key drivers of the advergence toward the trait displayed in the local mimicry ring, we then explore large values of female choosiness and predator discrimination, i.e. $a=O(1)$ and $b=O(1)$. Under such assumptions, the effects of predation and reproductive interference on the changes in 
the mean trait and preference are of order $\varepsilon$. We also assume that $s$ is of order $\varepsilon$, because if $s$ is of

We study the evolution of the mean values of trait and preference in the focal species, described by Equation (6). When

$$
c_{R I}<\frac{N}{a N^{\prime}}\left(\frac{4 b d \bar{\lambda}^{\prime} N^{\prime}}{\left(1+\lambda N+\bar{\lambda}^{\prime} N^{\prime}\right)^{2}}+4 s\right),
$$

mean trait and preference values converge to the equilibrium values $\bar{t}^{*}$ and $\bar{p}^{*}$ with

$$
\bar{t}^{*}=\frac{\frac{2 b d \bar{\lambda}^{\prime} N^{\prime}}{\left(1+\lambda N+\bar{\lambda}^{\prime} N^{\prime}\right)^{2}} \bar{t}^{\prime}+2 s t_{a}-a c_{R I} \frac{N^{\prime}}{N} \bar{t}^{\prime}}{\frac{2 b d \bar{\lambda}^{\prime} N^{\prime}}{\left(1+\lambda N+\bar{\lambda}^{\prime} N^{\prime}\right)^{2}}+2 s-a c_{R I} \frac{N^{\prime}}{N}},
$$

and

$$
\bar{p}^{*}=\bar{t}^{*}+c_{R I} \frac{N^{\prime}}{N}\left(\bar{t}^{*}-\bar{t}^{\prime}\right)
$$


These analytical expressions allow us to study the impact of the different evolutionary forces on the level of advergence of the trait displayed in the focal species toward the trait exhibited in the local mimicry ring $\left(\left|\bar{t}^{*}-\bar{t}^{\prime}\right|\right)$.

Otherwise when (14) is not verified the distances between mean trait and preference values in the focal and $\bar{t}^{\prime}$ become very large (not of order 1) (see Appendix 1.5).

$$
\left|\bar{t}^{*}-\bar{t}^{\prime}\right|=\frac{2 s}{\frac{2 b d \bar{\lambda}^{\prime} N^{\prime}}{\left(1+\lambda N+\bar{\lambda}^{\prime} N^{\prime}\right)^{2}}+2 s-a c_{R I} \frac{N^{\prime}}{N}}\left|t_{a}-\bar{t}^{\prime}\right| .
$$

Hence, if we assume no reproductive interference $\left(c_{R I}=0\right)$, we have $\left|\bar{t}^{*}-\bar{t}^{\prime}\right|<\left|t_{a}-\bar{t}^{\prime}\right|$, implying that the trait displayed in the focal species gets closer to the trait displayed in the local mimicry ring: we thus observe different evolutionary outcomes ranging from (a) mimicry to (b) imperfect mimicry, see Figures 1(a) and 1(b). The mimicry in the focal species becomes nearly perfect $\left(\left|\bar{t}^{*}-\bar{t}^{\prime}\right|\right.$ close to zero) when the strength of selection due to predation $\left(2 b d \bar{\lambda}^{\prime} N^{\prime} /\left(1+\lambda N+\bar{\lambda}^{\prime} N^{\prime}\right)^{2}\right)$ is large enough, as compared to the intra-specific constraints (s) (outcome (a) mimicry see Figure 1(a)).

However assuming reproductive interference between females from the focal species and males from the local mimicry ring impairs advergence. When reproductive interference is non null but has a limited strength, satisfying Inequality (14), Equation (17) implies that $\partial\left|\bar{t}^{*}-\bar{t}^{\prime}\right| / \partial c_{R I}>0$ (see Appendix 1.7.1). reproductive interference thus increases the gap between the traits displayed in the focal species and in the local mimicry ring, leading to imperfect mimicry in the focal species. Because females' preference generates sexual selection on male traits, reproductive interference promotes phenotypic divergence between the focal species and the local mimicry ring (see Equation (16)). Thus reproductive interference limits the advergence generated by Müllerian mimicry. 


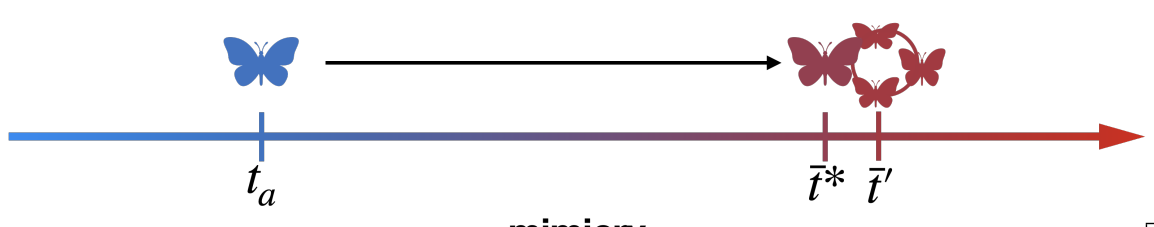

mimicry

(a)
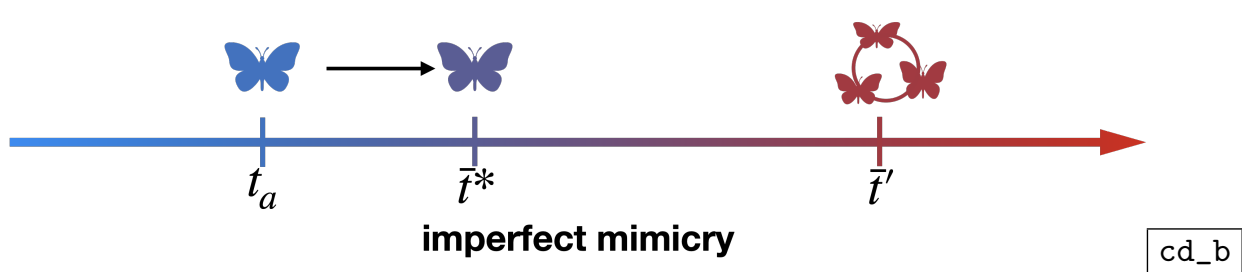

(b)

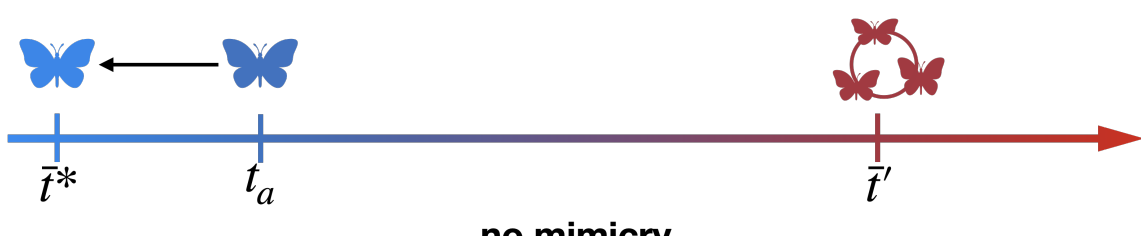

no mimicry

$c d \_c$

(c)

Figure 1: Illustration of three possible outcomes: (a) mimicry: the value of the trait in the focal species $\bar{t}$ becomes very close to the mean value displayed in the local mimicry ring $\bar{t}^{\prime},(\mathrm{b})$ imperfect mimicry: the value of the trait in the focal species $\bar{t}$ gets closer but stays distant from the mean value displayed in the local mimicry ring $\bar{t}^{\prime}$, (c) no mimicry: the value of the trait in the focal species $\bar{t}$ diverges away from the mean value displayed in the local mimicry ring $\bar{t}^{\prime}$.

However, when the cost associated with reproductive interference crosses a threshold, i.e. when

$$
\frac{N}{a N^{\prime}} \frac{4 b d \bar{\lambda}^{\prime} N^{\prime}}{\left(1+\lambda N+\bar{\lambda}^{\prime} N^{\prime}\right)^{2}} \leq c_{R I}<\frac{N}{a N^{\prime}}\left(\frac{4 b d \bar{\lambda}^{\prime} N^{\prime}}{\left(1+\lambda N+\bar{\lambda}^{\prime} N^{\prime}\right)^{2}}+4 s\right)
$$

then

$$
\left|\bar{t}^{*}-\bar{t}^{\prime}\right|>\left|t_{a}-\bar{t}^{\prime}\right|
$$

When assuming such an elevated cost of RI, imperfect mimicry is thus no longer observed, and reproductive interference rather promotes a strong divergence of the trait in the focal species (see 
Figure 1(c) for an illustration).

When inequality (14) is not satisfied, the phenotypic distance between the focal species and the local mimicry ring becomes very large. This very large divergence is biologically unrealistic but suggests that reproductive interference can promote phenotypic divergence between defended species living in sympatry. This unrealistic divergence stems from the weak female choosiness $(a=O(\varepsilon)$ ) assumed: since females have broad preference (because $a$ is low), females almost always accept heterospecific males, except when the difference between female preference and the trait displayed in the local mimicry ring is very high. Reproductive interference promotes female preference that limits fitness costs due to RI, and therefore promotes a large distance between female preference value and the value of the trait displayed in the local mimicry ring.

Relaxing the weak preference and predator discrimination hypothesis, i.e. assuming that $a=O(1)$ and $b=O(1)$, confirms that reproductive interference limits mimicry in the focal species. However, in this case, when a strong divergence is favored, this divergence becomes high but stays of order $O(1)$ (see Appendix 1.6). Indeed, as female choosiness is strong, this divergence strongly reduces fitness cost due to reproductive interference. Therefore stabilizing constraints on the trait in the focal species become more important than RI, thereby preventing very large divergence.

Strict female choosiness and moderate predator discrimination favor convergence of warning traits, despite reproductive interference.

The evolution of the trait in the focal species is shaped by two antagonistic evolutionary forces, generated by reproductive interference and Müllerian mimicry, respectively. Reproductive interference indirectly limits the advergence toward the local mimicry ring by impacting females' preference. Therefore, females' choosiness $a$ may be a key feature to understand the evolution of the trait in the focal species. The selection exerted by predation also depends on animal behavior through predators' learning. We thus investigate the impact of the strength of female choosiness $a$ and of predator discrimination coefficient $b$ on the evolution of the warning trait, allowing 
strong values of $a$ and $b$ (i.e. assuming $\left(H_{s}\right)$ ).

When the female choosiness is low, the selection promoting divergence generated by reproductive interference is limited. Indeed, females are not choosy and tend to accept all encountered males, including heterospecific males, whatever the direction of their preference. The difference in fitness cost due to reproductive interference between females with different preferences is then low, leading to poor selection due to reproductive interference. With a higher level of female choosiness, fitness cost due to reproductive interference depends more on the direction of preference, leading to higher selection caused by reproductive interference. Therefore, when the value of female choosiness $(a)$ increases from 0 to 1 , mimicry becomes less likely to evolve.

By contrast, Figure 2 highlights that mimicry becomes more likely as female choosiness $a$ takes values above 1 . Higher levels of female choosiness allow females to accurately distinguish males from their own species from individuals from the local mimicry ring. Accurate choice by females allows a quasi similarity of the traits of the focal species and of the local mimicry ring, without implying heterospecific mating, relaxing selection promoting divergence generated by reproductive interference. The evolutionary outcome is also impacted by selection exerted by predation. The predation rate depends on predators discrimination coefficient $b$, modulating their capacity to differentiate traits displayed in different mimetic species. When predators tend to generalize (when $b$ is approximately close to 0 ) there is no mimicry because there is no selection promoting similarity between the focal species and the local mimicry ring. When $b$ is greater than 0 but approximately similarly lower than 5, the higher discrimination of predators reduces the distances between the average traits displayed in the focal species and the local mimicry ring (Figure 2), leading to more accurate mimicry (see Appendix 1.7.2 for more detail). Surprisingly, when predator discrimination increases above a certain threshold, increased discrimination no longer promotes accurate mimicry (see sharp transition between mimicry (red) and no mimicry (blue) in Figure 2). When $b$ is high, individuals from the focal species benefit from protection gained by mimicry only when they look very similar to the individuals of the local mimicry ring. Reproductive interference makes such high similarity too costly, and therefore divergence of the 
trait in the focal species becomes promoted when predator discrimination is too high (Figure 2).

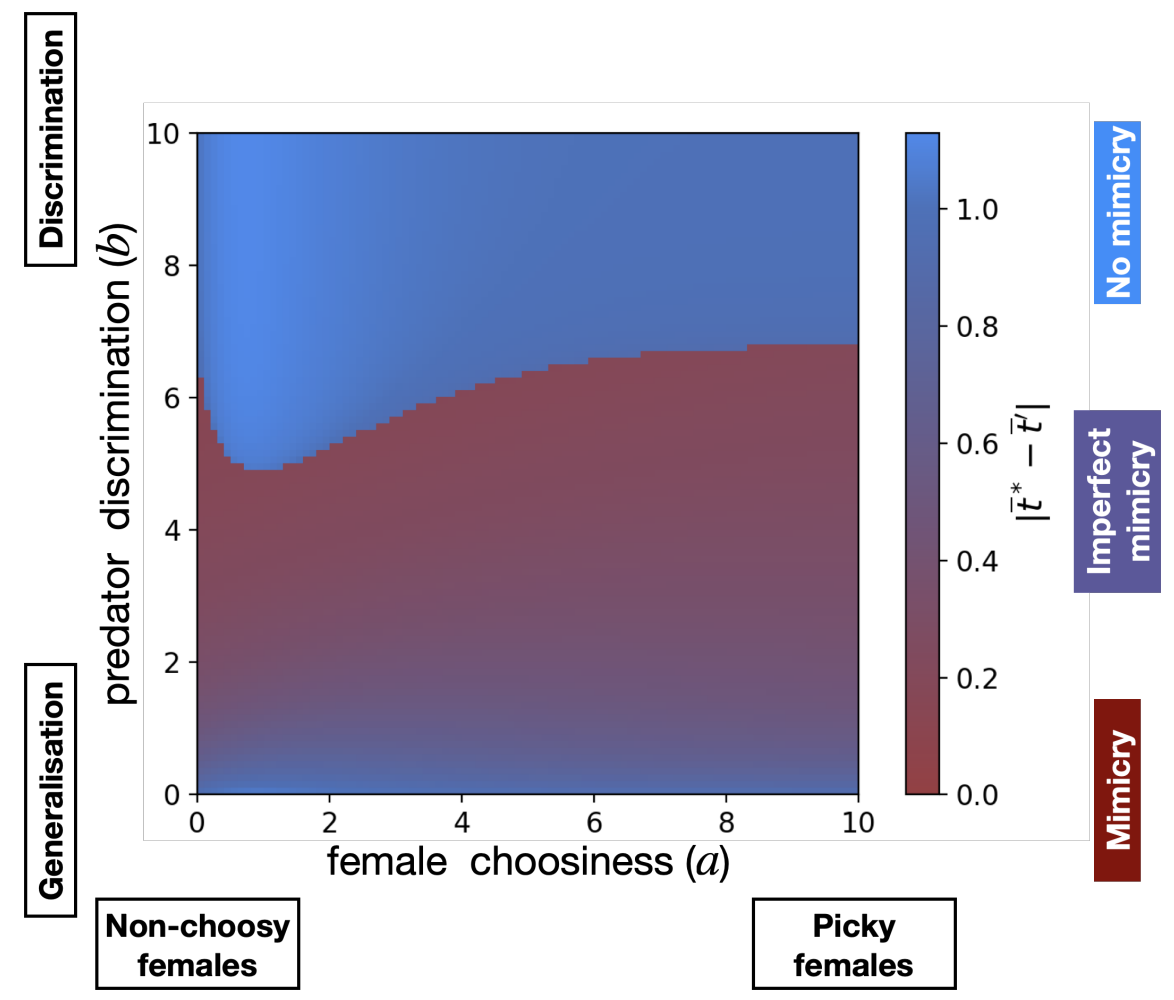

Figure 2: Influence of the predator discrimination $b$ and of the strength of female choosiness $a$ on the phenotypic distance between the focal species and the local mimicry ring. The plot reports values generated by numerical simulations performed using parameters values of the $\left(H_{s}\right)$ assumption. The parameter range generating mimicry, imperfect mimicry and no mimicry are shown in red, purple and blue respectively. We assume: $G_{t}=0.01, G_{p}=0.01, c_{R I}=0.002$, $d=0.02, \lambda=0.1, N=20, \bar{\lambda}^{\prime}=0.1, N^{\prime}=20, s=0.005, t_{a}=0, \bar{t}^{\prime}=1$.

Reproductive interference limits mimicry in species with low density.

The defense level of the focal species, i.e. $\lambda N$, is likely to impact the level of trait divergence. Without RI, $\partial\left|\bar{t}^{*}-\bar{t}^{\prime}\right| / \partial \lambda N>0$ indicates that the defense level in the focal species increases divergence from the trait displayed in the local mimicry ring. As expected, advergence toward the warning trait is thus more likely to happen when the focal species is weakly defended $(\lambda N$ 
small). Indeed, individuals from weakly defended species gain a limited protection from con-

specific individuals and have a great advantage gained from mimicry to the local community of well-defended species. By contrast, individuals of a well-defended species get protection from their conspecifics, and therefore the selective pressure favoring similarity to the local mimicry ring is weaker. In well-defended species, developmental and selective constraints promoting the ancestral trait $t_{a}$ exceed selection favoring similarity and promote the persistence of traits in the focal species distinct from the local mimicry ring. To understand the interaction between the two components of the defense level, we then compared the effect of the density $N$ and of the individual defense level $\lambda$ of the focal species, for different costs of choosiness (Figure 3). Without reproductive interference $\left(c_{R I}=0\right)$, yellow defense level $\lambda N$ isolines in Figure 3(a) show that the phenotypic distance between the focal species and the local mimicry ring only depends on the product $\lambda N$ (see). Moreover Figure 3(a) confirms that low defense level $\lambda N$ promotes mimicry in the focal species.

By contrast, assuming reproductive interference $\left(c_{R I}>0\right)$, Figures $3(\mathrm{~b})-(\mathrm{c})$ show that the evolutionary outcome depends more on the individual defense level than on the density of the focal species. The advergence toward the trait displayed in the local mimicry ring is observed when the individual defense level is low ( $\lambda$ approximately lower than 0.1 ) and when the density of the focal species is sufficiently large ( $N$ approximately greater than 5$)$. In this case, the defense level of the focal species $(\lambda N)$ is low, the protection gained by positive frequency-dependent selection within species is low, and the advergence toward the local mimicry ring is thus strongly promoted. Surprisingly, this advergence is impaired for similar values of defense level, when the density of the focal species is low ( $N$ approximately lower than 5 ). When the density of the focal species is low, females pay higher fitness costs due to RI, because they encounter more often heterospecific than conspecific males. Altogether, our results suggest that advergence of the warning traits is likely to happen for low level of individual defense in the focal species (i.e. Batesian $(\lambda=0)$ or quasi-Batesian ( $\lambda>0$ but small) mimicry) and when the density of the focal species is high. 


\section{Increased strength of reproductive interference $\left(c_{R I}\right)$}

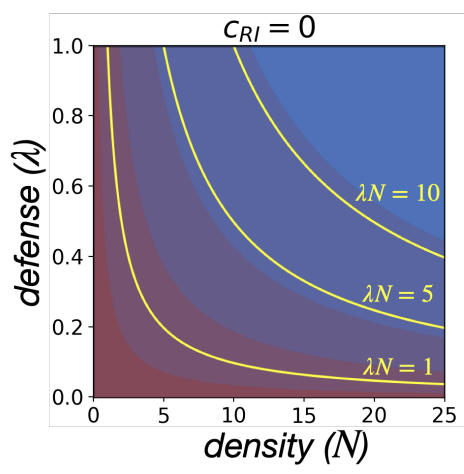

(a)

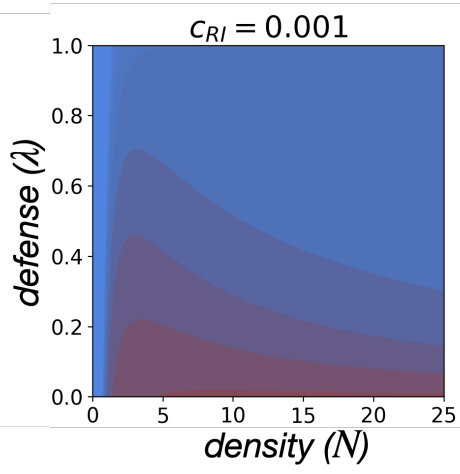

(b)

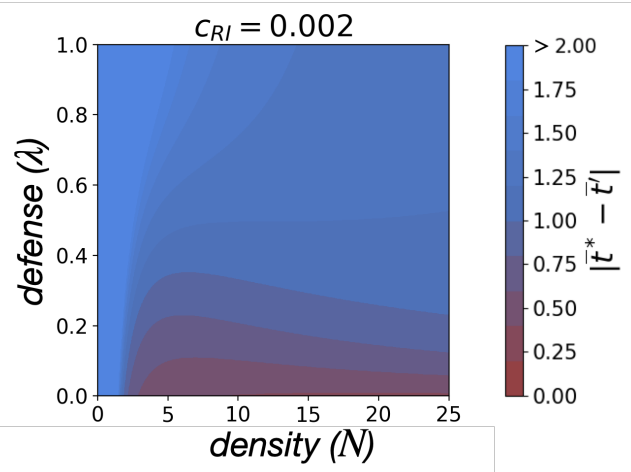

(c)

Figure 3: Influence of the density of the focal species $N$ and of individual defense level in the focal species $\lambda$ on the phenotypic distance between the focal species and the local mimicry ring, for different strengths of reproductive interference $c_{R I}$. The plot reports values generated by the analytic approximation assuming $\left(H_{w}\right)$. The parameter ranges generating mimicry, imperfect mimicry and no mimicry are shown in red, purple and blue, respectively. Phenotypic distances greater than 2 are shown in blue. Yellow lines indicate equal levels of defense level in the focal species $\lambda N$. Different values of strengths of reproductive interference are assumed: (a) $c_{R I}=0$, (b) $c_{R I}=0.001$, (c) $c_{R I}=0.002$. We assume: $a=0.01, d=0.05, b=0.01, \bar{\lambda}^{\prime}=0.1, N^{\prime}=20$, $s=0.00005, t_{a}=0, \bar{t}^{\prime}=1$.

\section{Evolution of warning traits within two interacting species}

\section{Method}

Because reproductive interference may impact the direction of advergence, and because ancestral traits that might differ among species may influence the evolution of traits, we build an extension of the previous model by assuming two sympatric species, whose traits can evolve. This twospecies model allows investigating the eco-evolutionary dynamics of trait convergence. We thus follow the evolution of traits ( $t_{1}$ and $t_{2}$ in species 1 and 2 , respectively) and female choosiness ( $p_{1}$ 
and $p_{2}$ in species 1 and 2, respectively). Because the two species are assumed to be fully sym-

patric, individuals from both species face the same community of predators, and can encounter each others. The evolution of trait and preference in both species are thus shaped by both (1) Müllerian mimicry and (2) reproductive interference caused by heterospecific mating.

Assuming that traits and preferences have an autosomal polygenic basis with additive effects in both species, the change in the mean values of traits $\left(\bar{t}_{1}, \bar{t}_{2}\right)$ and preferences $\left(\bar{p}_{1}, \bar{p}_{2}\right)$ can be approximated using:

$$
\left(\begin{array}{c}
\Delta \bar{t}_{i} \\
\Delta \bar{p}_{i}
\end{array}\right)=\frac{1}{2}\left(\begin{array}{cc}
G_{t_{i}} & C_{t_{i} p_{i}} \\
C_{t_{i} p_{i}} & G_{p_{i}}
\end{array}\right)\left(\begin{array}{l}
\beta_{t_{i}} \\
\beta_{p_{i}}
\end{array}\right) \approx \frac{1}{2}\left(\begin{array}{c}
G_{t_{i}} \beta_{t_{i}} \\
G_{p_{i}} \beta_{p_{i}}
\end{array}\right),
$$

where for $i \in\{1,2\}$ the variables $\beta_{t_{i}}$ and $\beta_{p_{i}}$ describe the selective forces acting on the traits and preferences $t_{i}$ and $p_{i}$ (defined in Appendix 2.2). $G_{t_{i}}$ and $G_{p_{i}}$ are the additive genetic variances of $t_{i}$ and $p_{i}$ and $C_{t_{i}} p_{i}$ is the additive genetic covariance between $t_{i}$ and $p_{i}$. As in the previously described model, following (Iwasa et al., 1991), we assume that $G_{t_{i}}$ and $G_{p_{i}}$ are positive constants maintained by an equilibrium between selection and recurrent mutations. As we already showed (see Appendix 1.3), the additive genetic covariance $C_{t_{i} p_{i}}$ is small in comparison with $G_{t_{i}}$ and $G_{p_{i}}$, allowing us to approximate the change in the mean values of trait and preference as done in (20).

Female choosiness $\left(a_{1}\right.$ and $\left.a_{2}\right)$, strength of selective and developmental constraints ( $s_{1}$ and $\left.s_{2}\right)$, ancestral trait $\left(t_{a 1}\right.$ and $\left.t_{a 2}\right)$, defense level $\left(\lambda_{1}\right.$ and $\left.\lambda_{2}\right)$ carried out by individuals, as well as density $\left(N_{1}\right.$ and $\left.N_{2}\right)$ can differ between species 1 and 2 . All variables and parameters used in the two-population model are summed up in the supplementary Table A1.

\section{Model exploration.}

To specifically test the effect of species interactions on trait convergence, we assume the two species to be initially isolated and explore trait evolution when they become sympatric. We also assume the initial mean female preferences value to equal ancestral trait values within each species. The dynamics of the mean trait and preference values when both species become in contact are then given by Equation (20). 
Exploration assuming weak female choosiness $\left(a_{1}=O(\varepsilon)\right.$ and $a_{2}=O(\varepsilon)$ ) and predator discrimination $\left(b=O(\varepsilon)\right.$ ) (Hypothesis $\left(H_{w}\right)$ ). When we assume that $a_{1}, a_{2}$ and $b$ are of order $\varepsilon$ and $s$ is of order $\varepsilon^{2}$, we can analytically determine the mean traits and preferences values at equilibrium in most cases. In the few remaining cases, we use numerical analyses to estimate these values (see Appendix 2.4). We note $\bar{t}_{1}^{*}, \bar{t}_{2}^{*}, \bar{p}_{1}^{*}$ and $\bar{p}_{2}^{*}$ the mean traits and preferences at equilibrium. We follow the quantity $\left|\bar{t}_{1}^{*}-\bar{t}_{2}^{*}\right|$, i.e. the mean phenotypic distance between both species at equilibrium. To determine the direction of advergence, we check whether the trait values observed at equilibrium (i.e. $\bar{t}_{1}^{*}$ and $\left.\bar{t}_{2}^{*}\right)$ are closer to the ancestral trait value of species 1 or of species $2\left(t_{a 1}\right.$ or $t_{a 2}$ respectively).

Exploration assuming strong female choosiness $\left(a_{1}=O(1)\right.$ and $\left.a_{2}=O(\varepsilon)\right)$ and predator discrimination $(b=O(1))$ (Hypothesis $\left(\left(H_{s}\right)\right)$ ). Similarly to the previous model exploration, we use numerical simulations to study cases where female choosiness and predator discrimination are strong $\left(a_{1}=\right.$ $O(1), a_{2}=O(1), b=O(1)$ and $\left.s=O(\varepsilon)\right)$.

As in the exploration of the previous model, by default we report results assuming $\left(H_{w}\right)$ and check the validity of these results when assuming $\left(H_{S}\right)$ (see Appendix 2.5).

\section{Reproductive interference may reverse the direction of advergence.}

As previously unraveled, reproductive interference may limit the convergence of warning traits, depending on the level of defense and on the density of the two mimetic species (Figure 3(c)). By studying the two-species model, we can also investigate how reproductive interference may influence the direction of advergence, depending on the relative levels of defense of the two species, generated by their densities and individual strength of defense $\left(\lambda_{1} N_{1}\right.$ and $\lambda_{2} N_{2}$, respectively). The fitness costs caused by reproductive interference then also differ between the two species, depending on their relative abundances, leading to unbalanced selective pressures on trait evolution in the two species: females from the rarest species suffer more from RI, because 
they encounter heterospecific more often than conspecific males.

To investigate how the relative defense levels of the two species affect the direction of ad-

vergence, we study the phenotype at equilibrium in both species, for different values of the two components of the defense level of species 1: the individual defense level $\left(\lambda_{1}\right)$ and the density $\left(N_{1}\right)$. Here we assumed that species 2 is already well protected $\left(\lambda_{2}=0.1, N_{2}=10\right)$.

When assuming no reproductive interference $\left(c_{R I}=0\right)$, species traits converge toward the ancestral trait $t_{a}$ initially displayed in the best defended species. In Figures $4(\mathrm{a})$ and $4(\mathrm{~b})$, individuals from the poorly defended species 1 (i.e. when $\lambda_{1} N_{1}$ is low) get weak protection from conspecific individuals and thus have a greater advantage to look similar to individuals of species 2 . The more species 1 is defended, i.e. the greater $\lambda_{1} N_{1}$ is, the closer its mean trait value is to the ancestral trait value $t_{a 1}$ (see Figure 4(a)). Such increase in the defense level of species 1 also impacts the evolution of trait in the sympatric species 2 (see Figure 4(b)): when the individual defense level in species $1\left(\lambda_{1}\right)$ is below a threshold, the more individuals from species 1 are protected, the more the mean trait value in species 2 moves away from its ancestral trait $\left(t_{a 2}\right)$. Surprisingly, above this threshold, the better protected species 1 is, the closer the mean trait value in species 2 gets to its ancestral trait value $\left(t_{a 2}\right)$. The weak discrimination capacities of predators assumed favors such evolution of divergent traits. Individuals from species 2 may thus gain some protection from species 1 even with a poor resemblance. When species 1 is highly protected (high $\lambda_{1} N_{1}$ ), the level of protection gained by individuals of species 2 because of their resemblance with individuals of species $1\left(\lambda_{1} N_{1} \exp \left[-b\left(\bar{t}_{1}-\bar{t}_{2}\right)^{2}\right]\right)$ is high, even when individuals of species 2 display poorly mimetic trait, close to the ancestral trait value $\left(\bar{t}_{2}=t_{a 2}\right)$. The selective and developmental constraints within species 2 then keep the trait in species 2 close to its ancestral trait value $t_{a 2}$.

When assuming positive strength of reproductive interference $\left(c_{R I}>0\right)$, the trait value of species 1 almost never converges toward the trait value initially displayed in species $2\left(t_{a 2}\right)$. On the contrary, individuals of species 2 can mimic individuals of species 1 (see blue zone in Figure 4(d)), when the defense level of individuals of species 1 is high and when species 1 is rare. Because individuals from both species are well defended (high $\lambda_{1}$ and $\lambda_{2}$ ), individuals of both 


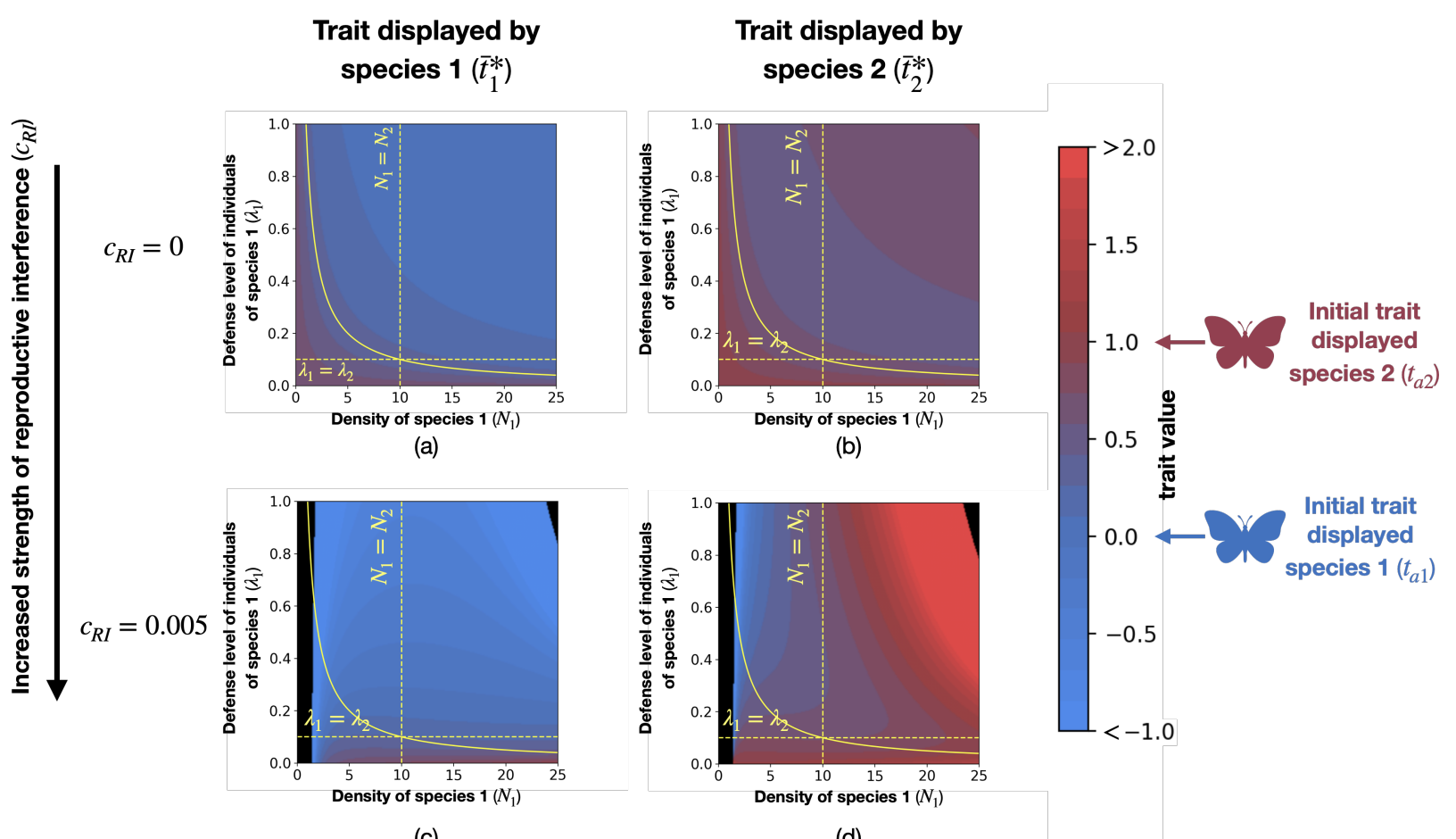

(c)

(d)

Figure 4: Influence of the density and of the individual defense level in species 1 ( $N_{1}$ and $\lambda_{1}$ ) on the traits displayed in both species, for different strengths of reproductive interference $\left(c_{R I}\right)$. Plots report values obtained with the analytic approximation, assuming $\left(H_{w}\right)$. Mean trait values can become very large (black zone). Trait values greater than 2 (resp. lower than -1 ) are shown in red (resp. blue). The yellow solid line shows the case where both species have the same level of defense $\left(\lambda_{1} N_{1}=\lambda_{2} N_{2}\right)$. Below (resp. above) this line species 1 has a lower (resp. higher) level of defense than species 2. Different values of strengths of reproductive interference are assumed: (a) and (b) $c_{R I}=0,(\mathrm{c})$ and (d) $c_{R I}=0.005$. We assume: $a_{1}=a_{2}=0.01, d=0.05$, $b=0.01, \lambda_{2}=0.1, N_{2}=10, s_{1}=s_{2}=0.000005, t_{a 1}=0, t_{a 2}=1$.

species benefit from looking similar. However, when species 1 is rarer, this leads to an increased cost of RI in species 1, inhibiting convergence towards the ancestral trait value displayed in the alternative species $\left(t_{a 2}\right)$ (see Figure $4(\mathrm{c})$ ). Since predation pressure promotes convergence of traits of both species, the mean trait in species 2 becomes closer to species 1 ancestral trait value $t_{a 1}$. Surprisingly, such advergence also happens when individuals of species 2 are more 
defended than individuals from species 1, i.e. $\lambda_{2} N_{2}>\lambda_{1} N_{1}$ (see blue zones in Figures 4(c) and (d) below the yellow solid line). By contrast, when the density in species 1 exceeds the density in species 2, individuals from both species exhibit traits close to their ancestral traits. As previously explained, both species are well-protected and then gain little from mimicry. However, the phenotypic distance between the two species is higher than in the previous case, because reproductive interference further promotes trait dissimilarity (see Figure 4).

Our results highlight that reproductive interference impacts the evolution of warning traits, and may even reverse the direction of advergence, depending on the relative abundances and defense levels of sympatric species.

Ancestral trait values in interacting species strongly modulate phenotypic divergence driven by reproductive interference.

Our previous results highlight that reproductive interference limits the convergence of warning traits. This effect is also observed in the model investigating the evolution of traits in two interacting species. However, the effect of reproductive interference on trait divergence strongly depends on the ancestral trait values initially displayed in the two species in the absence of ecological interactions $\left(t_{a 1}\right.$ and $\left.t_{a 2}\right)$. When these ancestral trait values differ, an increase in strength of reproductive interference leads to a progressive increase in the phenotypic distance between both species (see Figure 5).

Surprisingly, when the ancestral trait values are the same in both species $\left(t_{a 1}=t_{a 2}\right)$, an increase in the strength of reproductive interference $\left(c_{R I}\right)$ has no effect on the phenotypic distance between both species, as long as this cost of reproductive interference remains below a threshold (see Figures 6(a) and 6(b)). This effect is observed assuming weak $\left(H_{w}\right)$ or strong $\left(H_{s}\right)$ female choosiness and predator discrimination.

However, when the strength of reproductive interference $\left(c_{R I}\right)$ is greater than this threshold, assuming weak female choosiness and predator discrimination $\left(H_{w}\right)$, the phenotypic distance between both species becomes instantaneously very large, while their initially displayed ancestral 


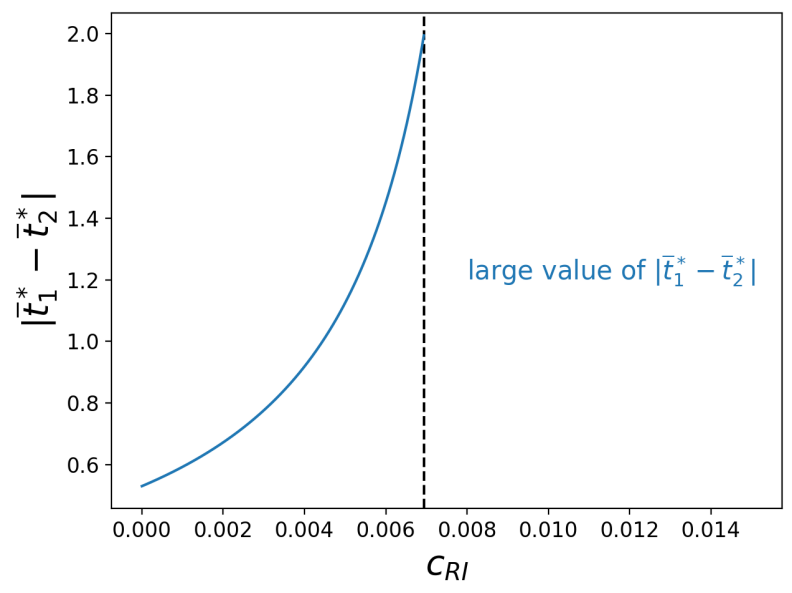

Figure 5: Influence of the strength of reproductive interference $c_{R I}$ on the phenotypic distances between the two species, when they have different ancestral traits $\left(t_{a 1} \neq t_{a 2}\right)$. The plot reports values obtained by the analytic approximation assuming $\left(H_{w}\right)$. We assume: $a_{1}=a_{2}=0.01$, $d=0.02, b=0.01, \lambda_{1}=\lambda_{2}=0.1, N_{1}=N_{2}=10, s_{1}=s_{2}=0.000005, t_{a 1}=0, t_{a 2}=1$.

trait values $t_{a 1}$ and $t_{a 2}$ are equal. A similar trend is observed when female choosiness and predator discrimination are strong $\left(H_{s}\right)$ : the phenotypic distance is null when the strength of reproductive interference remains below a threshold, but it quickly increases to a high value when the strength of reproductive interference crosses the threshold (see Figure 6(b)).

Our results highlight that the ancestral trait values strongly modulate the effect of reproductive interference on the convergence of warning traits. Surprisingly, drastic divergence might be promoted by a strong cost of RI, even when the optimal phenotypes are the same in the two interacting species.

\section{Discussion}

\section{Reproductive interference leads to unexpected evolution of warning trait}

Our model shows that reproductive interference limits the convergence of warning traits in defended species living in sympatry. The effect of reproductive interference strongly depends on 


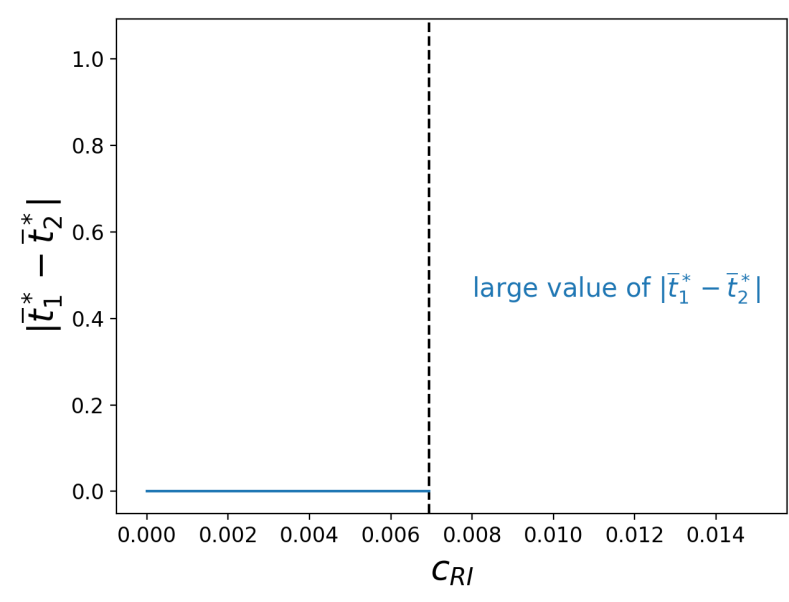

(a)

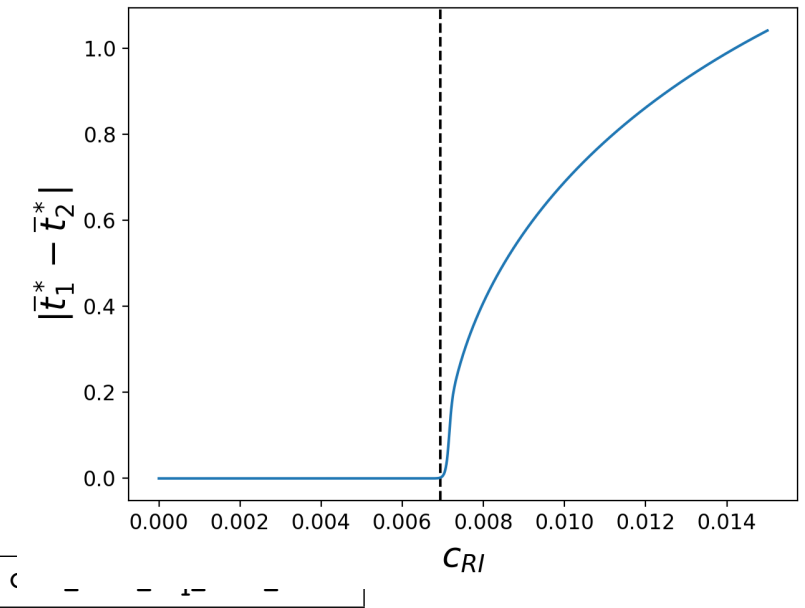

(b)

Figure 6: Influence of the strength of reproductive interference $c_{R I}$ on the phenotypic distances between the two species when they share the same ancestral traits interaction $\left(t_{a 1}=t_{a 2}\right)$, assuming (a) $\left(H_{w}\right)$ weak or (b) $\left(H_{s}\right)$ strong female choosiness and predator discrimination. Plots report values obtained by (a) the analytic approximation assuming $\left(H_{w}\right)$ and (b) numerical simulations to use parameters values corresponding to $\left(H_{s}\right)$ assumption. Different values of female choosiness, predator discrimination coefficient and strength of selective and developmental constraints are assumed : (a) $a=0.01, b=0.01$ and $s=0.00005$, (b) $a=1, b=1$ and $s=0.005$. We assume: $G_{t}=0.01, G_{p}=0.01, d=0.02, \lambda_{1}=\lambda_{2}=0.1, N_{1}=N_{2}=10, t_{a 1}=t_{a 2}=1$.

the relative abundances of interacting species, leading to surprising evolutionary outcomes. For example, in rare defended species, selection favoring mimicry towards the local community of defended species is expected to be strong. Nevertheless, our model shows that the elevated costs of reproductive interference prevents the evolution of mimicry in the rarest species, because females then encounter much more heterospecific than conspecific males.

Our results thus shed some light not only on the persistence of distinct warning traits within local communities of defended species in the wild, but also on the emergence of these distinct warning traits in the first place. Mimetic diversity is a apparent paradox but several hypotheses have been suggested to promote the persistence of different warning signals, including for 
instance the segregation of predators within microhabitats (Beccaloni, 2008; Devries et al., 1999;

Elias et al., 2008; Willmott et al., 2017). The spread of distinct warning traits has then frequently been shown to be promoted by demographic stochasticity, as in shifting balance models (Mallet and Joron, 1999; Sherratt, 2006) or in other models combining predator behaviours, such as neophobia, to stochastic effects (Aubier and Sherratt, 2015). However, these models do not provide any selective mechanism explaining the emergence of warning signal with different levels of divergence, contributing to mimetic diversity. By contrast, reproductive interference selects for different levels of divergence in warning traits, and could be a major driver of the diversity of mimetic traits. Note that other mechanisms may generated gradual departure from the ancestral trait value and may also contribute to the diversity of mimetic traits: the evolution of aposematic signals in defended species away from those exhibited in Batesian mimics has been theoretically shown to be promoted (Franks et al., 2009). Artificial modification of the warning trait of mated females has also been demonstrated to reduce harassment by males in the butterfly H. erato, and would therefore allow them to lay more eggs, suggesting that evolution of slightly divergent trait could be promoted in females (Merrill et al., 2018).

Reproductive interference may particularly promote the emergence and persistence of a distinct warning trait in low-density populations of warning species coming in contact with a local mimicry ring that exhibits a different warning trait. Nevertheless, our model does not take into account the dynamics of population density, and therefore ignores the extinction risk of lowdensity populations. Such non-mimetic populations with low density might nevertheless persist in the wild, when the level of individual defense is sufficiently high.

Our model also brings new insights to the ecological processes driving the direction of advergence in warning traits. In the absence of RI, warning traits are expected to evolve in the less defended species (e.g. low density populations and/or low level of individual defense) and become mimetic of better-defended species living in sympatry (Balogh and Leimar, 2005; Franks and Sherratt, 2007). When considering the costs of reproductive interference, however, this general trend no longer holds, because warning traits in the most abundant species can evolve toward 
the warning trait of the less abundant one.

\section{The evolution of imperfect mimicry}

Our results suggest that reproductive interference alone is unlikely to promote imperfect mimicry, in contradiction with previous predictions (Pfennig and Kikuchi, 2012). When sympatric species share the same ancestral trait, we indeed observe either perfect mimicry or strong trait divergence, depending on the strength of reproductive interference. In our model, imperfect mimicry is observed only when interacting species have different ancestral traits. The contrasted developmental and selective constraints underwent by the different species may thus play an important role in imperfect mimicry. These different constraints are likely strongly correlated with the phylogenetic distances between species: closely-related species are likely to share same genetic basis and developmental pathway leading to the warning trait and to also share similar environments, due to niche conservatism (Chazot et al., 2014; Elias et al., 2008; Joshi et al., 2017). Our results suggest that imperfect mimicry could not be promoted among closely-related species experiencing high levels of reproductive interference but limited differences in ancestral traits. Imperfect mimicry may rather be observed between phylogenetically distant species, subject to more strikingly different developmental and selective constraints, where reproductive interference might be more limited. Distantly related species indeed might have diverged in other traits, facilitating mate recognition through different cues. 


\section{How important are developmental and selective constraints in the evolution of}

\section{warning trait?}

Estimating the level of developmental and selective constraints potentially shaping the evolution of warning traits is not straightforward. Genetic studies, reviewed in Joron et al. (2006), show that

\section{Evolution of female preferences limiting the reproductive interference costs}

\section{generated by mimicry}

Our results show the evolution of close similarity between species, despite the costs of reproductive interference. When female choosiness is high, successful species recognition might occur without decreasing the protection brought by mimicry. Such situation arises when predators largely generalize, and therefore do not discriminate imperfect mimics. Such differences in the discrimination of color patterns between prey and predators are frequently observed in the wild. 
For instance, Llaurens et al. (2014) showed that the variations in color pattern between co-mimetic

species from the distantly related genus Heliconius and Melinaea might be better perceived by the Heliconius butterflies themselves rather than by avian predators. The evolution of visual perception in females could also enhance species discrimination without impairing mimicry. The evolution of vision in females from the Heliconius butterflies indeed coincides with the evolution of the yellow pigments 3-OH-kinurenin displayed on their wings (Bybee et al., 2012). The evolution of high discrimination capacities in mimetic prey, as well as the evolution of mating cue undetected by predators could thus limit the cost of reproductive interference in mimetic prey. In butterflies, mate choice indeed often relies on pheromones that may strongly differ among closely-related mimetic species (Darragh et al., 2017; González-Rojas et al., 2020). Micro-habitat differences among mimetic species may also allow reducing heterospecific encounters while still enjoying the benefit of mimicry by sharing the same predator community (Estrada and Jiggins, 2002). In three Morpho butterfly species displaying local convergence in wing patterns (Llaurens et al., 2021), temporal segregation in patrolling activity has been observed between species sharing similar color patterns (Le Roy et al., 2020), which may strongly limit heterospecific rivalry.

The levels of reproductive interference among mimetic species might thus be modulated by the evolution of the converging traits themselves, as well as the evolution of other traits involved in species interactions.

\section{Conclusion}

Our analytical and numerical results highlight the role of reproductive interference in the evolution of warning traits. They confirm that reproductive interference limits the convergence of warning trait promoted by mutualistic interactions. Surprisingly, reproductive interference can change the direction of the advergence of warning trait when the species densities are unbalanced. Our results also suggest that reproductive interference alone cannot explain imperfect mimicry, highlighting the role of ancestral traits as well as developmental and selective con- 
straints in the evolution of imperfect mimicry.

\section{Data Availability}

Codes are available online: github.com/Ludovic-Maisonneuve/limits-of-ev-conv

\section{Acknowledgments}

LM would like to thank Dorian Ni for feedbacks on the mathematical part of the study. LM and VL would like to thank Charline Pinna and the whole 'Evolution and Development of Phenotypic Variations' team for stimulating discussions on the evolution of the warning traits. The authors would like to thank the ANR SUPERGENE (ANR-18-CE02-0019) for funding the PhD of LM, and the Emergence program from Paris city council for supporting the team of VL. This work was also supported by the Chair "Modélisation Mathématique et Biodiversité" of VEOLIA- Ecole Polytechnique-MNHN-F.X.

\section{Conflict of interest disclosure}

The authors of this preprint declare that they have no conflict of interest with the content of this article.

\section{References}

Arias, M., le Poul, Y., Chouteau, M., Boisseau, R., Rosser, N., Théry, M., and Llaurens, V. (2016). Crossing fitness valleys: empirical estimation of a fitness landscape associated with polymorphic mimicry. Proceedings of the Royal Society B: Biological Sciences, 283(1829):20160391.

Aubier, T. G. and Sherratt, T. N. (2015). Diversity in müllerian mimicry: The optimal predator sampling strategy explains both local and regional polymorphism in prey. Evolution, 69(11):2831-2845. 
Balogh, A. C. and Leimar, O. (2005). Müllerian mimicry: an examination of fisher's theory of gradual evolutionary change. Proceedings of the Royal Society B: Biological Sciences, 272(1578):2269-2275.

Barton, N. H. and Turelli, M. (1991). Natural and sexual selection on many loci. Genetics, 127(1):229-255.

Beccaloni, G. (1997). Ecology, natural history and behaviour of ithomiine butterflies and their mimics in ecuador (lepidoptera: Nymphalidae: Ithomiinae). Tropical Lepidoptera, 8:103-124.

Beccaloni, G. W. (2008). Vertical stratification of ithomiine butterfly (Nymphalidae: Ithomiinae) mimicry complexes: the relationship between adult flight height and larval host-plant height. Biological Journal of the Linnean Society, 62(3):313-341.

Benitez-Vieyra, S., de Ibarra, N. H., Wertlen, A. M., and Cocucci, A. A. (2007). How to look like a mallow: evidence of floral mimicry between turneraceae and malvaceae. Proceedings of the Royal Society B: Biological Sciences, 274(1623):2239-2248.

Benson, W. W. (1972). Natural selection for mullerian mimicry in heliconius erato in costa rica. Science, 176(4037):936-939.

Boussens-Dumon, G. and Llaurens, V. (2021). Sex, competition and mimicry: an eco-evolutionary model reveals how ecological interactions shape the evolution of phenotypes in sympatry. Oikos, in press.

Briolat, E. S., Burdfield-Steel, E. R., Paul, S. C., Rönkä, K. H., Seymoure, B. M., Stankowich, T., and Stuckert, A. M. M. (2019). Diversity in warning coloration: selective paradox or the norm? Biological Reviews, 94(2):388-414. 
allow a color signal to serve both mimicry and intraspecific communication. The American Naturalist, 179(1):38-51. PMID: 22173459.

Chazot, N., Willmott, K. R., Santacruz Endara, P. G., Toporov, A., Hill, R. I., Jiggins, C. D., and Elias, M. (2014). Mutualistic mimicry and filtering by altitude shape the structure of andean butterfly communities. The American Naturalist, 183(1):26-39. PMID: 24334733.

Chouteau, M., Arias, M., and Joron, M. (2016). Warning signals are under positive frequencydependent selection in nature. Proceedings of the National Academy of Sciences, 113(8):21642169.

Cortesi, F. and Cheney, K. L. (2010). Conspicuousness is correlated with toxicity in marine opisthobranchs. Journal of Evolutionary Biology, 23(7):1509-1518.

Dalziell, A. H. and Welbergen, J. A. (2016). Mimicry for all modalities. Ecology Letters, 19(6):609_ 619.

Darragh, K., Vanjari, S., Mann, F., Gonzalez-Rojas, M. F., Morrison, C. R., Salazar, C., Pardo-Diaz, C., Merrill, R. M., McMillan, W. O., Schulz, S., and Jiggins, C. D. (2017). Male sex pheromone components in Heliconius butterflies released by the androconia affect female choice. Peer], 5:e3953.

Devries, P., LANDE, R., and Murray, D. (1999). Associations of co-mimetic ithomiine butterflies on small spatial and temporal scales in a neotropical rainforest. Biological Journal of the Linnean Society, 67:73-85.

Elias, M., Gompert, Z., Jiggins, C., and Willmott, K. (2008). Mutualistic interactions drive ecological niche convergence in a diverse butterfly community. PLOS Biology, 6(12):1-1.

Estrada, C. and Jiggins, C. (2002). Patterns of pollen feeding and habitat preference among heliconius species. Ecological Entomology, 27:448 - 456. 
Estrada, C. and Jiggins, C. D. (2008). Interspecific sexual attraction because of convergence in warning colouration: is there a conflict between natural and sexual selection in mimetic species? Journal of Evolutionary Biology, 21(3):749-760.

Flanagan, N. S., Tobler, A., Davison, A., Pybus, O. G., Kapan, D. D., Planas, S., Linares, M., Heckel, D., and McMillan, W. O. (2004). Historical demography of müllerian mimicry in the neotropical heliconius butterflies. Proceedings of the National Academy of Sciences, 101(26):9704-9709.

Franks, D. W. and Noble, J. (2004). Batesian mimics influence mimicry ring evolution. Proceedings of the Royal Society of London. Series B: Biological Sciences, 271(1535):191-196.

Franks, D. W., Ruxton, G. D., and Sherratt, T. N. (2009). Warning signals evolve to disengage batesian mimics. Evolution, 63(1):256-267.

Franks, D. W. and Sherratt, T. N. (2007). The evolution of multicomponent mimicry. Journal of Theoretical Biology, 244(4):631 - 639 .

González-Rojas, M. F., Darragh, K., Robles, J., Linares, M., Schulz, S., McMillan, W. O., Jiggins, C. D., Pardo-Diaz, C., and Salazar, C. (2020). Chemical signals act as the main reproductive barrier between sister and mimetic $<$ i $>$ heliconius $</$ i $>$ butterflies. Proceedings of the Royal Society B: Biological Sciences, 287(1926):20200587.

Goodale, E. and Kotagama, S. W. (2006). Vocal mimicry by a passerine bird attracts other species involved in mixed-species flocks. Animal Behaviour, 72(2):471 - 477.

Grill, C. P. and Moore, A. (1998). Effects of a larval antipredator response and larval diet on adult phenotype in an aposematic ladybird beetle. Oecologia, 114:274-282.

Gröning, J. and Hochkirch, A. (2008). Reproductive interference between animal species. The Quarterly Review of Biology, 83(3):257-282. PMID: 18792662. 
Hines, H. M., Counterman, B. A., Papa, R., Albuquerque de Moura, P., Cardoso, M. Z., Linares, M., Mallet, J., Reed, R. D., Jiggins, C. D., Kronforst, M. R., and McMillan, W. O. (2011). Wing patterning gene redefines the mimetic history of heliconius butterflies. Proceedings of the National Academy of Sciences, 108(49):19666-19671.

Iwasa, Y., Pomiankowski, A., and Nee, S. (1991). The evolution of costly mate preferences ii. the “handicap" principle. Evolution, 45(6):1431-1442.

Jiggins, C. D., Naisbit, R. E., Coe, R. L., and Mallet, J. (2001). Reproductive isolation caused by colour pattern mimicry. Nature, 411(6835):302-305.

Joron, M. and Iwasa, Y. (2005). The evolution of a müllerian mimic in a spatially distributed community. Journal of Theoretical Biology, 237(1):87 - 103. an evo-devo model for understanding phenotypic diversity. Heredity, 97(3):157-167.

Joshi, J., Prakash, A., and Kunte, K. (2017). Evolutionary assembly of communities in butterfly mimicry rings. The American Naturalist, 189(4):E58-E76.

Kapan, D. D. (2001). Three-butterfly system provides a field test of müllerian mimicry. Nature, 409(6818):338-340.

Kirkpatrick, M., Johnson, T., and Barton, N. (2002). General models of multilocus evolution. Genetics, 161(4):1727-1750.

Kronforst, M. R., Young, L. G., Kapan, D. D., McNeely, C., O’Neill, R. J., and Gilbert, L. E. (2006). Linkage of butterfly mate preference and wing color preference cue at the genomic location of wingless. Proceedings of the National Academy of Sciences, 103(17):6575-6580.

Kyogoku, D. (2015). Reproductive interference: ecological and evolutionary consequences of interspecific promiscuity. Population Ecology, 57(2):253-260. 
Le Roy, C., Roux, C., Authier, E., Bastide, H., Debat, V., and Llaurens, V. (2020). Convergent morphology and divergent phenology: unravelling the coexistence of mimetic morpho butterfly species. bioRxiv.

Lev-Yadun, S. (2009). Müllerian mimicry in aposematic spiny plants. Plant Signaling \& Behavior, 4(6):482-483. PMID: 19816137.

itans:arns2013

McPeek, M. A. and Gavrilets, S. (2006). The evolution of female mating preferences: Differentiation from species with promiscious males can promotes speciation. Evolution, 60(10):1967 1980. 
Merrill, R. M., Chia, A., and Nadeau, N. J. (2014). Divergent warning patterns contribute to assortative mating between incipient heliconius species. Ecology and Evolution, 4(7):911-917. manipulation of heliconius warning patterns reduces harassment of previously mated females. bioRxiv.

Mérot, C., Frérot, B., Leppik, E., and Joron, M. (2015). Beyond magic traits: Multimodal mating cues in heliconius butterflies. Evolution, 69(11):2891-2904.

Nagylaki, T. (1993). The evolution of multilocus systems under weak selection. Genetics, 134(2):627-647.

Naisbit, R. E., Jiggins, C. D., and Mallet, J. (2001). Disruptive sexual selection against hybrids contributes to speciation between Heliconius cydno and Heliconius melpomene. Proceedings of the Royal Society of London. Series B: Biological Sciences, 268(1478):1849-1854.

Ojala, K., Lindsröm, L., and Mappes, J. (2007). Life-history constraints and warning signal expression in an arctiid moth. Functional Ecology, 21(6):1162-1167.

Pfennig, D. W. and Kikuchi, D. W. (2012). Competition and the evolution of imperfect mimicry. Current Zoology, 58(4):608-619.

Pomiankowski, A. and Iwasa, Y. (1993). Evolution of multiple sexual preferences by fisher's runaway process of sexual selection. Proceedings: Biological Sciences, 253(1337):173-181.

Rice, S. H. (2004). Evolutionary theory: mathematical and conceptual foundations. Sinauer Associates, Sunderland, Mass., USA. 62(11):2913-2921. 
Sanders, K., Malhotra, A., and Thorpe, R. (2006). Evidence for a müllerian mimetic radiation in asian pitvipers. Proceedings of the Royal Society B: Biological Sciences, 273(1590):1135-1141.

Savage, J. M. and Slowinski, J. B. (1992). The colouration of the venomous coral snakes (family elapidae) and their mimics (families aniliidae and colubridae). Biological Journal of the Linnean Society, 45(3):235-254.

Schemske, D. W. (1981). Floral convergence and pollinator sharing in two bee-pollinated tropical herbs. Ecology, 62(4):946-954.

Sherratt, T. N. (2006). Spatial mosaic formation through frequency-dependent selection in müllerian mimicry complexes. Journal of Theoretical Biology, 240(2):165-174.

Sherratt, T. N. (2008). The evolution of müllerian mimicry. Die Naturwissenschaften, 95(8):681695.

Springer, V. and Smith-Vaniz, W. (1972). Mimetic relationships involving fishes of the family blenniidae. Smithsonian Contributions Zoology, 112.

Symula, R., Schulte, R., and Summers, K. (2001). Molecular phylogenetic evidence for a mimetic radiation in peruvian poison frogs supports a müllerian mimicry hypothesis. Proceedings of the Royal Society of London. Series B: Biological Sciences, 268(1484):2415-2421.

Willmott, K. R., Robinson Willmott, J. C., Elias, M., and Jiggins, C. D. (2017). Maintaining mimicry diversity: optimal warning colour patterns differ among microhabitats in amazonian clearwing butterflies. Proceedings of the Royal Society B: Biological Sciences, 284(1855):20170744.

Yamaguchi, R. and Iwasa, Y. (2013). Reproductive character displacement by the evolution of female mate choice. Evolutionary Ecology Research, 15(1):25-41. 
Appendix: The limits of evolutionary convergence in

sympatry: reproductive interference and developmental

constraints leading to local diversity in aposematic signals

\section{Model 1: Evolution of mimicry toward a fixed community of defended species}

2

In Section 1.1 we detail how some components of fitness can be approximated using that

4 the genetic covariances of traits and preference are low. In Section 1.2 we detail the computation of the selection vector describing the impact of the selective forces an the

6 evolution of traits and preference. In Section 1.3, in line with the weak selection hypothesis we suppose that the genetic correlation between trait and preference is at equilibrium and 8 estimate this quantity. In Section 1.4 we compute the values of trait and preference that cancel the leading term of the selection vector. In Section 1.5 we study when trait and

10 preference converge toward the point found in the previous section. In Section 1.6 we study the impact of weak or strong female choosiness hypothesis on the phenotypic distance between focal species and the local mimicry ring. In Section 1.7 we detail the impact of each parameter of the phenotypic distance between the focal species and the local mimicry 14 ring. 


\subsection{Low variance approximation}

16

${ }^{24} \quad \int_{\tau} f(\tau) \exp \left[-b(t-\tau)^{2}\right] \mathrm{d} \tau$

26

28

30

32

26

which is equal to

Because we assume that the variance of traits and preference is low we may use approximations in Equations (10) and (13). Here we detail how we obtained these approximations.

The reasoning is similar for each approximation so we only explain how we get an approximation of $\mathcal{D}$ in (10). We recall that $\mathcal{D}$ is defined by

$\mathcal{D}(t)=\lambda N \int_{\tau} f(\tau) \exp \left[-b(t-\tau)^{2}\right] \mathrm{d} \tau+\lambda^{\prime} N^{\prime} \int_{t^{\prime}} g\left(t^{\prime}\right) \exp \left[-b\left(t-t^{\prime}\right)^{2}\right] \mathrm{d} t^{\prime}$

We first approximate the first term of $\mathcal{D}$. We have

$$
=\exp \left[-b(t-\bar{t})^{2}\right] \int_{\tau} f(\tau) \exp [b(2 t-\tau-\bar{t})(\tau-\bar{t})] \mathrm{d} \tau
$$

Using a Taylor expansion of $\exp [b(2 t-\tau-\bar{t})(\tau-\bar{t})]$ we have

$$
\exp \left[-b(t-\bar{t})^{2}\right] \int_{\tau} f(\tau)\left(1+b(2 t-\tau-\bar{t})(\tau-\bar{t})+O\left((\tau-\bar{t})^{2}\right)\right) \mathrm{d} \tau
$$

$$
\exp \left[-b(t-\bar{t})^{2}\right](1-b \operatorname{Var}(t)+O(\operatorname{Var}(t)))
$$

Hence when the variance of $t$ is low the first term of $\mathcal{D}$ can be approximated by

$$
\lambda N \exp \left[-b(t-\bar{t})^{2}\right]
$$

Similar computations for the other term give the approximation in Equation (10).

\subsection{Selection coefficients}

38 In this section we detail how we obtain the expression for the selection vector. 


\subsubsection{Computation of $\beta_{t}$}

42

44

46

48

50

54

\subsubsection{Computation of $\beta_{p}$}

58

For the sake of readability, we first recall the definitions of the quantities under study.

The selective forces acting on the trait value write:

Recall the definition of $W_{\text {pred }}$ in (11). The second term of the right hand side equals

48

$$
\begin{aligned}
2 \frac{\partial}{\partial t} \ln \left(W_{\text {pred }}(t)\right)= & -4 b\left(\left(t-\bar{t}^{\prime}\right) \bar{\lambda}^{\prime} \exp \left[-b\left(t-\bar{t}^{\prime}\right)^{2}\right] N^{\prime}+(t-\bar{t}) \lambda \exp \left[-b(t-\bar{t})^{2}\right] N\right) \\
& \left(\frac{1}{1+\lambda N \exp \left[-b(t-\bar{t})^{2}\right]+\bar{\lambda}^{\prime} \exp \left[-b\left(t-\bar{t}^{\prime}\right)^{2}\right] N^{\prime}-d}\right. \\
& \left.-\frac{1}{1+\lambda N \exp \left[-b(t-\bar{t})^{2}\right]+\bar{\lambda}^{\prime} \exp \left[-b\left(t-\bar{t}^{\prime}\right)^{2}\right] N^{\prime}}\right) .
\end{aligned}
$$

When the parameters $b$ and $d$ are of order $\varepsilon$ (under $\left(H_{w}\right)$ ), we obtain

$$
\beta_{t}=-2 a(\bar{t}-\bar{p})-\frac{4 b d\left(\bar{t}-\bar{t}^{\prime}\right) \bar{\lambda}^{\prime} N^{\prime}}{\left(1+\lambda N+\bar{\lambda}^{\prime} N^{\prime}\right)^{2}}-4 s\left(\bar{t}-t_{a}\right)+O\left(\varepsilon^{3}\right) .
$$

Here again we recall the expression of selective forces acting on the preference value:

$$
\begin{aligned}
\beta_{p} & =\left.\frac{\partial}{\partial p_{f}} \ln W\left(t_{m}, t_{f}, p_{f}\right)\right|_{\left(t_{m}, t_{f}, p_{f}\right)=(\bar{t}, \bar{t}, \bar{p})} \\
& =\left.\frac{\partial}{\partial p_{f}}\left\{-a\left(p_{f}-t_{m}\right)^{2}+\ln W_{R I}\left(p_{f}\right)\right\}\right|_{\left(t_{m}, t_{f}, p_{f}\right)=(\bar{t}, \bar{t}, \bar{p})}
\end{aligned}
$$


.

The last term of the right hand side equals

$$
\begin{aligned}
\frac{\partial}{\partial p_{f}} \ln \left(W_{R I}\left(p_{f}\right)\right) & =-\ln \left(1+c_{R I} \exp \left[a\left(\bar{t}-\bar{t}^{\prime}\right)\left(\bar{t}+\bar{t}^{\prime}-2 p\right)\right] \frac{N^{\prime}}{N}\right) \\
& -\frac{-2 c_{R I} a\left(\bar{t}-\bar{t}^{\prime}\right) \exp \left[a\left(\bar{t}-\bar{t}^{\prime}\right)\left(\bar{t}+\bar{t}^{\prime}-2 p\right)\right] \frac{N^{\prime}}{N}}{1+c_{R I} \exp \left[a\left(\bar{t}-\bar{t}^{\prime}\right)\left(\bar{t}+\bar{t}^{\prime}-2 p\right)\right] \frac{N^{\prime}}{N}} \\
& =\frac{2 c_{R I} a\left(\bar{t}-\bar{t}^{\prime}\right)}{c_{R I}+\exp \left[a\left(\bar{t}-\bar{t}^{\prime}\right)\left(2 p-\bar{t}-\bar{t}^{\prime}\right)\right] \frac{N}{N^{\prime}}} .
\end{aligned}
$$

When the parameters $a$ and $c_{R I}$ are of order $\varepsilon\left(\right.$ under $\left(H_{w}\right)$ ), we finally obtain

$$
\beta_{p}=-2 a(\bar{p}-\bar{t})+2 c_{R I} a\left(\bar{t}-\bar{t}^{\prime}\right) \frac{N^{\prime}}{N}+O\left(\varepsilon^{3}\right)
$$

\subsection{Computation of the matrix of correlation}

In this part we approximate the genetic covariance between trait and preference $C_{t p}$, using the results from Kirkpatrick et al., 2002. Trait and preference are controled by different sets of unlinked loci with additive effects, denoted $T$ and $P$, respectively. For each $i$ in $T$ (resp. $P$ ), we note $\xi_{i}^{t}$ (resp. $\xi_{i}^{p}$ ) the contribution of the locus $i$ on trait (resp. preference) value. The trait and preference values of an individual are then given by

$$
t=\sum_{i \in T} \xi_{i}^{t} \quad \text { and } \quad p=\sum_{i \in P} \xi_{i}^{p} .
$$

As in Lande, 1981 we assume that the distributions of $\xi_{i}^{t}$ and $\xi_{i}^{p}$ are multivariate the matrix of correlation are given by:

$$
G_{t}=\sum_{i, j \in T} C_{i j}, \quad G_{p}=\sum_{i, j \in P} C_{i j} \quad \text { and } \quad C_{t p}=\sum_{i \in T, j \in P} C_{i j}
$$


To compute the change on genetic correlation we need to identify various selection

80

coefficients (see Barton and Turelli, 1991, Kirkpatrick et al., 2002). These coefficients are obtained using the fitness of a mated pair of a male with trait $t_{m}$ and a female with trait $t_{f}$ and preference $p_{f} W\left(t_{m}, t_{f}, p_{f}\right)$ defined in Equation (1).

For simplicity we consider only leading terms in the change in genetic correlation. For $(i, j) \in T \times P$, combining Equations (9), (12), (15) from Kirkpatrick et al. 2002 gives the change in the genetic covariance between loci $i$ and $j$ :

6

$$
\begin{aligned}
\Delta C_{i j}= & -\frac{C_{i j}}{2}+\frac{1}{4} \tilde{a}_{t_{m} t_{m}} \sum_{k, l \in T}\left(C_{i k} C_{j l}+C_{i l} C_{j k}\right)+\frac{1}{4} \tilde{a}_{p_{f} p_{f}} \sum_{k, l \in P}\left(C_{i k} C_{j l}+C_{i l} C_{j k}\right) \\
& +\frac{1}{4} \tilde{a}_{t_{m} p_{f}} \sum_{k \in T, l \in P} C_{i k} C_{j l}+\frac{1}{4} \tilde{a}_{t_{m} p_{f}} \sum_{k \in T, l \in P} C_{i l} C_{j l k}+O\left(\varepsilon^{2}\right)
\end{aligned}
$$

with $\tilde{a}_{\mu \rho}$ for $(\mu, \rho) \in\left\{t_{m}, t_{f}, p_{f}\right\}^{2}$ being the leading term of

$$
a_{\mu \rho}:=\left.\frac{1}{2} \frac{\partial^{2}}{\partial \mu \partial \rho} \ln \left(W\left(t_{m}, t_{f}, p_{f}\right)\right)\right|_{\left(t_{m}, t_{f}, p_{f}\right)=(\bar{t}, \bar{t}, \bar{p})}
$$

the selection coefficient calculated from the fitness of a mated pair of a male with trait $t_{m}$ and a female with trait $t_{f}$ and preference $p_{f}$. The expressions of these coefficients are:

$$
a_{p_{f} p_{f}}=-a-\frac{2 c_{R I} a^{2}\left(\bar{t}-\bar{t}^{\prime}\right)^{2} \frac{N}{N^{\prime}} \exp \left[a\left(\bar{t}-\bar{t}^{\prime}\right)\left(2 \bar{p}-\bar{t}-\bar{t}^{\prime}\right)\right]}{\left(c_{R I}+\frac{N}{N^{\prime}} \exp \left[a\left(\bar{t}-\bar{t}^{\prime}\right)\left(2 \bar{p}-\bar{t}-\bar{t}^{\prime}\right)\right]\right)^{2}}
$$


94

96

$$
\begin{aligned}
a_{t_{m} t_{m}}= & -a-s+2 b^{2}\left(\bar{\lambda}^{\prime} N^{\prime}\left(1+\left(\bar{t}-\bar{t}^{\prime}\right)^{2}\right) \exp \left[b\left(\bar{t}-\bar{t}^{\prime}\right)^{2}\right]+\lambda N\right) \\
& \times\left(\frac{1}{1+\lambda N+\bar{\lambda}^{\prime} N^{\prime} \exp \left[b\left(\bar{t}-\bar{t}^{\prime}\right)^{2}\right]-d}-\frac{1}{1+\lambda N+\bar{\lambda}^{\prime} N^{\prime} \exp \left[b\left(\bar{t}-\bar{t}^{\prime}\right)^{2}\right]}\right) \\
& +\left(2 b \bar{\lambda}^{\prime} N^{\prime}\left(\bar{t}-\bar{t}^{\prime}\right) \exp \left[b\left(\bar{t}-\bar{t}^{\prime}\right)^{2}\right]\right)^{2} \\
& \times\left(\frac{1}{\left(1+\lambda N+\bar{\lambda}^{\prime} N^{\prime} \exp \left[b\left(\bar{t}-\bar{t}^{\prime}\right)^{2}\right]-d\right)^{2}}-\frac{1}{\left(1+\lambda N+\bar{\lambda}^{\prime} N^{\prime} \exp \left[b\left(\bar{t}-\bar{t}^{\prime}\right)^{2}\right]\right)^{2}}\right),
\end{aligned}
$$

and

$$
a_{t_{m} p_{f}}=2 a .
$$

A Taylor expansion gives $\tilde{a}_{p_{f} p_{f}}=-a, \tilde{a}_{t_{m} t_{m}}=-a$ and $\tilde{a}_{t_{m} p_{f}}=2 a$.

By summing Equations (A5) over each $i, j$ in $T$ and $P$ we obtain:

$$
\Delta C_{t p}=-\frac{C_{t p}}{2}-\frac{1}{2} a G_{t} C_{t p}-\frac{1}{2} a G_{p} C_{t p}+\frac{1}{2} a G_{t} G_{p}+\frac{1}{2} a C_{t p}^{2}+O\left(\varepsilon^{2}\right) .
$$

For the sake of simplicity we assumed that the genetic correlations between traits and preferences are at equilibrium (as in Barton and Turelli, 1991, Pomiankowski and Iwasa, 1993 ). We obtain from (A6 that the value at equilibrium is given by

$$
C_{t p}^{*}=\frac{1+a\left(G_{p}+G_{t}\right)-\sqrt{-4 a^{2} G_{p} G_{t}+\left(1+a\left(G_{p}+G_{t}\right)\right)^{2}}}{2 a} .
$$

Because the genetic variances of trait and preference are low we have

$$
C_{t p}^{*} \approx a G_{t} G_{p} .
$$


When we relax the hypothesis $a=O(\varepsilon)$ and $b=O(\varepsilon)$ the previous calculation still

114 stands, except that $\tilde{a}_{p_{f} p_{f}}, \tilde{a}_{t_{m} t_{m}}$ and $\tilde{a}_{t_{m} p_{f}}$ are of order 1 instead of being of order $\varepsilon$.

\subsection{Quasi equilibria}

We terms of $\Delta \bar{t}$ and $\Delta \bar{p}$ are null. We remind that we assume weak female choosiness and predator discrimination (default hypothesis $\left(H_{w}\right)$ ). The leading terms of $\Delta \bar{t}^{*}$ and $\Delta \bar{p}^{*}$ are thus $\tilde{\beta}_{t}=\tilde{\beta}_{p}=0$ with

$$
\begin{aligned}
& \tilde{\beta}_{t}=-2 a\left(\bar{t}^{*}-\bar{p}^{*}\right)-\frac{4 b d\left(\bar{t}^{*}-\bar{t}^{\prime}\right) \bar{\lambda}^{\prime} N^{\prime}}{\left(1+\lambda N+\bar{\lambda}^{\prime} N^{\prime}\right)^{2}}-4 s\left(\bar{t}^{*}-t_{a}\right), \\
& \tilde{\beta}_{p}=-2 a\left(\bar{p}^{*}-\bar{t}^{*}\right)+2 c_{R I} a\left(\bar{t}^{*}-\bar{t}^{\prime}\right) \frac{N^{\prime}}{N} .
\end{aligned}
$$

We call such points quasi equilibria because they are not equilibrium point of Equation (6) but only cancel the term of leading order.

Lemma 1. If

$$
\frac{2 b d \bar{\lambda}^{\prime} N^{\prime}}{\left(\lambda N+\bar{\lambda}^{\prime} N^{\prime}+1\right)^{2}}+2 s-\frac{a c_{R I} N^{\prime}}{N} \neq 0
$$

there is one quasi equilibrium point $\left(\bar{t}^{*}, \bar{p}^{*}\right)$, where

$$
\bar{t}^{*}=\frac{\frac{2 b d \bar{\lambda}^{\prime} N^{\prime}}{\left(1+\lambda N+\bar{\lambda}^{\prime} N^{\prime}\right)^{2}} \bar{t}^{\prime}+2 s t_{a}-\frac{a c_{R I} N^{\prime}}{N} \bar{t}^{\prime}}{\frac{2 b d \bar{\lambda}^{\prime} N^{\prime}}{\left(1+\lambda N+\bar{\lambda}^{\prime} N^{\prime}\right)^{2}}+2 s-\frac{a c_{R I} N^{\prime}}{N}} \text { and } \bar{p}^{*}=\bar{t}^{*}+c_{R I}\left(\bar{t}^{*}-\bar{t}^{\prime}\right) \frac{N^{\prime}}{N} .
$$

Proof. Assume that $a \neq 0$. From the definitions of $\tilde{\beta}_{t}$ and $\tilde{\beta}_{p}$ in A7 and (A8), we deduce that the values of trait and preference $\left(\bar{t}^{*}, \bar{p}^{*}\right)$ for which the selections on trait and preference are null satisfy:

$$
\tilde{\beta}_{t}=0 \Longleftrightarrow a\left(\bar{p}^{*}-\bar{t}^{*}\right)=\frac{2 b d\left(\bar{t}^{*}-\bar{t}^{\prime}\right) \bar{\lambda}^{\prime} N^{\prime}}{\left(1+\lambda N+\bar{\lambda}^{\prime} N^{\prime}\right)^{2}}+2 s\left(\bar{t}^{*}-t_{a}\right)
$$


and

$$
\tilde{\beta}_{p}=0 \Longleftrightarrow a\left(\bar{p}^{*}-\bar{t}^{*}\right)=a c_{R I}\left(\bar{t}^{*}-\bar{t}^{\prime}\right) \frac{N^{\prime}}{N}
$$

From A11 and $\mathrm{A} 12$ we derivate:

$$
\frac{2 b d\left(\bar{t}^{*}-\bar{t}^{\prime}\right) \bar{\lambda}^{\prime} N^{\prime}}{\left(1+\lambda N+\bar{\lambda}^{\prime} N^{\prime}\right)^{2}}+2 s\left(\bar{t}^{*}-t_{a}\right)=a c_{R I}\left(\bar{t}^{*}-\bar{t}^{\prime}\right) \frac{N^{\prime}}{N} .
$$

138

By factorizing by $\bar{t}$ we get:

$$
\bar{t}^{*}\left(\frac{2 b d \bar{\lambda}^{\prime} N^{\prime}}{\left(1+\lambda N+\bar{\lambda}^{\prime} N^{\prime}\right)^{2}}+2 s-a c_{R I} \frac{N^{\prime}}{N}\right)=\bar{t}^{\prime}\left(\frac{2 b d \bar{\lambda}^{\prime} N^{\prime}}{\left(1+\lambda N+\bar{\lambda}^{\prime} N^{\prime}\right)^{2}}-a c_{R I} \frac{N^{\prime}}{N}\right)+2 s t_{a} .
$$

Using A9, we obtain the value of $\bar{t}^{*}$ given in A10. From A12 we deduce the associated mean preference and conclude the proof.

\subsection{Fast and slow dynamics.}

In this section we study when trait and preference in the focal species converge towards

144

146

Lemma 2. 1. If

We suppose that the parameters are such as $\frac{2 b d \bar{\lambda}^{\prime} N^{\prime}}{\left(\lambda N+\bar{\lambda}^{\prime} N^{\prime}+1\right)^{2}}+2 s-\frac{a c_{R I} N^{\prime}}{N}$ is not of an order inferior to $\varepsilon^{2}$. We may have two long term behaviours. In case of convergence (case 1. of Lemma 22, the trajectories quickly approach the line $\bar{t}=\bar{p}$, before evolving according to a slower dynamic along this line (see the proof of Lemma 2 and Figures 1 and 2).

$$
\frac{2 b d \bar{\lambda}^{\prime} N^{\prime}}{\left(\lambda N+\bar{\lambda}^{\prime} N^{\prime}+1\right)^{2}}+2 s-\frac{a c_{R I} N^{\prime}}{N}>0
$$


the quantities $\left(\bar{t}-\bar{t}^{*}\right)$ and $\left(\bar{p}-\bar{p}^{*}\right)$ become of order $\sqrt{\varepsilon}$ when the number of generations goes to infinity, where the values of $\bar{t}^{*}$ and $\bar{p}^{*}$ have been given in (A10).

2. If

$$
\frac{2 b d \bar{\lambda}^{\prime} N^{\prime}}{\left(\lambda N+\bar{\lambda}^{\prime} N^{\prime}+1\right)^{2}}+2 s-\frac{a c_{R I} N^{\prime}}{N}<0,
$$

trait and preference become very large.

Proof. As we have said, we can then decompose the dynamics into two steps. In the first one the leading order terms of the selection coefficient are the terms describing sexual selection and cost of choosiness. In this case we can approximate $\Delta(\bar{t}-\bar{p})^{2}$.

$$
\begin{aligned}
\Delta(\bar{t}-\bar{p})^{2} & =(\bar{t}+\Delta \bar{t}-\bar{p}-\Delta \bar{p})^{2}-(\bar{t}-\bar{p})^{2} \\
& =2(\bar{t}-\bar{p})(\Delta \bar{t}-\Delta \bar{p})+(\Delta \bar{t}-\Delta \bar{p})^{2} \\
& =2(\bar{t}-\bar{p})\left(\frac{1}{2} G_{t} \beta_{t}-\frac{1}{2} G_{p} \beta_{p}\right)+\left(\frac{1}{2} G_{t} \beta_{t}-\frac{1}{2} G_{p} \beta_{p}\right)^{2} \\
& =-(\bar{t}-\bar{p})\left(2 a\left(G_{t}+G_{p}\right)(\bar{t}-\bar{p})-a^{2}\left(G_{p}+G_{t}\right)^{2}(\bar{t}-\bar{p})\right. \\
& \left.-2\left(\frac{a c_{R I} G_{p} N^{\prime}\left(\bar{t}^{\prime}-\bar{t}\right)}{N}+\frac{2 b d G_{t} \bar{\lambda}^{\prime} N^{\prime}\left(\bar{t}^{\prime}-\bar{t}\right)}{\left(l N+\bar{\lambda}^{\prime} N^{\prime}+1\right)^{2}}+G_{t} s\left(t_{a}-\bar{t}\right)\right)\right)+O\left(\varepsilon^{3}\right),
\end{aligned}
$$

where we used (6) for the third equality and (A1) and (A2) for the last one. As by assumption, $a, c_{R I}, b$ and $d$ are of order $\varepsilon$, and $s$ of order $\varepsilon^{2}$, we obtain

$$
\Delta(\bar{t}-\bar{p})^{2}=-(\bar{t}-\bar{p})\left(2 a\left(G_{t}+G_{p}\right)(\bar{t}-\bar{p})+O\left(\varepsilon^{2}\right)\right)+O\left(\varepsilon^{3}\right) .
$$

Denote by $r_{n}$ the value of $(\bar{t}-\bar{p})^{2}$ at generation $n$. We will prove the following statement:

$$
\text { There exists } \alpha>0 \text { such that for } n \text { large enough, } r_{n} \leq 2 \alpha \varepsilon^{2} \text {. }
$$

If we introduce the parameter$$
\text { (1) }
$$

$$
\mathcal{A}:=1-2 a\left(G_{t}+G_{p}\right)
$$


Equation A14 may be rewritten:

$$
r_{n+1}=\mathcal{A} r_{n}+\sqrt{r_{n}} f_{n}(1, \varepsilon)+f_{n}(2, \varepsilon)
$$

for every $n \in \mathbb{N}\left|f_{n}(1, \varepsilon)\right| \leq c_{1} \varepsilon^{2},\left|f_{n}(2, \varepsilon)\right| \leq c_{2} \varepsilon^{3}$ where $c_{1}$ and $c_{2}$ are finite constants.

$$
\rho_{n}:=r_{n}-r_{0} \mathcal{A}^{n}
$$

As $a$ is of order $\varepsilon$, and $G_{t}$ and $G_{p}$ of order one, there exists a positive and finite constant $C_{\mathcal{A}}$ such that for $\varepsilon$ small enough,

$$
0<\mathcal{A}<1-C_{\mathcal{A}} \varepsilon
$$

We will choose in the sequel a positive real number $\alpha$ satisfying

$$
\sqrt{\frac{2}{\alpha}} c_{1}+\frac{c_{2}}{\alpha}<C_{\mathcal{A}}
$$

and

$$
\frac{c_{1}}{\sqrt{\alpha}}+\frac{\sqrt{\alpha} c_{1}+c_{2}}{\alpha}<C_{\mathcal{A}}
$$

As $0<\mathcal{A}<1$, we see that for $n$ large enough, $r_{0} \mathcal{A}^{n} \leq \alpha \varepsilon^{2}$. Thus to prove (A15), it 172 is enough to prove that for $n$ large enough, $\left|\rho_{n}\right| \leq \alpha \varepsilon^{2}$. We obtain for $\rho$ the recurrence equation

$$
\rho_{n+1}=\mathcal{A} \rho_{n}+\sqrt{\rho_{n}+r_{0} \mathcal{A}^{n}} f_{n}(1, \varepsilon)+f_{n}(2, \varepsilon) .
$$

Assume first that $\left|\rho_{n}\right| \leq \alpha \varepsilon^{2}$. In this case,

176

78

$$
\begin{aligned}
\left|\rho_{n+1}\right| & \leq \mathcal{A} \alpha \varepsilon^{2}+\sqrt{2 \alpha \varepsilon^{2}} c_{1} \varepsilon^{2}+c_{2} \varepsilon^{3} \\
& =\alpha \varepsilon^{2}\left(\mathcal{A}+\sqrt{\frac{2}{\alpha}} c_{1} \varepsilon+\frac{c_{2}}{\alpha} \varepsilon\right) \leq \alpha \varepsilon^{2}
\end{aligned}
$$


thanks to A16 provided that $\varepsilon$ is small enough. Hence it is enough to prove that there

exists one $n$ such that $\rho_{n} \leq \alpha \varepsilon^{2}$, and the inequality will hold for later generations.

Now assume that $\rho_{n}>\alpha \varepsilon^{2}$. Using that

82

$$
\sqrt{\rho_{n}+r_{0} \mathcal{A}^{n}} \leq \sqrt{\left|\rho_{n}\right|}+\sqrt{r_{0} \mathcal{A}^{n}} \leq \sqrt{\left|\rho_{n}\right|}+\sqrt{\alpha} \varepsilon
$$

for $n$ large enough, we obtain from A18

8

$$
\begin{aligned}
\left|\rho_{n+1}\right| & \leq \mathcal{A}\left|\rho_{n}\right|+\sqrt{\left|\rho_{n}\right|} c_{1} \varepsilon^{2}+\left(\sqrt{\alpha} c_{1}+c_{2}\right) \varepsilon^{3} \\
& \leq \mathcal{A}\left|\rho_{n}\right|+\sqrt{\left|\rho_{n}\right|} c_{1} \sqrt{\frac{\left|\rho_{n}\right|}{\alpha}} \varepsilon+\left(\sqrt{\alpha} c_{1}+c_{2}\right) \frac{\left|\rho_{n}\right|}{\alpha} \varepsilon \\
& =\left|\rho_{n}\right|\left(\mathcal{A}+\frac{c_{1}}{\sqrt{\alpha}} \varepsilon+\frac{\sqrt{\alpha} c_{1}+c_{2}}{\alpha} \varepsilon\right) .
\end{aligned}
$$

Due to A17, the term in bracket belongs to $(0,1)$ if $\varepsilon$ is small enough. We conclude that there exists $n_{0}$ such that $\left|\rho_{n}\right| \leq \alpha \varepsilon^{2}$ for any $n \geq n_{0}$. This concludes the proof of (A15) and of the fast convergent phase.

We now want to prove that $\bar{t}$ becomes close to $\bar{t}^{*}$, and $\bar{p}$ to $\bar{p}^{*}$. To this aim, we look at the variation of

$$
\frac{\left(\bar{t}-\bar{t}^{*}\right)^{2}}{G_{t}}+\frac{\left(\bar{p}-\bar{p}^{*}\right)^{2}}{G_{p}}
$$

where the values of $\bar{t}^{*}$ and $\bar{p}^{*}$ are the ones defined in A10. By definition,

$$
\Delta\left(\frac{\left(\bar{t}-\bar{t}^{*}\right)^{2}}{G_{t}}+\frac{\left(\bar{p}-\bar{p}^{*}\right)^{2}}{G_{p}}\right)=\frac{2 \Delta \bar{t}\left(\bar{t}-\bar{t}^{*}\right)+(\Delta \bar{t})^{2}}{G_{t}}+\frac{2 \Delta \bar{p}\left(\bar{p}-\bar{p}^{*}\right)+(\Delta \bar{p})^{2}}{G_{p}}
$$

When $\bar{t}-\bar{p}$ is of order $\varepsilon, \Delta \bar{t}$ and $\Delta \bar{p}$ are of order $\varepsilon^{2}$. We then neglect the terms proportional to $(\Delta \bar{t})^{2}$ and $(\Delta \bar{p})^{2}$. This yields

$$
\begin{aligned}
\Delta\left(\frac{\left(\bar{t}-\bar{t}^{*}\right)^{2}}{G_{t}}+\frac{\left(\bar{p}-\bar{p}^{*}\right)^{2}}{G_{p}}\right) & =\frac{2 \Delta \bar{t}\left(\bar{t}-\bar{t}^{*}\right)}{G_{t}}+\frac{2 \Delta \bar{p}\left(\bar{p}-\bar{p}^{*}\right)}{G_{p}}+O\left(\varepsilon^{4}\right) \\
& =\beta_{t}\left(\bar{t}-\bar{t}^{*}\right)+\beta_{p}\left(\bar{p}-\bar{p}^{*}\right)+O\left(\varepsilon^{4}\right)
\end{aligned}
$$


where we used (6). Now notice that

208

$$
\bar{t}-\bar{t}^{*}=\bar{t}-\bar{p}+\bar{p}-\bar{p}^{*}+\bar{p}^{*}-\bar{t}^{*}=\bar{p}-\bar{p}^{*}+O(\varepsilon)=\frac{\bar{t}-\bar{t}^{*}}{2}+\frac{\bar{p}-\bar{p}^{*}}{2}+O(\varepsilon),
$$

as $\bar{p}-\bar{t}$ is of order $\varepsilon$ and $\bar{p}^{*}-\bar{t}^{*}$ of order $\varepsilon$. We can make the same computation for $\bar{p}-\bar{p}^{*}$. Adding that $\beta_{t}$ and $\beta_{p}$ are of order $\varepsilon^{2}$, we obtain:

$$
\Delta\left(\frac{\left(\bar{t}-\bar{t}^{*}\right)^{2}}{G_{t}}+\frac{\left(\bar{p}-\bar{p}^{*}\right)^{2}}{G_{p}}\right)=\left(\beta_{t}+\beta_{p}\right) \frac{\bar{t}-\bar{t}^{*}}{2}+\left(\beta_{t}+\beta_{p}\right) \frac{\bar{p}-\bar{p}^{*}}{2}+O\left(\varepsilon^{3}\right) .
$$

Using (A1) and A2 and simplifying, we get:

$$
\beta_{t}+\beta_{p}=-\frac{4 b d\left(\bar{t}-\bar{t}^{\prime}\right) \bar{\lambda}^{\prime} N^{\prime}}{\left(1+\lambda N+\bar{\lambda}^{\prime} N^{\prime}\right)^{2}}-4 s\left(\bar{t}-t_{a}\right)+2 c_{R I} a\left(\bar{t}-\bar{t}^{\prime}\right) \frac{N^{\prime}}{N}+O\left(\varepsilon^{3}\right) .
$$

By substracting $-\frac{4 b d\left(\bar{t}^{*}-\bar{t}^{\prime}\right) \bar{\lambda}^{\prime} N^{\prime}}{\left(1+\lambda N+\bar{\lambda}^{\prime} N^{\prime}\right)^{2}}-2 s\left(\bar{t}^{*}-t_{a}\right)+2 c_{R I} a\left(\bar{t}^{*}-\bar{t}^{\prime}\right) \frac{N^{\prime}}{N}$ which is equal to zero (because $\left.\beta_{t}\right|_{(t, p)=\left(\bar{t}^{*}, \bar{p}^{*}\right)}=0$ and $\left.\beta_{p}\right|_{(t, p)=\left(\bar{t}^{*}, \bar{p}^{*}\right)}=0$, we get:

$$
\begin{aligned}
\beta_{t}+\beta_{p} & =-\left(\frac{2 b d \bar{\lambda}^{\prime} N^{\prime}}{\left(1+\lambda N+\bar{\lambda}^{\prime} N^{\prime}\right)^{2}}+2 s-a c_{R I} \frac{N^{\prime}}{N}\right) 2\left(\bar{t}-\bar{t}^{*}\right)+O\left(\varepsilon^{3}\right) \\
& =-\left(\frac{2 b d \bar{\lambda}^{\prime} N^{\prime}}{\left(1+\lambda N+\bar{\lambda}^{\prime} N^{\prime}\right)^{2}}+2 s-a c_{R I} \frac{N^{\prime}}{N}\right) 2\left(\bar{p}-\bar{p}^{*}\right)+O\left(\varepsilon^{3}\right),
\end{aligned}
$$

where we used A19. Replacing $\beta_{t}+\beta_{p}$ using A21 and A22 into A20 yield:

$$
\Delta\left(\frac{\left(\bar{t}-\bar{t}^{*}\right)^{2}}{G_{t}}+\frac{\left(\bar{p}-\bar{p}^{*}\right)^{2}}{G_{p}}\right)=-A\left(\left(\bar{t}-\bar{t}^{*}\right)^{2}+\left(\bar{p}-\bar{p}^{*}\right)^{2}\right)+O\left(\varepsilon^{3}\right),
$$

with

$$
A:=\left(\frac{2 b d \bar{\lambda}^{\prime} N^{\prime}}{\left(1+\lambda N+\bar{\lambda}^{\prime} N^{\prime}\right)^{2}}+2 s-a c_{R I} \frac{N^{\prime}}{N}\right) .
$$


First case: $A>0$

We suppose that the parameters are such that $A$ is not of an order inferior to $\varepsilon^{2}$. Using computations similar to the ones derived to prove (A15), we may prove that after a number of generations large enough, $\left(\bar{t}-\bar{t}^{*}\right)^{2}$ and $\left(\bar{p}-\bar{p}^{*}\right)^{2}$ become of order $\varepsilon$, and thus $\bar{t}-\bar{t}^{*}$ and $\bar{p}-\bar{p}^{*}$ become of order $\varepsilon^{1 / 2}$.

To illustrate this result we simulated two trajectories of the system $(6)\left(\left(\bar{t}_{n}^{1}, \bar{p}_{n}^{1}\right)\right)_{n \in \mathbb{N}}$ and $\left(\left(\bar{t}_{n}^{2}, \bar{p}_{n}^{2}\right)\right)_{n \in \mathbb{N}}$ for two different initial conditions: $\left(t^{i 1}, p^{i 1}\right)$ and $\left(t^{i 2}, p^{i 2}\right)$. The trajectories are shown in Figure 1 and the parameter values are given in the caption. The parameter values correspond to the weak selection hypothesis and are such that $A>0$.

The fast and slow dynamics are visible in Figure 1(a) where the trajectories quickly become close to the line defined by $\bar{t}=\bar{p}$ and then slowly converge toward the quasi equilibrium points. We noted $\left(\bar{t}^{e q}, \bar{p}^{e q}\right)$ the equilibrium point reached by the two simulated trajectories.

\section{Second case: $A<0$.}

Recall (A23) and that we assumed that $A$ is not of order inferior to $\varepsilon^{2}$. This entails that $\left(\bar{t}-\bar{t}^{*}\right)^{2} / G_{t}+\left(\bar{p}-\bar{p}^{*}\right)^{2} / G_{p}$ goes to infinity. However when $t-\bar{t}^{\prime}$ or $p-\bar{p}^{\prime}$ becomes high (for example of order $1 / \varepsilon$ ) the approximation

$$
\exp \left[a\left(t-\bar{t}^{\prime}\right)\left(2 p-t-\bar{t}^{\prime}\right)\right]=a\left(t-\bar{t}^{\prime}\right)\left(2 p-t-\bar{t}^{\prime}\right)+O\left(\varepsilon^{2}\right)
$$

does not stand. Therefore from a certain number of generations the approximation does not correctly describe the evolution of trait and preference. We can only conclude that trait and preference become very large. This is illustrated by Figure 2 which shows a simulation of two trajectories when $A<0$. 


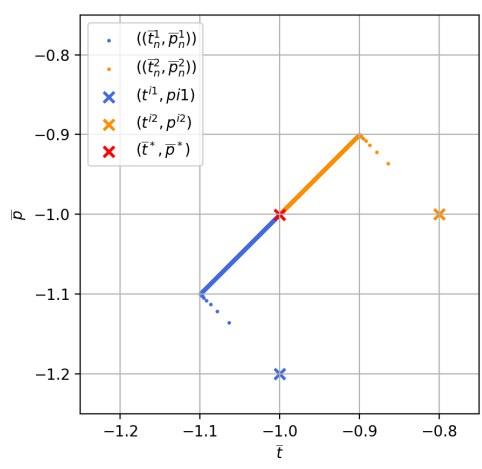

(a)

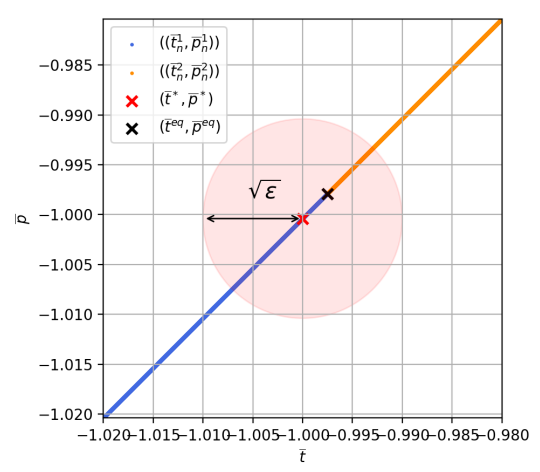

(b)

Figure 1: (a) Evolution of mean trait value $\bar{t}$ and mean female preference value $\bar{p}$ for two different initial conditions. The blue (resp. orange) points represent the numerical trajectories for the initial condition $\left(t^{i 1}, p^{i 1}\right)$ (resp. $\left.\left(t^{i 2}, p^{i 2}\right)\right)(\mathrm{b})$ Zoom on the previous figure. The red circle has for center the quasi equilibrium point $\left(\bar{t}^{*}, \bar{p}^{*}\right)$ and for radius $\sqrt{\varepsilon}$. This illustrates that the numerical steady point $\left(\bar{t}^{e q}, \bar{p}^{e q}\right)$ is at a distance of order $\sqrt{\varepsilon}$. The trajectories were obtained with the parameter values: $\left(t^{i 1}, p^{i 1}\right)=(-1,-1.2)$, $\left(t^{i 2}, p^{i 2}\right)=(-0.8,-1), \mathrm{a}=10^{-4}, \mathrm{~b}=2 \times 10^{-4}, d=2 \times 10^{-4}, \lambda=0.1, \bar{\lambda}^{\prime}=0.1, N=10$, $N_{p}=20, c_{R I}=10^{-4}, s=10^{-8}, \bar{t}^{\prime}=1$ and $t_{a}=0$. The trajectories are computed over $1,000,000$ generations.

\subsection{Effect of weak or strong female choosiness on the phenotypic dis- tances between the focal species and the local mimicry ring}

Previously we obtained an analytic approximation of the phenotypic distance between the

focal species and the local mimicry ring when the strengths of female choosiness $(a)$ and predator discrimination $(b)$ were weak (i.e. $a=O(\varepsilon)$ and $\left.b=O(\varepsilon)\left(H_{w}\right)\right)$. To obtain this approximation we also assumed that the strength of developmental and selective constraints 


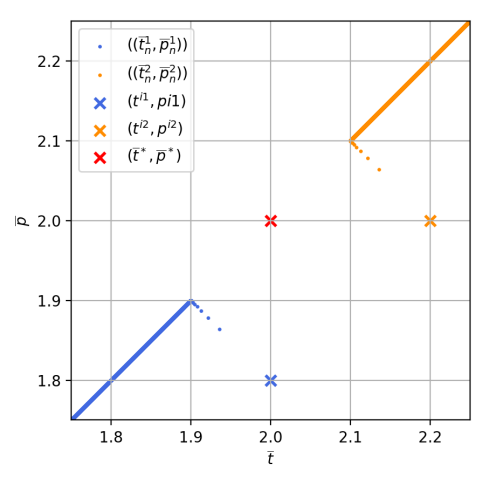

Figure 2: Evolution of mean trait value $\bar{t}$ and mean female preference value $\bar{p}$ for two different initial conditions. The blue (resp. orange) points represent the numerical trajectories for the initial condition $\left(t^{i 1}, p^{i 1}\right)$ (resp. $\left.\left(t^{i 2}, p^{i 2}\right)\right)$. The trajectories were obtained with the parameter values: $\left(t^{i 1}, p^{i 1}\right)=(-1,-1.2),\left(t^{i 2}, p^{i 2}\right)=(-0.8,-1), \mathrm{a}=10^{-4}, \mathrm{~b}=2 \times 10^{-4}$, $d=2 \times 10^{-4}, \lambda=0.1, \bar{\lambda}^{\prime}=0.1, N=10, N_{p}=20, c_{R I}=10^{-4}, s=0.25 \times 10^{-8}, \bar{t}^{\prime}=1$ and $t_{a}=0$. The trajectories are computed over 1,000,000 generations.

has the same order of magnitude as selection coefficients due to predation and reproductive

interference (i.e. $s=O\left(\varepsilon^{2}\right)$ ). Under this assumption, the phenotypic distance between the focal species and the local mimicry ring becomes very large (see Figure 3) when

$$
c_{R I} \geq \frac{2 N}{a N^{\prime}}\left(\frac{b d \bar{\lambda}^{\prime} N^{\prime}}{\left(1+\lambda N+\bar{\lambda}^{\prime} N^{\prime}\right)^{2}}+s\right) .
$$

As explained in the main manuscript, this unrealistic divergence (under the weak choosiness hypothesis $(a=O(\varepsilon)))$ suggests that reproductive interference promotes strong phenotype divergence between the focal species and the local mimicry ring. Under weak choosiness, only a large distance between females preference and the trait displayed by the 
local mimicry ring leads to a reduction of fitness cost generated by RI. Because females preference generates sexual selection on males trait, the phenotype divergence between the focal species and the local mimicry ring becomes high. When we relax the weak choosiness hypothesis $\left(H_{s}\right)$ and use numerical simulations, then a great cost of reproductive interference provokes large but still of order 1 phenotypic distance between the focal species and the local mimicry ring (see Figure 3). However, we observe that the lower female choosiness, the higher is phenotypic distance when the cost of reproductive interference increases. As explained above, when females are not very choosy, reproductive interference favors the evolution of larger phenotypic distances limiting heterospecific mating. 


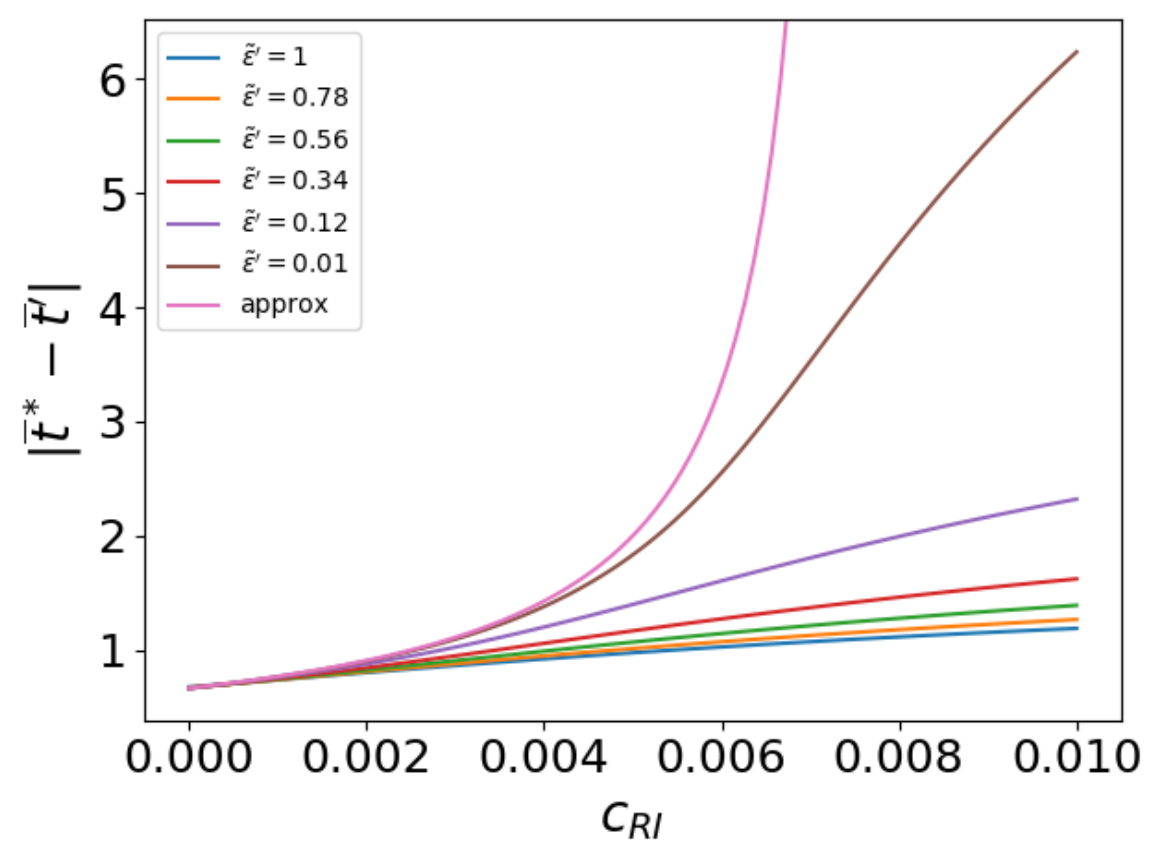

Figure 3: Influence of the cost generated by reproductive interference $c_{R I}$ on the phenotypic distances between the focal species and the local mimicry rings, using the analytical approximation ('approx' curve) or numerical simulations. Different values of female choosiness $(a)$, predator discrimination coefficient $(b)$ and strength of selective and developmental constraints $(s)$ are assumed for numerical simulations. This illustrate that curves obtained by numerical simulations tend to look similar to those using the analytical approximation where parameters values tends to satisfies $\left(H_{w}\right)$. We assume: $a=\tilde{\varepsilon}^{\prime}, b=\tilde{\varepsilon}^{\prime}, s=0.5 \tilde{\varepsilon} \tilde{\varepsilon}^{\prime}, d=2 \tilde{\varepsilon}, \lambda=0.1, \bar{\lambda}^{\prime}=0.1, N=10, N^{\prime}=20, t_{a}=0, \bar{t}^{\prime}=1$.

\subsection{Effect of different parameters on the level of trait divergence $\left|\bar{t}^{*}-\bar{t}^{\prime}\right|$}

We then aim at disentangling the effects of the different parameters of the model on trait divergence. We thus focus on the case where $A>0$ (recall definition (A24)). In this case, 
assuming weak female choosiness and predator discrimination $\left(H_{w}\right)$, we know that the phenotypic distance between the focal species and the local mimicry ring, $\left|\bar{t}^{*}-\bar{t}^{\prime}\right|$, equals

$$
\left|\bar{t}^{*}-\bar{t}^{\prime}\right|=\frac{2 s\left|t_{a}-\bar{t}^{\prime}\right|}{\frac{2 b d \bar{\lambda}^{\prime} N^{\prime}}{\left(1+\lambda N+\bar{\lambda}^{\prime} N^{\prime}\right)^{2}}+2 s-a c_{R I} \frac{N^{\prime}}{N}},
$$

allowing to investigate the relative impact of the different parameters on trait divergence. We also run numerical simulations to investigate the effect of the different parameters assuming strong females preference and predator discrimination (i.e. $a=O(1)$ and $b=$ $\left.O(1)\left(H_{s}\right)\right)$.

\subsubsection{Effect of reproductive interference}

Assuming weak female choosiness and predator discrimination $\left(H_{w}\right)$, the impact of the strength of reproductive interference on the phenotypic distance between the focal species

Hence the level of divergence increases with the strength of RI. A similar effect is observed assuming strong female choosiness and predator discrimination $\left(H_{s}\right)$ (see Figure 4(a)). Similarly, assuming weak female choosiness and predator discrimination $\left(H_{w}\right)$

$$
\frac{\partial\left|\bar{t}^{*}-\bar{t}^{\prime}\right|}{\partial c_{R I}}=\frac{2 s a \frac{N^{\prime}}{N}\left|t_{a}-\bar{t}^{\prime}\right|}{\left(\frac{2 b d \bar{\lambda}^{\prime} N^{\prime}}{\left(1+\lambda N+\bar{\lambda}^{\prime} N^{\prime}\right)^{2}}+2 s-a c_{R I} \frac{N^{\prime}}{N}\right)^{2}}>0 .
$$
and the local mimicry ring is given by the sign of $\partial\left|\bar{t}^{*}-\bar{t}^{\prime}\right| / \partial c_{R I}$. In our case,

$$
\frac{\partial\left|\bar{t}^{*}-\bar{t}^{\prime}\right|}{\partial a}=\frac{2 s c_{R I} \frac{N^{\prime}}{N}\left|t_{a}-\bar{t}^{\prime}\right|}{\left(\frac{2 b d \bar{\lambda}^{\prime} N^{\prime}}{\left(1+\lambda N+\bar{\lambda}^{\prime} N^{\prime}\right)^{2}}+2 s-a c_{R I} \frac{N^{\prime}}{N}\right)^{2}}>0
$$

thus the phenotypic distance (along with the strength of selection due to RI) increases with the strength of female choosiness. However, when female choosiness is strong and exceeds a threshold, females are able to distinguish males from their own species from individuals from the local mimicry rings, which limits divergence of the trait in the focal species (see 
288 divergent selection exerted by RI.

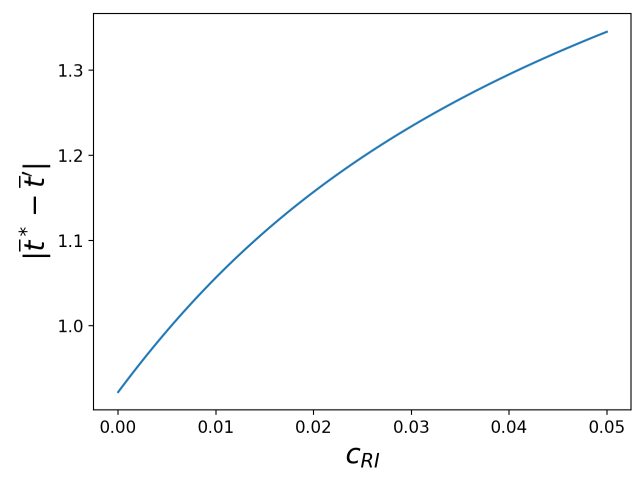

(a)

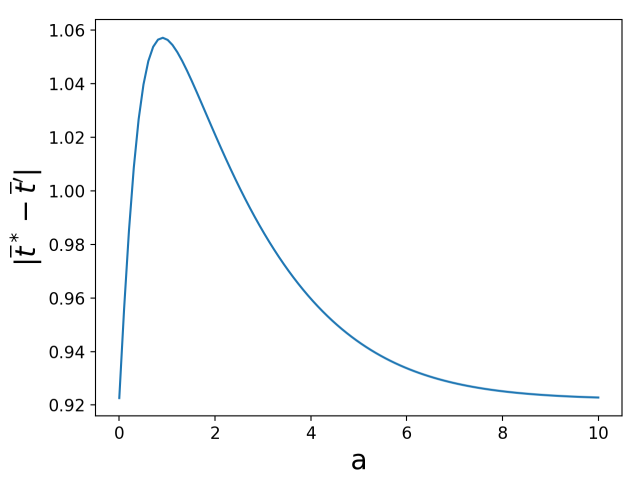

(b)

Figure 4: Influence of the strength of (a) reproductive interference $c_{R I}$ and of (b) female choosiness $a$ on the phenotypic distances between the focal species and the local mimicry rings, assuming strong female choosiness and predator discrimination. The default parameters values are as follows: $a=1, c_{R I}=0.01, b=1$, $d=0.02, \lambda=0.1, \bar{\lambda}^{\prime}=0.1, N=10, N^{\prime}=20, s=0.0025, t_{a}=0, \bar{t}^{\prime}=1$.

\subsubsection{Effect of predation}

When $a$ and $b$ are of order $\varepsilon\left(H_{w}\right)$,

$$
\frac{\partial\left|\bar{t}^{*}-\bar{t}^{\prime}\right|}{\partial d}=\frac{-2 b \bar{\lambda}^{\prime} N^{\prime}}{\left(1+\lambda N+\bar{\lambda}^{\prime} N^{\prime}\right)^{2}} \frac{2 s\left|t_{a}-\bar{t}^{\prime}\right|}{\left(\frac{2 b d \bar{\lambda}^{\prime} N^{\prime}}{\left(1+\lambda N+\bar{\lambda}^{\prime} N^{\prime}\right)^{2}}+2 s-a c_{R I} \frac{N^{\prime}}{N}\right)^{2}}<0
$$

hence the phenotypic distance decreases with the basal predation rate $d$. Assuming strong female choosiness and predator discrimination $\left(H_{s}\right)$ leads to similar effect (Figure $5(\mathrm{a})$ ). 
When $a$ and $b$ are of order $\varepsilon\left(H_{w}\right)$,

298

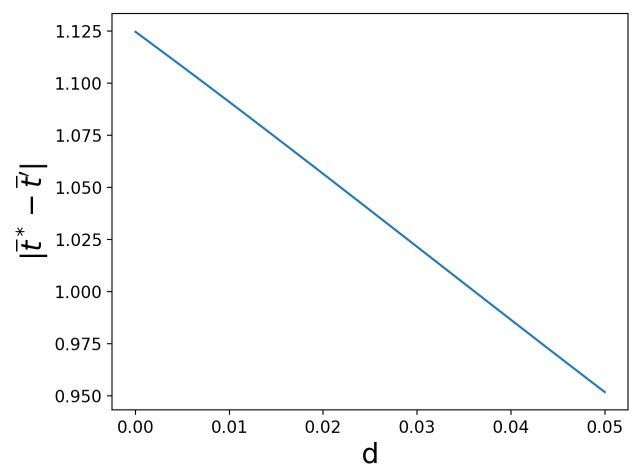

(a)

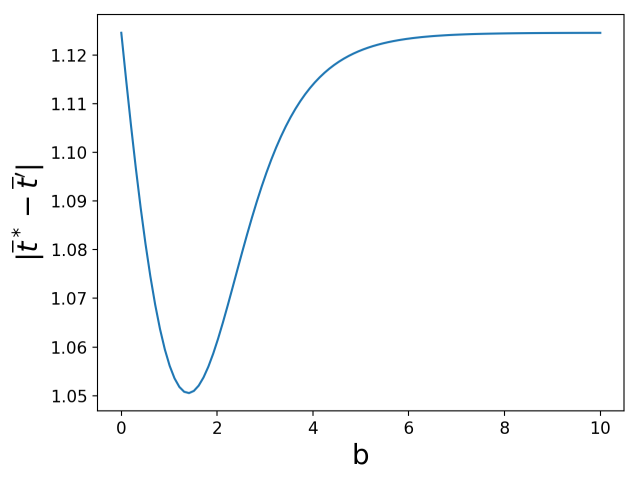

(b)

Figure 5: Influence of the strength of predation $d$ (a) and of predator discrimination $b(\mathrm{~b})$ on the phenotypic distances between the focal species and the local mimicry rings, assuming strong female choosiness and predator discrimination.

The default parameters values are as follows: $a=1, c_{R I}=0.01, b=1, d=0.02, \lambda=0.1$, $\bar{\lambda}^{\prime}=0.1, N=10, N^{\prime}=20, s=0.0025, t_{a}=0, \bar{t}^{\prime}=1$. 


\subsubsection{Effect of the defence level of the focal species}

Without reproductive interference $\left(c_{R I}=0\right)$ and when $a$ and $b$ are of order $\varepsilon\left(H_{w}\right)$, we

2

Therefore, the more defended the focal species is, the greater is the phenotypic distance between the focal species and the local mimicry ring.

Assuming reproductive interference generates cost $\left(c_{R I}>0\right)$, the density of the focal species then modulates the fitness cost incurred by RI. Therefore, we study the effect of the two components of the level of defence of the focal species: the individual defence level $\lambda$ and the density $N$.

$$
\frac{\partial\left|\bar{t}^{*}-\bar{t}^{\prime}\right|}{\partial \lambda}=\frac{2 b d \bar{\lambda}^{\prime} N^{\prime} N}{\left(1+\lambda N+\bar{\lambda}^{\prime} N^{\prime}\right)^{3}} \frac{2 s\left|t_{a}-\bar{t}^{\prime}\right|}{\left(\frac{2 b d \bar{\lambda}^{\prime} N^{\prime}}{\left(1+\lambda N+\bar{\lambda}^{\prime} N^{\prime}\right)^{2}}+2 s-a c_{R I} \frac{N^{\prime}}{N}\right)^{2}}>0
$$

indicates that the individual defence level increases the phenotypic distance. Assuming strong female choosiness and predator discrimination leads to similar effect (Figure 6).

We also have

$$
\frac{\partial\left|\bar{t}^{*}-\bar{t}^{\prime}\right|}{\partial N}=-\frac{1}{N}\left(a c_{R I} \frac{N^{\prime}}{N}-\frac{4 b d(\lambda N)\left(\bar{\lambda}^{\prime} N^{\prime}\right)}{\left(1+\lambda N+\bar{\lambda}^{\prime} N^{\prime}\right)^{3}}\right) \frac{2 s\left|t_{a}-\bar{t}^{\prime}\right|}{\left(\frac{2 b d \bar{\lambda}^{\prime} N^{\prime}}{\left(1+\lambda N+\bar{\lambda}^{\prime} N^{\prime}\right)^{2}}+2 s-a c_{R I} \frac{N^{\prime}}{N}\right)^{2}}
$$

While strong divergence is promoted when the focal species has a low abundance, for larger abundances, the effect of the density on the phenotypic distance between the focal species and the mimicry ring depends on the strength of reproductive interference and predation (Figure 7). When fitness cost due to reproductive interference is great compared to fitness cost due to predation, an increase in the density of the focal species decreases the phenotypic distance, because its reduces the fitness cost due to reproductive interference 


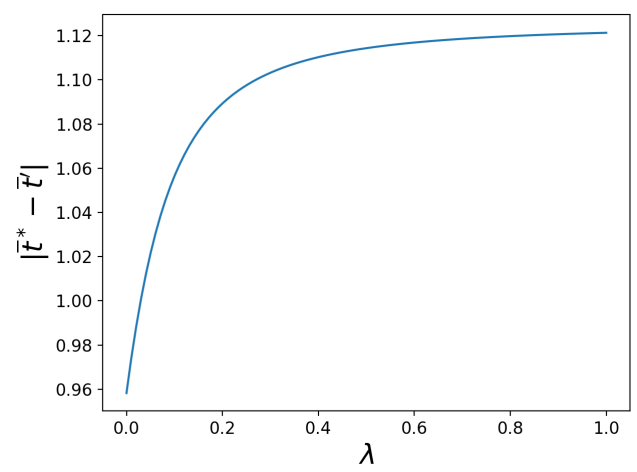

Figure 6: Influence of the individual defence level $\lambda$ on the phenotypic distances between the focal species and the local mimicry rings, assuming strong female choosiness and predator discrimination. The default parameters values are as follows: $a=1, c_{R I}=0.01, b=1, d=0.02, \lambda=0.1, \bar{\lambda}^{\prime}=0.1, N=10, N^{\prime}=20, s=0.0025, t_{a}=0$, $\bar{t}^{\prime}=1$.

(similar effect observed assuming strong female choosiness and predator discrimination $\left(H_{s}\right)$ (Figure $\left.\left.7(\mathrm{a})\right)\right)$. By contrast, when fitness cost due to reproductive interference is low compared to fitness cost due to predation, an increase in the density of the focal species increases the phenotypic distance because the focal species is better defended (similar effect observed assuming strong female choosiness and predator discrimination $\left(H_{s}\right)$ (see right part of Figure $7(\mathrm{~b})))$.

\subsubsection{Effect of selective and developmental constraints promoting the ances-} tral trait value $t_{a}$.

When $a$ and $b$ are of order $\varepsilon$,

$$
\frac{\partial\left|\bar{t}^{*}-\bar{t}^{\prime}\right|}{\partial s}=\frac{2\left|t_{a}-\bar{t}^{\prime}\right|\left(\frac{2 b d \bar{\lambda}^{\prime} N^{\prime}}{\left(1+\lambda N+\bar{\lambda}^{\prime} N^{\prime}\right)^{2}}-a c_{R I} \frac{N^{\prime}}{N}\right)}{\left(\frac{2 b d \bar{\lambda}^{\prime} N^{\prime}}{\left(1+\lambda N+\bar{\lambda}^{\prime} N^{\prime}\right)^{2}}+2 s-a c_{R I} \frac{N^{\prime}}{N}\right)^{2}} .
$$




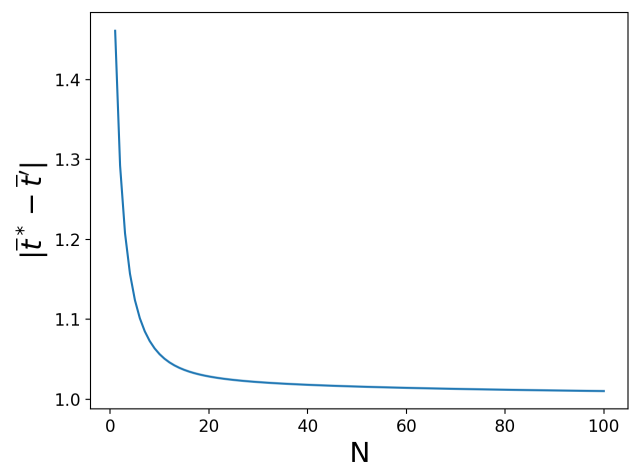

(a)

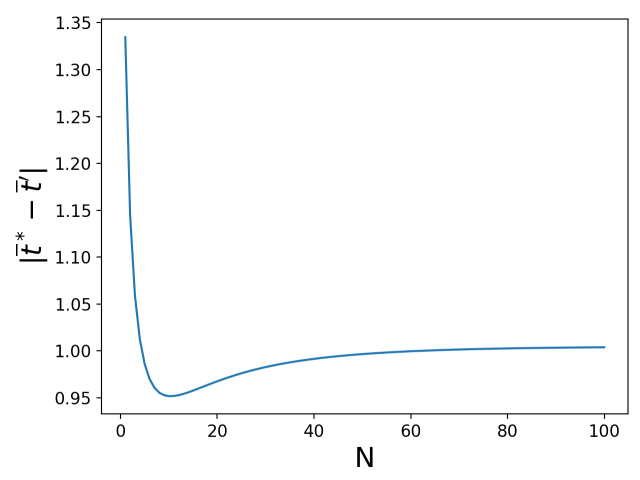

(b)

Figure 7: Influence of the density of the focal species $N$ on the phenotypic distances between the focal species and the local mimicry rings, when (b) predation is low compared to reproductive interference and (b) when predation is high compared to RI, assuming strong female choosiness and predator discrimination. Simulations were run assuming: (a) $d=0.02$ or (b) $d=0.05$. The default parameters values are as follows: $a=1, c_{R I}=0.01, b=1, \lambda=0.1, \bar{\lambda}^{\prime}=0.1, N=10$, $N^{\prime}=20, s=0.0025, t_{a}=0, \bar{t}^{\prime}=1$. 


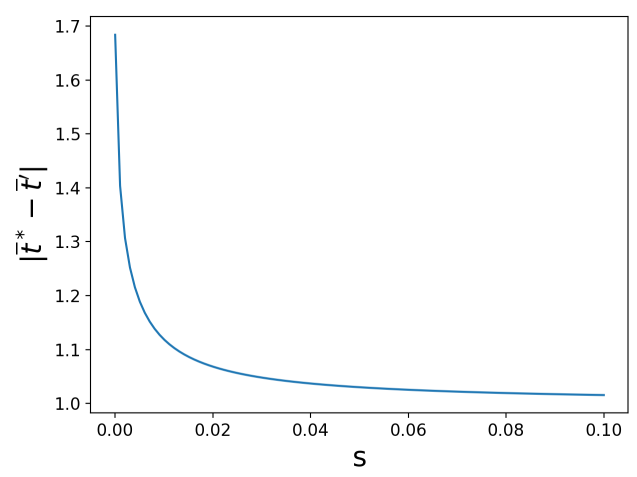

(a)

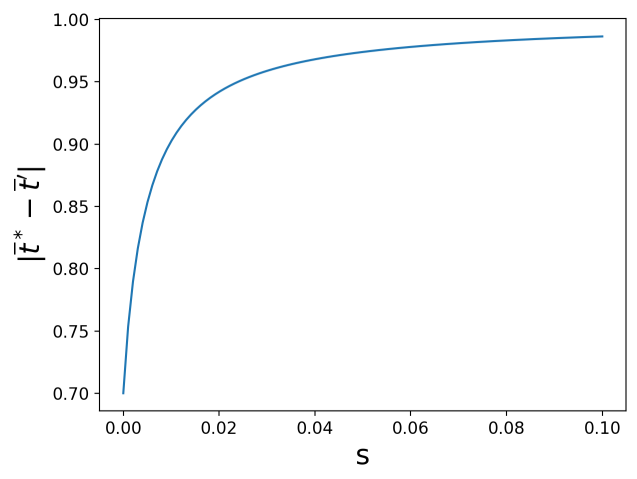

(b)

Figure 8: Influence of the strength of selective constraints $s$ on the phenotypic distances between the focal species and the local mimicry rings, when (b) predation is low compared to reproductive interference and (b) when predation is great compared to RI, assuming strong female choosiness and predator discrimination. Simulations were run assuming: (a) $d=0.02$ or (b) $d=0.05$. The default parameters values are as follows: $a=1, c_{R I}=0.01, b=1, \lambda=0.1, \bar{\lambda}^{\prime}=0.1, N=10$, $N^{\prime}=20, s=0.0025, t_{a}=0, \bar{t}^{\prime}=1$.

\subsubsection{Effect of the mimetic community.}

Finally, we investigate the impact of the composition of the local mimicry ring (density $N^{\prime}$ and defense level $\left.\lambda^{\prime}\right)$ on trait divergence. When $a$ and $b$ are of order $\varepsilon\left(H_{w}\right)$, we have:

$$
\frac{\partial\left|\bar{t}^{*}-\bar{t}^{\prime}\right|}{\partial \bar{\lambda}^{\prime}}=\frac{2 b d N^{\prime}\left(\bar{\lambda}^{\prime} N^{\prime}-1-\lambda N\right)}{\left(1+\lambda N+\bar{\lambda}^{\prime} N^{\prime}\right)^{3}} \frac{2 s\left|t_{a}-\bar{t}^{\prime}\right|}{\left(\frac{2 b d \bar{\lambda}^{\prime} N^{\prime}}{\left(1+\lambda N+\bar{\lambda}^{\prime} N^{\prime}\right)^{2}}+2 s-a c_{R I} \frac{N^{\prime}}{N}\right)^{2}},
$$

and

$354 \quad \frac{\partial\left|\bar{t}^{*}-\bar{t}^{\prime}\right|}{\partial N^{\prime}}=\left(\frac{2 b d \bar{\lambda}^{\prime}\left(\bar{\lambda}^{\prime} N^{\prime}-1-\lambda N\right)}{\left(1+\lambda N+\bar{\lambda}^{\prime} N^{\prime}\right)^{3}}+\frac{a c_{R I}}{N}\right) \frac{2 s\left|t_{a}-\bar{t}^{\prime}\right|}{\left(\frac{2 b d \bar{\lambda}^{\prime} N^{\prime}}{\left(1+\lambda N+\bar{\lambda}^{\prime} N^{\prime}\right)^{2}}+2 s-a c_{R I} \frac{N^{\prime}}{N}\right)^{2}}$. 
Thus, when the defence level of the local mimicry ring $\left(\bar{\lambda}^{\prime} N^{\prime}\right)$ is lower than the defence level of the focal species plus one $\left(\bar{\lambda}^{\prime} N^{\prime}<1+\lambda N\right)$, an increase in the defence level of individuals of the local mimicry ring promotes phenotypic similarity between the focal species and the local mimicry ring (similar effect observed assuming strong but moderate female choosiness and predator discrimination $\left(H_{s}\right)$ (Figure 9(a))). Indeed, an increase of $\bar{\lambda}^{\prime} N^{\prime}$ increases the advantage of looking similar to individuals of the local mimicry ring. More surprisingly, when the defence level of the local mimicry ring is greater $\left(\bar{\lambda}^{\prime} N^{\prime}>1+\lambda N\right)$, the phenotypic distance increases with $\bar{\lambda}^{\prime} N^{\prime}$ (similar effect observed assuming strong but moderate female choosiness and predator discrimination (Figure $9(\mathrm{a})))$. Under weak $(b=O(\varepsilon))$ or strong but moderate $(e . g . b=1)$ predator discrimination, predators tend to generalize mimetic trait. Then individuals of the focal species gain at least a little protection from the local mimicry ring, even when displaying a imperfectly mimetic trait. When the level of protection of the mimicry ring is high, the fitness costs linked to predation is low compared to the reproductive interference and decreases with the defence level of individuals of the local mimicry ring. This decrease in selection pressure generated by predation decreases the phenotype similarity between the focal species and the local mimicry ring. When predator discrimination is strong and high (e.g. $b=10)$, there is no generalization of imperfect mimics by predators, this effect is no longer observed (see Figure 9(b)).

The density $N^{\prime}$ and the individual defence level of the local mimicry ring $\lambda^{\prime}$ have the same effect on the overall defence level of the local mimicry ring (function of the parameter $\bar{\lambda}^{\prime} N^{\prime}$ ), and therefore have a similar effect on the phenotypic distance. However, the density of the local mimicry ring $N^{\prime}$ also plays a role on the fitness costs generated by RI. When the strength of reproductive interference is high as compared to predation, the phenotypic distance increases with the density of the local mimicry ring $\left(N^{\prime}\right)$, because 


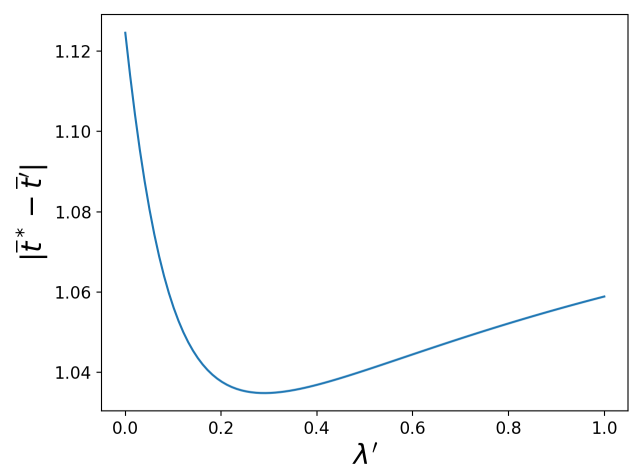

(a)

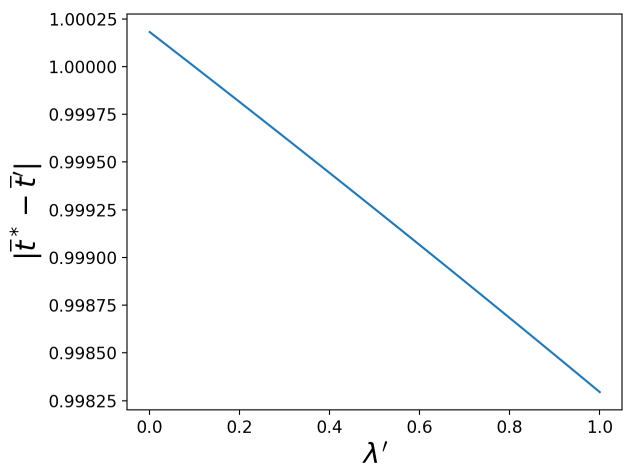

(b)

Figure 9: Influence of the mean defence level of individuals of the local mimicry ring $\bar{\lambda}^{\prime}$ on the phenotypic distances between the focal species and the local mimicry rings for $(\mathbf{a})$ moderate $(b=1)$ and $(\mathbf{b})$ high $(b=10)$ predator discrimination, assuming strong female choosiness and predator discrimination (i.e. $a=O(1)$ and $b=O(1))$. Simulations were run assuming: (a) $a=1$ and (b) $a=10$. The default parameters values are as follows: $a=1, c_{R I}=0.01, d=0.02, \lambda=0.1, \bar{\lambda}^{\prime}=0.1$, $N=10, N^{\prime}=20, s=0.0025, t_{a}=0, \bar{t}^{\prime}=1$. 


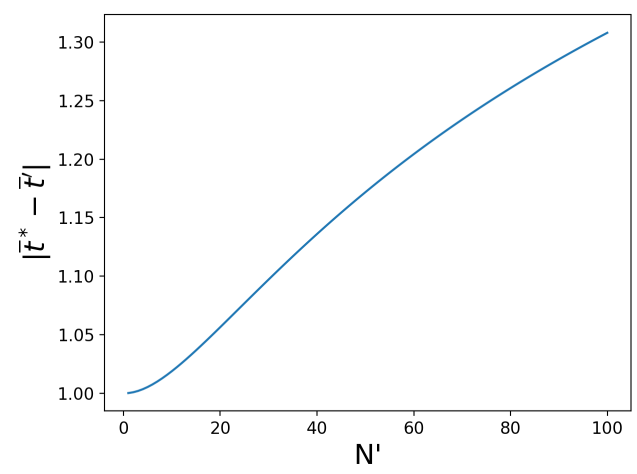

(a)

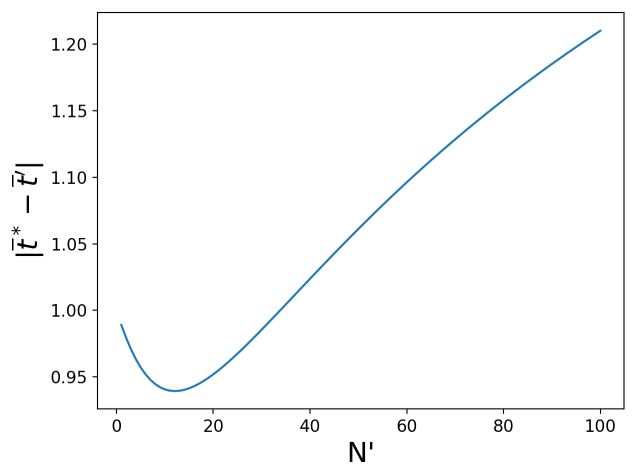

(b)

Figure 10: Influence of the density of the local mimicry ring $N^{\prime}$ on the phenotypic distances between the focal species and the local mimicry rings, when (b) predation is low compared to reproductive interference and (b) when predation is high compared to RI, assuming strong female choosiness and predator discrimination. Simulations were run assuming: (a) $d=0.02$ or (b) $d=0.05$. The default parameters values are as follows: $a=1, c_{R I}=0.01, b=1, \lambda=0.1, \bar{\lambda}^{\prime}=0.1, N=10$, $N^{\prime}=20, s=0.0025, t_{a}=0, \bar{t}^{\prime}=1$.

\section{Model 2: Evolution of mimicry between two interacting} species 


\begin{tabular}{ll}
\hline Abbreviation & Description \\
\hline $\bar{t}_{i} / \bar{p}_{i}$ & Mean trait/preference value in the species $i$ \\
$G_{t_{i}} / G_{p_{i}}$ & Genetic variance of trait $t_{i} /$ preference $p_{i}$ \\
$C_{t_{i} p_{i}}$ & Genetic covariance between trait $t_{i}$ and preference $p_{i}$ \\
$\beta_{t_{i}} / \beta_{p_{i}}$ & Selection coefficient on trait $t_{i} /$ preference $p_{i}$ \\
$a_{i}$ & Female choosiness in species $i$ \\
$s_{i}$ & Strength of developmental constraints in species $i$ \\
$t_{a i}$ & Ancestral trait favored by developmental constraints in species $i$ \\
$d$ & Basal predation rate \\
$b$ & Predator discrimination \\
$\lambda_{i}$ & Unpalatability coefficient of individuals of the species $i$ \\
$N_{i}$ & Density of the species $i$ \\
$c_{R I}$ & Strength of reproductive interference \\
\hline
\end{tabular}

Table 1: Description of variables and parameters used in the model with two species. The subscript $i \in\{1,2\}$ denotes the species.

parameter of the interspecific phenotypic distance.

\subsection{Parameters of the model}

The parameters used in the model are summed up in Table 1.

\subsection{Selection coefficients}

In this section we detail how we obtain the expressions for the selection vectors. 
For $i \in\{1,2\}$, the selection coefficients are defined by:

400

402

418

$$
\begin{aligned}
& \beta_{t_{i}}:=\frac{\partial}{\partial t_{m}} \ln W^{i}\left(t_{m}, t_{f}, p_{f}\right)+\left.\frac{\partial}{\partial t_{f}} \ln W^{i}\left(t_{m}, t_{f}, p_{f}\right)\right|_{\left(t_{m}, t_{f}, p_{f}\right)=(\bar{t}, \bar{t}, \bar{p})}, \\
& \beta_{p_{i}}:=\left.\frac{\partial}{\partial p_{f}} \ln W^{i}\left(t_{m}, t_{f}, p_{f}\right)\right|_{\left(t_{m}, t_{f}, p_{f}\right)=(\bar{t}, \bar{t}, \bar{p})},
\end{aligned}
$$

where $W^{i}$ are the fitness of a mated pair of a male with trait $t_{m}$ and a female with trait $t_{f}$ and preference $p_{f}$ of the species $i$, and write

$$
W^{i}\left(t_{m}, t_{f}, p_{f}\right)=W_{n s}^{i}\left(t_{m}\right) W_{r}^{i}\left(t_{m}, p_{f}\right) W_{n s}^{i}\left(t_{f}\right) W_{R I}^{i}\left(p_{f}\right)
$$

Here $W_{n s}^{i}$ is the impact of natural selection on fitness and $W_{R I}^{i}$ is the impact reproductive interference on fitness of individuals of species $i$.

\subsubsection{Computation of $\beta_{t_{1}}$ and $\beta_{t_{2}}$}

Similarly as the case of one population in a mimicry ring studied previously (recall the definition of $W_{\text {pred }}$ in (11)), the impact of predation on an individual of trait $t$ is:

$$
\begin{aligned}
\frac{\partial}{\partial t_{1}} \ln \left(W_{\text {pred }}^{2}\left(t_{1}\right)\right)= & -2 b\left(\left(t_{1}-\bar{t}_{1}\right) \lambda_{1} \exp \left[-b\left(t_{1}-\bar{t}_{1}\right)^{2}\right] N_{1}+\left(t_{2}-\bar{t}_{2}\right) \bar{\lambda}_{2} \exp \left[-b\left(t_{2}-\bar{t}_{2}\right)^{2}\right] N_{2}\right) \\
& \times\left(\frac{1}{1+\lambda_{1} N_{1} \exp \left[-b\left(t_{2}-\bar{t}_{1}\right)^{2}\right]+\lambda_{2} \exp \left[-b\left(t_{2}-\bar{t}_{2}\right)^{2}\right] N_{2}-d}\right. \\
& \left.-\frac{1}{1+\lambda_{1} N_{1} \exp \left[-b\left(t_{2}-\bar{t}_{1}\right)^{2}\right]+\lambda_{2} \exp \left[-b\left(t_{2}-\bar{t}_{2}\right)^{2}\right] N_{2}}\right)
\end{aligned}
$$

By symmetry we obtain the expression of $\frac{\partial}{\partial t_{2}} \ln \left(W_{\text {pred }}^{2}\left(t_{2} \mid \bar{t}_{1}, \bar{t}_{2}\right)\right)$ by exchanging 1 and 2. When $b$ is of order $\varepsilon\left(H_{w}\right)$, similar computations as in the case of one population in a mimicry ring yield:

$$
\begin{aligned}
& \beta_{t_{1}}=-2 a_{1}\left(\bar{t}_{1}-\bar{p}_{1}\right)-\frac{4 b d\left(\bar{t}_{1}-\bar{t}_{2}\right) \lambda_{2} N_{2}}{\left(1+\lambda_{1} N_{1}+\lambda_{2} N_{2}\right)^{2}}-4 s_{1}\left(\bar{t}_{1}-t_{a 1}\right)+O\left(\varepsilon^{3}\right), \\
& \beta_{t_{2}}=-2 a_{2}\left(\bar{t}_{2}-\bar{p}_{2}\right)-\frac{4 b d\left(\bar{t}_{2}-\bar{t}_{1}\right) \lambda_{1} N_{1}}{\left(1+\lambda_{1} N_{1}+\lambda_{2} N_{2}\right)^{2}}-4 s_{2}\left(\bar{t}_{2}-t_{a 2}\right)+O\left(\varepsilon^{3}\right) .
\end{aligned}
$$




\subsubsection{Computation of $\beta_{p_{1}}$ and $\beta_{p_{2}}$}

422

436

438

440

442

Let $W_{R I}^{i}(p)$ indicate the impact of reproductive interference on a female of species $i$ with preference $p$. If we denote by $j$ the complement of $i$ in $\{1,2\}$, we get:

$$
W_{R I}^{i}\left(p_{i}\right)=\frac{\exp \left[-a_{i}\left(p_{i}-\bar{t}_{i}\right)^{2}\right] N_{i}}{\left.\exp \left[-a_{i}\left(p_{i}-\bar{t}_{i}\right)^{2}\right] N_{i}+c_{R I} \exp \left[-a_{j}\left(p_{i}-\bar{t}_{j}\right)^{2}\right)\right] N_{j}} .
$$

In the same way as in the previous model we thus get, when we assume that $a_{1}, a_{2}$ and $c_{R I}$ are of order $\varepsilon\left(H_{w}\right)$,

\subsection{Quasi equilibria}

We search mean values of traits and preferences at equilibrium $\bar{t}_{1}^{*}, \bar{p}_{1}^{*}, \bar{t}_{2}^{*}$ and $\bar{p}_{2}^{*}$ such as the leading terms of $\Delta \bar{t}_{1}^{*}, \Delta \bar{p}_{1}^{*}, \Delta \bar{t}_{2}^{*}$ and $\Delta \bar{p}_{2}^{*}$ are null, or equivalently, $\tilde{\beta}_{t_{1}}=\tilde{\beta}_{p_{1}}=\tilde{\beta}_{t_{2}}=\tilde{\beta}_{p_{2}}=0$ with

$$
\begin{aligned}
& \beta_{p_{1}}=-2 a_{1}\left(\bar{p}_{1}-\bar{t}_{1}\right)+2 c_{R I} a_{1}\left(\bar{t}_{1}-\bar{t}_{2}\right) \frac{N_{2}}{N_{1}}+O\left(\varepsilon^{3}\right), \\
& \beta_{p_{2}}=-2 a_{2}\left(\bar{p}_{2}-\bar{t}_{2}\right)+2 c_{R I} a_{2}\left(\bar{t}_{2}-\bar{t}_{1}\right) \frac{N_{1}}{N_{2}}+O\left(\varepsilon^{3}\right) .
\end{aligned}
$$

$$
\begin{aligned}
& \tilde{\beta}_{t_{1}}=-2 a_{1}\left(\bar{t}_{1}^{*}-\bar{p}_{1}^{*}\right)-\frac{4 b d\left(\bar{t}_{1}^{*}-\bar{t}_{2}^{*}\right) \lambda_{2} N_{2}}{\left(1+\lambda_{1} N_{1}+\lambda_{2} N_{2}\right)^{2}}-4 s_{1}\left(\bar{t}_{1}^{*}-t_{a 1}\right), \\
& \tilde{\beta}_{p_{1}}=-2 a_{1}\left(\bar{p}_{1}^{*}-\bar{t}_{1}^{*}\right)+2 c_{R I} a_{1}\left(\bar{t}_{1}^{*}-\bar{t}_{2}^{*}\right) \frac{N_{2}}{N_{1}} \\
& \tilde{\beta}_{t_{2}}=-2 a_{2}\left(\bar{t}_{2}^{*}-\bar{p}_{2}^{*}\right)-\frac{4 b d\left(\bar{t}_{2}^{*}-\bar{t}_{1}^{*}\right) \lambda_{1} N_{1}}{\left(1+\lambda_{1} N_{1}+\lambda_{2} N_{2}\right)^{2}}-4 s_{2}\left(\bar{t}_{2}^{*}-t_{a 2}\right), \\
& \tilde{\beta}_{p_{2}}=-2 a_{2}\left(\bar{p}_{2}^{*}-\bar{t}_{2}^{*}\right)+2 c_{R I} a_{2}\left(\bar{t}_{2}^{*}-\bar{t}_{1}^{*}\right) \frac{N_{1}}{N_{2}}
\end{aligned}
$$

Lemma 3. Let us introduce for $i \neq j \in\{1,2\}^{2}$,

$$
\begin{aligned}
A_{i} & =\frac{2 b d \lambda_{j} N_{j}-a_{i} c_{R I} \frac{N_{j}}{N_{i}}\left(1+\lambda_{1} N_{1}+\lambda_{2} N_{2}\right)^{2}}{2 b d \lambda_{j} N_{j}+\left(2 s_{i}-a_{i} c_{R I} \frac{N_{j}}{N_{i}}\right)\left(1+\lambda_{1} N_{1}+\lambda_{2} N_{2}\right)^{2}}, \\
B_{i} & =\frac{2 s_{i} t_{a i}\left(1+\lambda_{1} N_{1}+\lambda_{2} N_{2}\right)^{2}}{2 b d \lambda_{j} N_{j}+\left(2 s_{i}-a_{i} c_{R I} \frac{N_{j}}{N_{i}}\right)\left(1+\lambda_{1} N_{1}+\lambda_{2} N_{2}\right)^{2}} .
\end{aligned}
$$


444

If $2 b d \lambda_{j} N_{j}+\left(2 s_{i}-a_{i} c_{R I}\left(N_{j} / N_{i}\right)\right)\left(1+\lambda_{1} N_{1}+\lambda_{2} N_{2}\right)^{2} \neq 0$ for $i \in\{1,2\}$ and if $A_{1} A_{2} \neq 1$ there is a unique quasi equilibrium point given for $i \in\{1,2\}$ by

$$
\bar{t}_{i}^{*}=\frac{B_{i}+A_{i} B_{j}}{1-A_{1} A_{2}}, \quad \bar{p}_{i}^{*}=\bar{t}_{i}^{*}+c_{R I}\left(\bar{t}_{i}^{*}-\bar{t}_{j}^{*}\right) \frac{N_{j}}{N_{i}},
$$

Remark. We do not give the quasi equilibrium traits and preferences when one of the following conditions is verified:

- $2 b d \lambda_{2} N_{2}+\left(2 s_{1}-a_{1} c_{R I}\left(N_{2} / N_{1}\right)\right)\left(1+\lambda_{1} N_{1}+\lambda_{2} N_{2}\right)^{2}=0$

- $2 b d \lambda_{1} N_{1}+\left(2 s_{2}-a_{2} c_{R I}\left(N_{1} / N_{2}\right)\right)\left(1+\lambda_{1} N_{1}+\lambda_{2} N_{2}\right)^{2}=0$,

- $A_{1} A_{2}=1$.

Indeed investigating those cases leads to a long enumeration of subcases with different quasi equilibrium points, and those cases are negligible compared with all the possible combinations of parameters. We thus choose to not give this enumeration.

Proof. Let $2 b d \lambda_{j} N_{j}+\left(2 s_{i}-a_{i} c_{R I}\left(N_{j} / N_{i}\right)\right)\left(1+\lambda_{1} N_{1}+\lambda_{2} N_{2}\right)^{2} \neq 0$ for $i \neq j \in\{1,2\}^{2}$. Using the results of the first model (see Appendix 1.3) we have

$$
\bar{t}_{1}^{*}=\frac{\frac{2 b d \lambda_{2} N_{2}}{\left(1+\lambda_{1} N_{1}+\lambda_{2} N_{2}\right)^{2}} \bar{t}_{2}^{*}+2 s_{1} t_{a 1}-a_{1} c_{R I} \frac{N 2}{N 1} \bar{t}_{2}^{*}}{\frac{2 b d \lambda_{2} N_{2}}{\left(1+\lambda_{1} N_{1}+\lambda_{2} N_{2}\right)^{2}}+2 s_{1}-a_{1} c_{R I} \frac{N_{2}}{N_{1}}},
$$

and

$$
\bar{t}_{2}^{*}=\frac{\frac{2 b d \lambda_{1} N_{1}}{\left(1+\lambda_{1} N_{1}+\lambda_{2} N_{2}\right)^{2}} \bar{t}_{1}^{*}+2 s_{2} t_{a 2}-a_{2} c_{R I} \frac{N 1}{N 2} \bar{t}_{1}^{*}}{\frac{2 b d \lambda_{1} N_{1}}{\left(1+\lambda_{1} N_{1}+\lambda_{2} N_{2}\right)^{2}}+2 s_{2}-a_{2} c_{R I} \frac{N_{1}}{N_{2}}},
$$

which can be rewritten

$$
\bar{t}_{1}^{*}=A_{1} \bar{t}_{2}^{*}+B_{1}, \quad \bar{t}_{2}^{*}=A_{2} \bar{t}_{1}^{*}+B_{2},
$$

where $\left(A_{i}, B_{i}\right), i \in\{1,2\}$ have been defined in A34 - A35. 


\subsection{Fast and slow dynamics.}

In this section we study when trait and preference in the both species converge towards

1. If $C_{1}>0, C_{2}>0$ and $C_{1} C_{2}\left(1-A_{1} A_{2}\right)-\frac{1}{4}\left(A_{1} C_{1}-A_{2} C_{2}\right)^{2}>0$ the quantities to analytically determine the convergence or the divergence of traits and preferences. To infer such information we simulate the dynamic of traits and preferences considering the leading term of the selection coefficients. 
Proof. As previously, we can decompose the dynamics into two steps. In the first one the leading order terms of the selection coefficient are the terms describing sexual selection and cost of choosiness. Using computations similar to the ones in the previous model we can approximate $\Delta\left(\bar{t}_{i}-\bar{p}_{i}\right)^{2}$ for $i \in\{1,2\}$ by

$$
\Delta\left(\bar{t}_{i}-\bar{p}_{i}\right)^{2}=-\left(\bar{t}_{i}-\bar{p}_{i}\right)\left(2 a_{i}\left(G_{t_{i}}+G_{p_{i}}\right)\left(\bar{t}_{i}-\bar{p}_{i}\right)+O\left(\varepsilon^{2}\right)\right)+O\left(\varepsilon^{3}\right) .
$$

Using the same reasoning that in the previous model we may prove that above a certain number of generations $t_{1}-p_{1}$ and $t_{2}-p_{2}$ are of order $\varepsilon$.

We now look at the variation of

$$
\mathcal{A}:=\frac{\left(\bar{t}_{1}-\bar{t}_{1}^{*}\right)^{2}}{G_{t_{1}}}+\frac{\left(\bar{t}_{2}-\bar{t}_{2}^{*}\right)^{2}}{G_{t_{2}}}+\frac{\left(\bar{p}_{1}-\bar{p}_{1}^{*}\right)^{2}}{G_{p_{1}}}+\frac{\left(\bar{p}_{2}-\bar{p}_{2}^{*}\right)^{2}}{G_{p_{2}}}
$$

with $\bar{t}_{1}^{*}, \bar{t}_{2}^{*}, \bar{p}_{1}^{*}$ and $\bar{p}_{2}^{*}$ defined by A36.

By definition,

When $\bar{t}_{1}-\bar{p}_{1}$ and $\bar{t}_{2}-\bar{p}_{2}$ are of order $\varepsilon, \Delta \bar{t}_{1}, \Delta \bar{t}_{2}, \Delta \bar{p}_{1}$ and $\Delta \bar{p}_{2}$ are of order $\varepsilon^{2}$. We then neglect the terms proportional to $\left(\Delta \bar{t}_{1}\right)^{2},\left(\Delta \bar{t}_{2}\right)^{2},\left(\Delta \bar{p}_{1}\right)^{2}$ or $\left(\Delta \bar{p}_{2}\right)^{2}$ :

$$
\Delta \mathcal{A}=\frac{2 \Delta \bar{t}_{1}\left(\bar{t}_{1}-\bar{t}_{1}^{*}\right)}{G_{t_{1}}}+\frac{2 \Delta \bar{t}_{2}\left(\bar{t}_{2}-\bar{t}_{2}^{*}\right)}{G_{t_{2}}}+\frac{2 \Delta \bar{p}_{1}\left(\bar{p}_{1}-\bar{p}_{1}^{*}\right)}{G_{p_{1}}}+\frac{2 \Delta \bar{p}_{2}\left(\bar{p}_{2}-\bar{p}_{2}^{*}\right)}{G_{p_{2}}}+O\left(\varepsilon^{4}\right)
$$

By replacing the terms $\Delta \bar{t}_{1}, \Delta \bar{t}_{2}, \Delta \bar{p}_{1}$ and $\Delta \bar{p}_{2}$ using (12) and simplifying, we obtain:

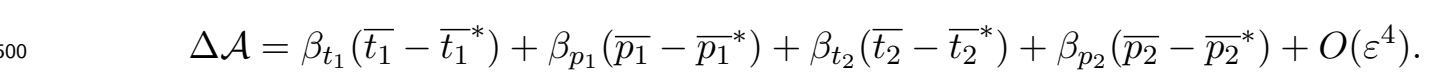

Using that for $i \in\{1,2\}, \bar{t}_{i}-\bar{p}_{i}$ and $\bar{t}_{i}^{*}-\bar{p}_{i}^{*}$ are of order $\varepsilon$, we obtain similarly as in A20

$$
{ }_{502} \Delta \mathcal{A}=\left(\beta_{t_{1}}+\beta_{p_{1}}\right)\left(\frac{\bar{t}_{1}-\bar{t}_{1}^{*}}{2}+\frac{\bar{p}_{1}-\bar{p}_{1}^{*}}{2}\right)+\left(\beta_{t_{2}}+\beta_{p_{2}}\right)\left(\frac{\bar{t}_{2}-\bar{t}_{2}^{*}}{2}+\frac{\bar{p}_{2}-\bar{p}_{2}^{*}}{2}\right)+O\left(\varepsilon^{3}\right) .
$$


Using (A26) and A28) and simplifying, we obtain:

504

506

508

510

$$
\beta_{t_{1}}+\beta_{p_{1}}=-\frac{4 b d\left(\bar{t}_{1}-\bar{t}_{2}\right) \lambda_{2} N_{2}}{\left(1+\lambda_{1} N_{1}+\lambda_{2} N_{2}\right)^{2}}-4 s_{1}\left(\bar{t}_{1}-t_{a 1}\right)+2 c_{R I} a_{1}\left(\bar{t}_{1}-\bar{t}_{2}\right) \frac{N_{2}}{N_{1}}+O\left(\varepsilon^{3}\right) .
$$

By substracting $-\frac{4 b d\left(\bar{t}_{1}^{*}-\bar{t}_{2}^{*}\right) \lambda_{2} N_{2}}{\left(1+\lambda_{1} N_{1}+\lambda_{2} N_{2}\right)^{2}}-4 s_{1}\left(\bar{t}_{1}^{*}-t_{a 1}\right)+2 c_{R I} a_{1}\left(\bar{t}_{1}^{*}-\bar{t}_{2}^{*}\right) \frac{N_{2}}{N_{1}}$ which is equal to zero (because $\left.\beta_{t_{1}}\right|_{\left(t_{1}, t_{2}, p_{1}, p_{2}\right)=\left(\bar{t}_{1}^{*}, \bar{t}_{2}^{*}, \bar{p}_{1}^{*}, \bar{p}_{2}^{*}\right)}=0$ and $\left.\beta_{p_{1}}\right|_{\left(t_{1}, t_{2}, p_{1}, p_{2}\right)=\left(\bar{t}_{1}^{*}, \bar{t}_{2}^{*}, \bar{p}_{1}^{*}, \bar{p}_{2}^{*}\right)}=0$, we get:

$$
\begin{aligned}
\left(\beta_{t_{1}}+\beta_{p_{1}}\right) & =\left(-\frac{4 b d \lambda_{2} N_{2}}{\left(1+\lambda_{1} N_{1}+\lambda_{2} N_{2}\right)^{2}}-4 s_{1}+2 c_{R I} a_{1} \frac{N_{2}}{N_{1}}\right)\left(\bar{t}_{1}-\bar{t}_{1}^{*}\right) \\
& -\left(-\frac{4 b d \lambda_{2} N_{2}}{\left(1+\lambda_{1} N_{1}+\lambda_{2} N_{2}\right)^{2}}+2 c_{R I} a_{1} \frac{N_{2}}{N_{1}}\right)\left(\bar{t}_{2}-\bar{t}_{2}^{*}\right)+O\left(\varepsilon^{3}\right) \\
& =-C_{1}\left(\bar{t}_{1}-\bar{t}_{1}^{*}\right)+A_{1} C_{1}\left(\bar{t}_{2}-\bar{t}_{2}^{*}\right)+O\left(\varepsilon^{3}\right) .
\end{aligned}
$$

Because for $i \in\{1,2\}, \bar{t}_{i}-\bar{t}_{i}^{*}=\bar{p}_{i}-\bar{p}_{i}^{*}+\bar{t}_{i}-\bar{p}_{i}+\bar{p}_{i}^{*}-\bar{t}_{i}^{*}=\bar{p}_{i}-\bar{p}_{i}^{*}+O(\varepsilon)$ we also have

$$
\left(\beta_{t_{1}}+\beta_{p_{1}}\right)=-C_{1}\left(\bar{p}_{1}-\bar{p}_{1}^{*}\right)+A_{1} C_{1}\left(\bar{p}_{2}-\bar{p}_{2}^{*}\right)+O\left(\varepsilon^{3}\right)
$$

We thus get

and

$$
\left(\beta_{t_{1}}+\beta_{p_{1}}\right)\left(\bar{t}_{1}-\bar{t}_{1}^{*}\right)=-C_{1}\left(\bar{t}_{1}-\bar{t}_{1}^{*}\right)^{2}+A_{1} C_{1}\left(\bar{t}_{1}-\bar{t}_{1}^{*}\right)\left(\bar{t}_{2}-\bar{t}_{2}^{*}\right)+O\left(\varepsilon^{3}\right)
$$

Hence using (A37), the symmetry of the model between populations 1 and 2, and that for $i \in\{1,2\}, \bar{t}_{i}-\bar{p}_{i}$ and $\bar{t}_{i}^{*}-\bar{p}_{i}^{*}$ are of order $\varepsilon$, we obtain:

$$
\begin{aligned}
2 \Delta \mathcal{A}= & -C_{1}\left(\bar{t}_{1}-\bar{t}_{1}^{*}\right)^{2}+\left(A_{1} C_{1}+A_{2} C_{2}\right)\left(\bar{t}_{1}-\bar{t}_{1}^{*}\right)\left(\bar{t}_{2}-\bar{t}_{2}^{*}\right)-C_{2}\left(\bar{t}_{2}-\bar{t}_{2}^{*}\right)^{2} \\
& -C_{1}\left(\bar{p}_{1}-\bar{p}_{1}^{*}\right)^{2}+\left(A_{1} C_{1}+A_{2} C_{2}\right)\left(\bar{p}_{1}-\bar{p}_{1}^{*}\right)\left(\bar{p}_{2}-\bar{p}_{2}^{*}\right)-C_{2}\left(\bar{p}_{2}-\bar{p}_{2}^{*}\right)^{2},
\end{aligned}
$$

with can be rewritten in matrix form

$$
2 \Delta \mathcal{A}=-T^{t} M T-P^{t} M P+O\left(\varepsilon^{3}\right),
$$


with

530

$$
M=\left(\begin{array}{cc}
C_{1} & -\frac{A_{1} C_{1}+A_{2} C_{2}}{2} \\
-\frac{A_{1} C_{1}+A_{2} C_{2}}{2} & C_{2}
\end{array}\right), \quad T=\left(\begin{array}{c}
\bar{t}_{1}-\bar{t}_{1}^{*} \\
\bar{t}_{2}-\bar{t}_{2}^{*}
\end{array}\right), \quad P=\left(\begin{array}{l}
\bar{p}_{1}-\bar{p}_{1}^{*} \\
\bar{p}_{2}-\bar{p}_{2}^{*}
\end{array}\right),
$$

and $T^{t}$ and $P^{t}$ being the transposed of $T$ and $P$ respectively.

First case: $C_{1}>0, C_{2}>0$ and $C_{1} C_{2}\left(1-A_{1} A_{2}\right)-\frac{1}{4}\left(A_{1} C_{1}-A_{2} C_{2}\right)^{2}>0$.

According to the Sylvester's criterion the matrix $M$ is positive-definite. Using computations similar to the ones derived to prove (A15), we may prove that after a number of generations large enough, $\bar{t}_{1}-\bar{t}_{1}^{*}, \bar{t}_{2}-\bar{t}_{2}^{*}, \bar{p}_{1}-\bar{p}_{1}^{*}$ and $\bar{p}_{2}-\bar{p}_{2}^{*}$ become of order $\varepsilon^{1 / 2}$.

Second case: $C_{1}<0, C_{2}<0$ and $C_{1} C_{2}\left(1-A_{1} A_{2}\right)-\frac{1}{4}\left(A_{1} C_{1}-A_{2} C_{2}\right)^{2}>0$.

According to the Sylvester's criterion the matrix $-M$ is positive-definite. Therefore $\bar{t}_{1}, \bar{t}_{2}, \bar{p}_{1}$ and $\bar{p}_{2}$ become very large.

\subsection{Effect of different parameters on the level of trait divergence $\left|\bar{t}_{1}^{*}-\bar{t}_{2}^{*}\right|$ assuming weak or strong female choosiness and predator discrimina- tion}

We want to verify that the different parameters have similar effect on phenotypic distance between species in this model than in the first. To this aim we plot the effect of different parameters on the level of trait divergence assuming whether $\left(H_{w}\right)$ (using the analytical approximation when possible or numerical simulations) or $\left(H_{s}\right)$ (using numerical simulations). The following plots confirm that the parameters have a similar effect on the phenotypic distance. 


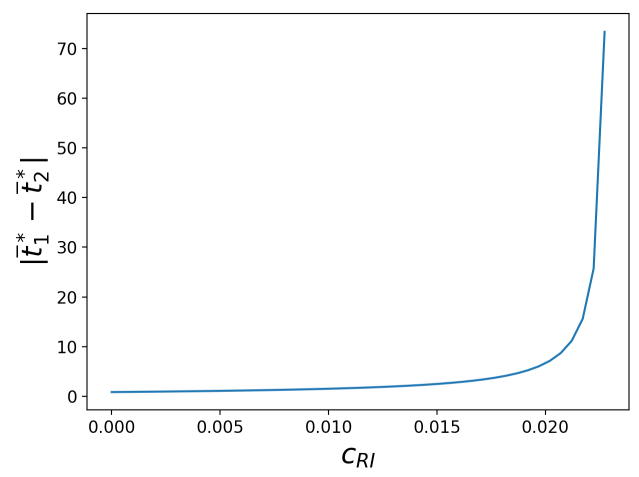

(a)

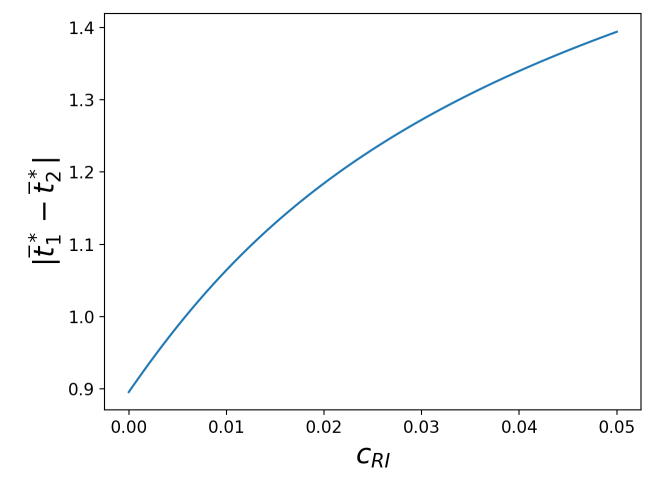

(b)

Figure 11: Influence of the strength of reproductive interference $c_{R I}$ on the phenotypic distances between the focal species and the local mimicry rings, assuming (a) weak or (b) strong female choosiness and predator discrimination. Simulations were run assuming (a) $a_{1}=a_{2}=b=0.01$ or (b) $a_{1}=a_{2}=b=1$. The default parameters values are as follows: $d=0.02, \lambda_{1}=0.1, \lambda_{2}=0.1, N_{1}=10, N_{2}=20$, $s_{1}=0.0000025, s_{2}=0.0000025, t_{a 1}=0, t_{a 2}=1$. 


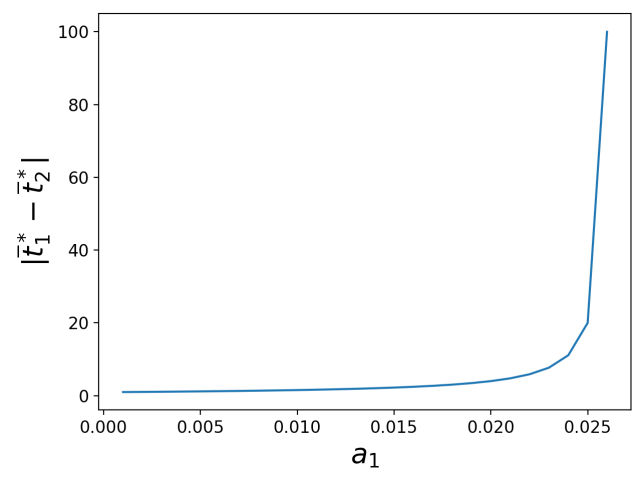

(a)

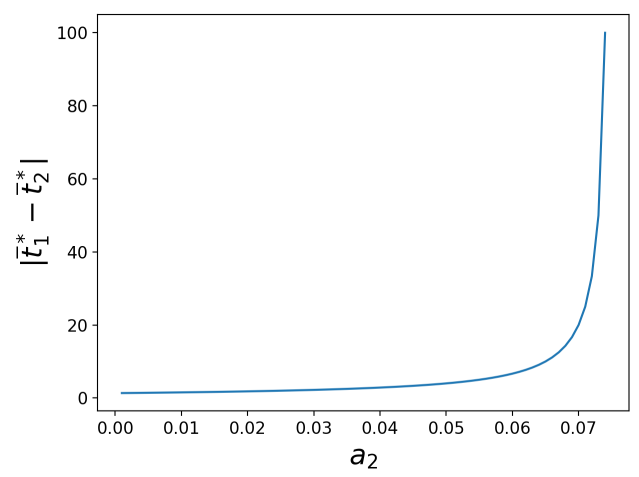

(c)

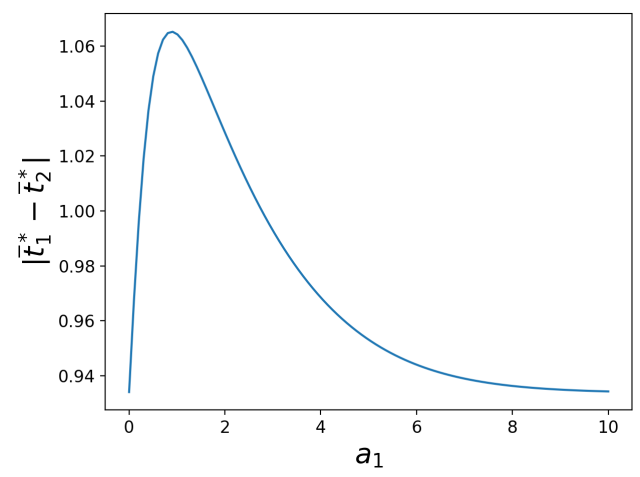

(b)

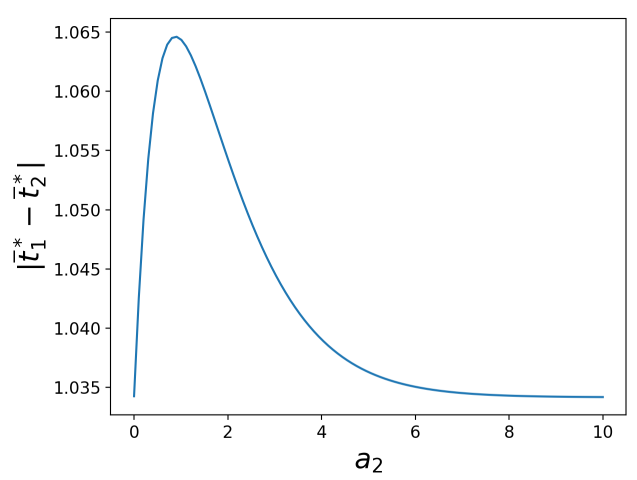

(d)

Figure 12: Influence of female choosiness in species (a)(b) $1 a_{1}$ and (c)(d) $2 a_{1}$ on the phenotypic distances between the focal species and the local mimicry rings, assuming (a)(c) weak or (b)(d) strong female choosiness and predator discrimination. Simulations were run assuming (a) $a_{2}=b=0.01$, (b) $a_{2}=b=1$, (c) $a_{1}=b=0.01$ or $(\mathrm{d}) a_{1}=b=1$. The default parameters values are as follows: $c_{R I}=0.01$, $d=0.02, \lambda_{1}=0.1, \lambda_{2}=0.1, N_{1}=10, N_{2}=20, s_{1}=0.0000025, s_{2}=0.0000025, t_{a 1}=0$, $t_{a 2}=1$ 


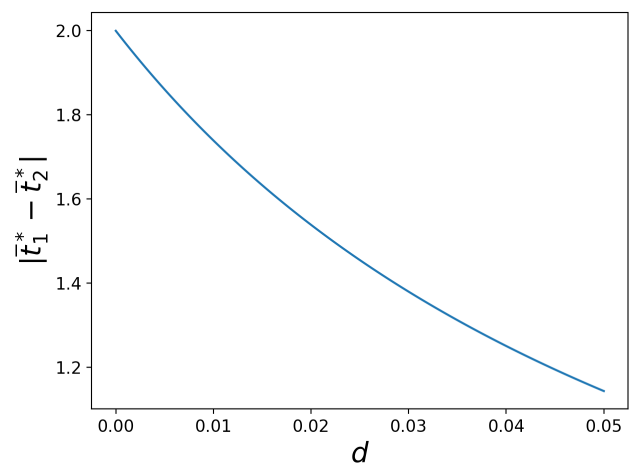

(a)

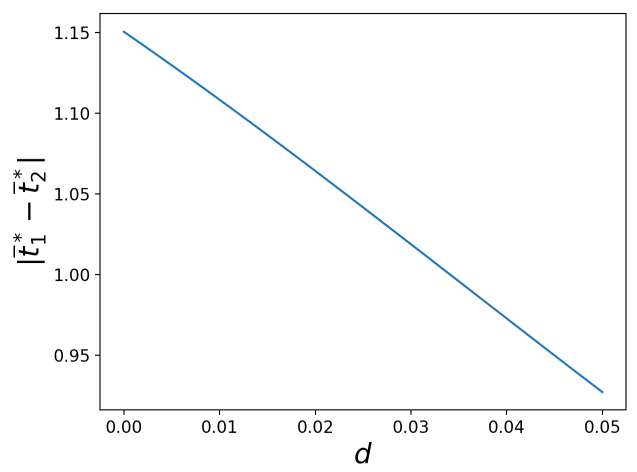

(b)

Figure 13: Influence of the strength of predation $d$ on the phenotypic distances between the focal species and the local mimicry rings, assuming (a) weak or (b) strong female choosiness and predator discrimination. Simulations were run assuming (a) $a_{1}=a_{2}=b=0.01$, (b) $a_{1}=a_{2}=b=1$. The default parameters values are as follows: $c_{R I}=0.01, \lambda_{1}=0.1, \lambda_{2}=0.1, N_{1}=10, N_{2}=20, s_{1}=0.0000025$, $s_{2}=0.0000025, t_{a 1}=0, t_{a 2}=1$. 


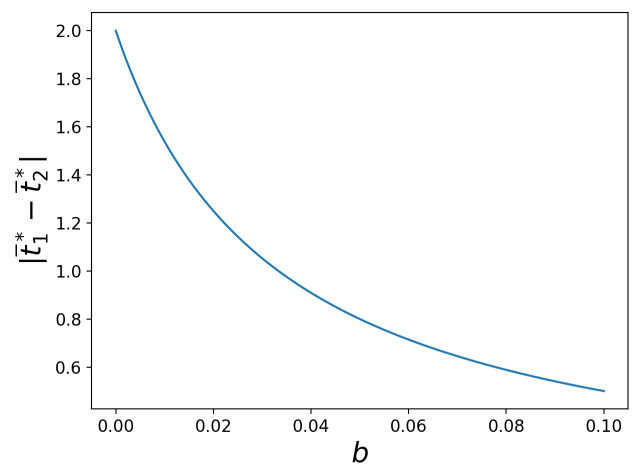

(a)

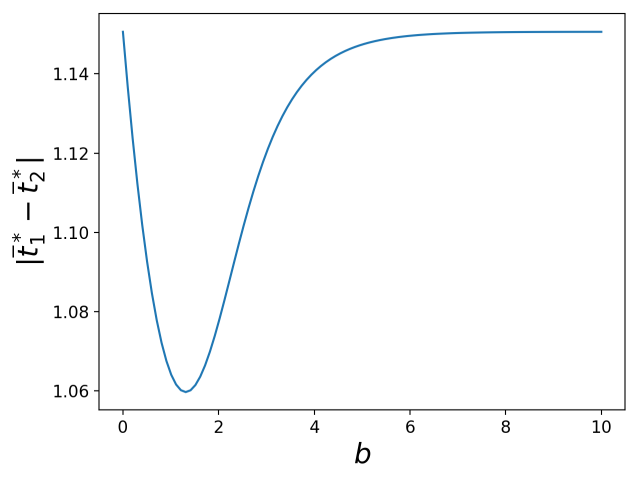

(b)

Figure 14: Influence of predator discrimination $b$ on the phenotypic distances between the focal species and the local mimicry rings, assuming (a) weak or (b) strong female choosiness and predator discrimination. Simulations were run assuming (a) $a_{1}=a_{2}=0.01$, (b) $a_{1}=a_{2}=1$. The default parameters values are as follows: $c_{R I}=0.01, d=0.02, \lambda_{1}=0.1, \lambda_{2}=0.1, N_{1}=10, N_{2}=20, s_{1}=0.0000025$, $s_{2}=0.0000025, t_{a 1}=0, t_{a 2}=1$. 


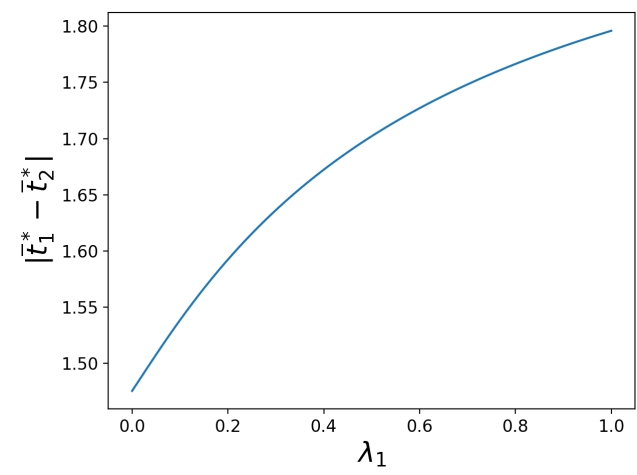

(a)

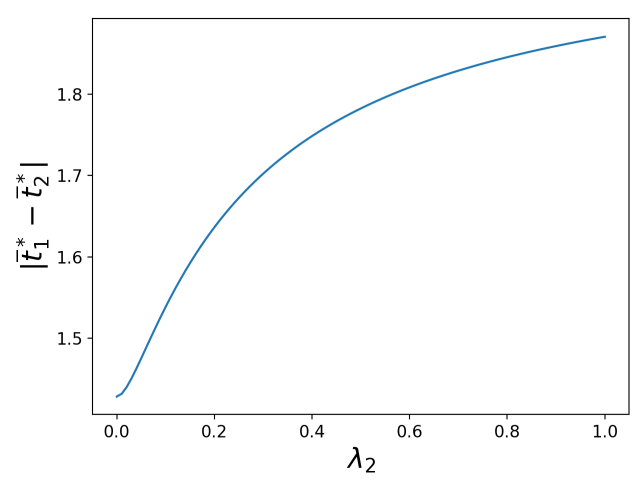

(c)

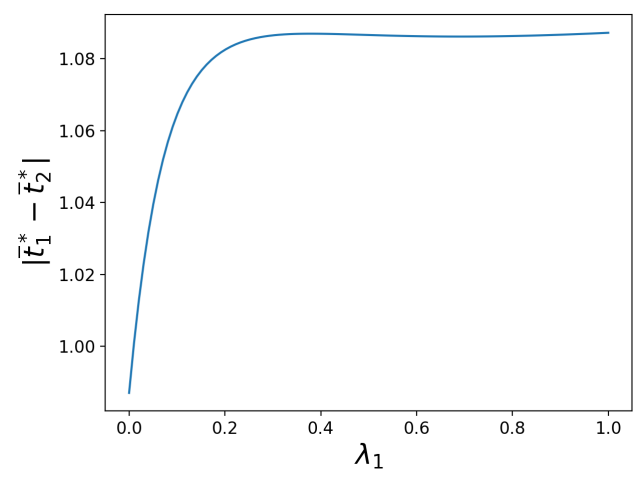

(b)

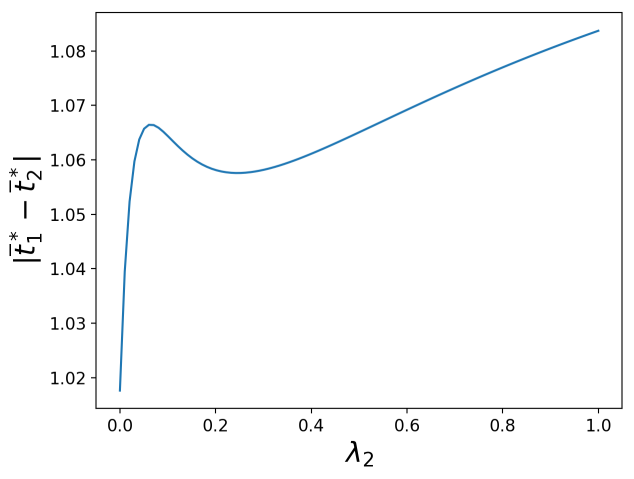

(d)

Figure 15: Influence of the defence level of (a)(b) species $1 \lambda_{1}$ and of (c)(d) species

$2 \lambda_{2}$ on the phenotypic distances between both species, assuming (a)(c) weak or (b)(d) strong female choosiness and predator discrimination. Simulations were run assuming (a)(c) $a_{1}=a_{2}=b=0.01,(\mathrm{~b})(\mathrm{d}) a_{1}=a_{2}=b=1$. The default parameters values are as follows: $c_{R I}=0.01, d=0.02, \lambda_{1}=0.1, \lambda_{2}=0.1, N_{1}=10, N_{2}=20$, $s_{1}=0.0000025, s_{2}=0.0000025, t_{a 1}=0, t_{a 2}=1$. 


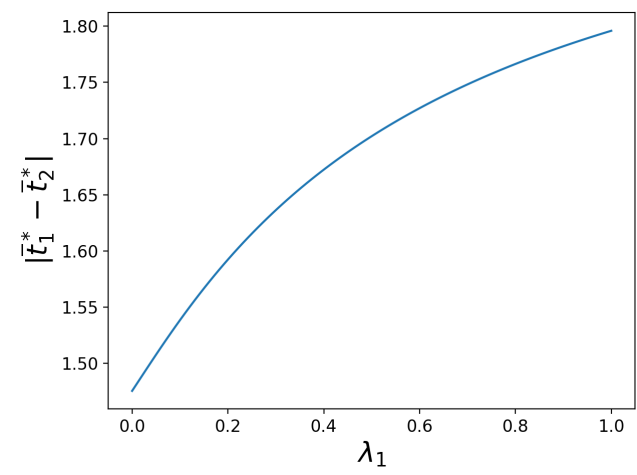

(a)

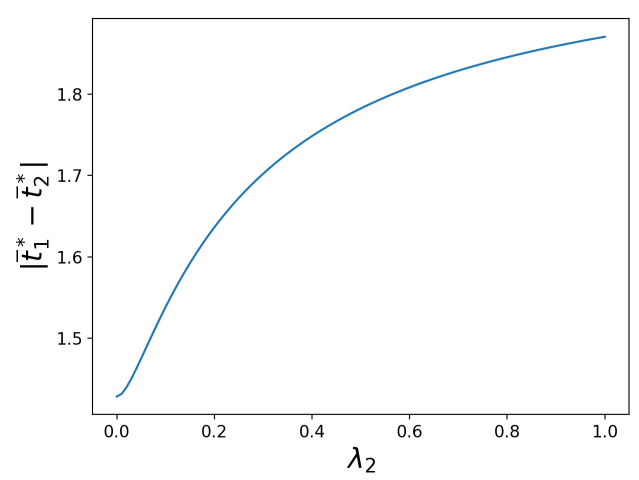

(c)

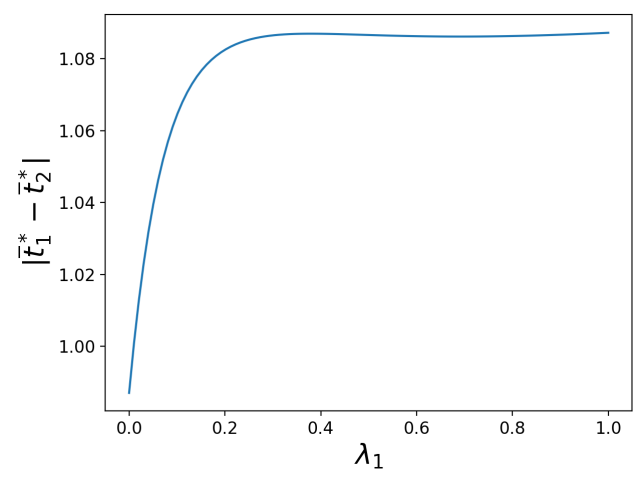

(b)

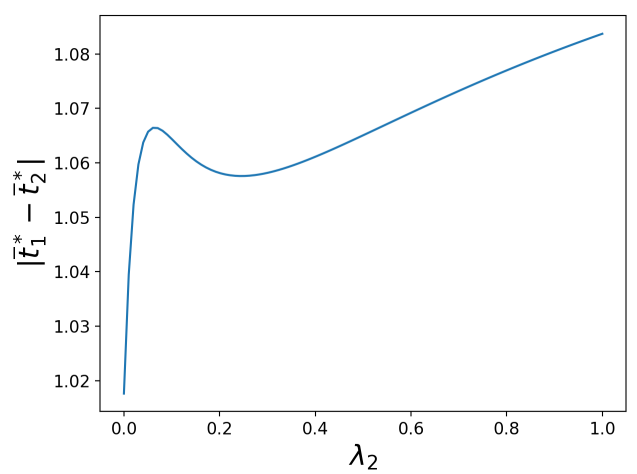

(d)

Figure 16: Influence of the defence level of (a)(b) species $1 \lambda_{1}$ and of (c)(d) species

$2 \lambda_{2}$ on the phenotypic distances between both species, assuming (a)(c) weak or (b)(d) strong female choosiness and predator discrimination. Simulations were run assuming (a)(c) $a_{1}=a_{2}=b=0.01,(\mathrm{~b})(\mathrm{d}) a_{1}=a_{2}=b=1$. The default parameters values are as follows: $c_{R I}=0.01, d=0.02, \lambda_{1}=0.1, \lambda_{2}=0.1, N_{1}=10, N_{2}=20$, $s_{1}=0.0000025, s_{2}=0.0000025, t_{a 1}=0, t_{a 2}=1$. 


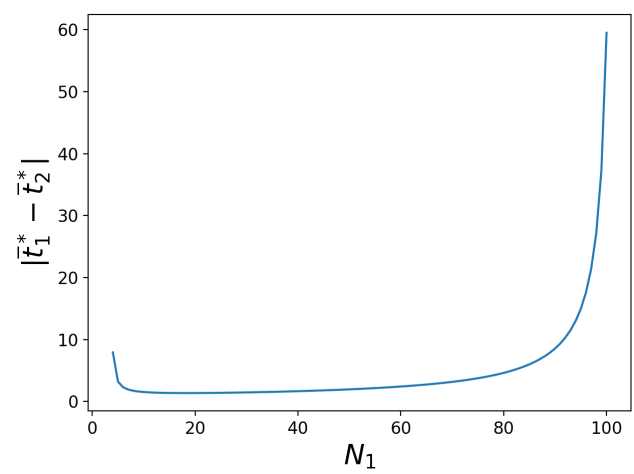

(a)

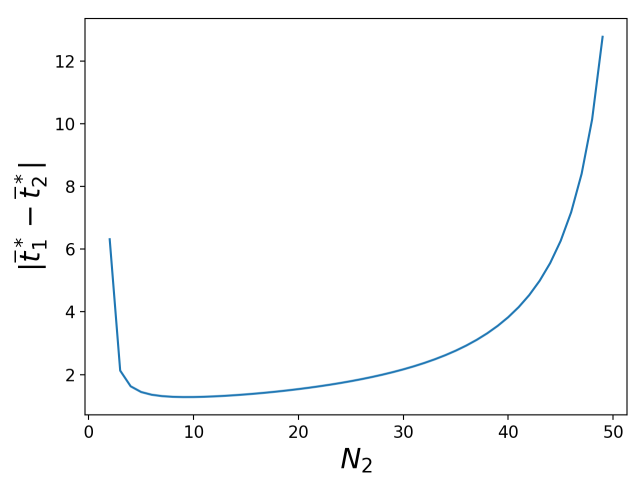

(c)

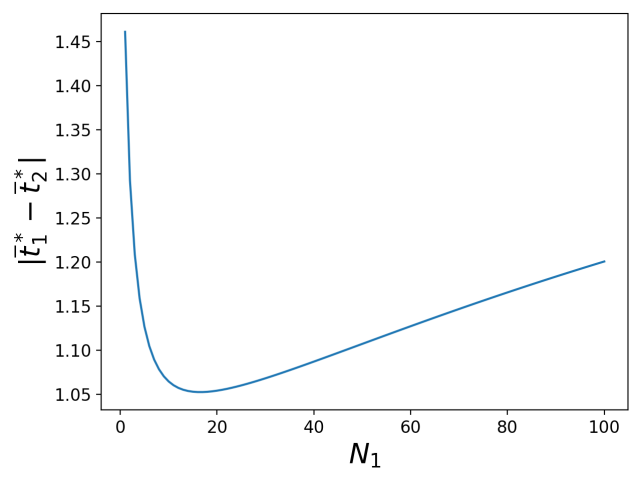

(b)

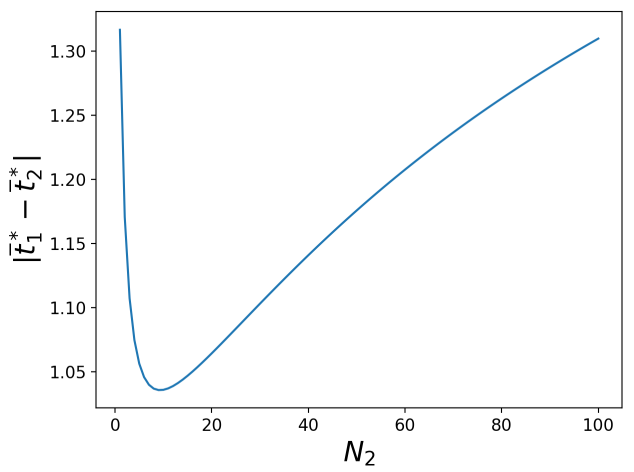

(d)

Figure 17: Influence of the density of (a)(b) species $1 N_{1}$ and of (c)(d) species 2 $N_{2}$ on the phenotypic distances between the focal species and the local mimicry rings, assuming (a)(c) weak or (b)(d) strong female choosiness and predator discrimination. Simulations were run assuming (a) $a_{1}=a_{2}=b=0.01$, (b) $a_{1}=a_{2}=$ $b=1$. The default parameters values are as follows: $c_{R I}=0.01, d=0.02, \lambda_{1}=0.1$, $\lambda_{2}=0.1, N_{1}=10, N_{2}=20, s_{1}=0.0000025, s_{2}=0.0000025, t_{a 1}=0, t_{a 2}=1$. 


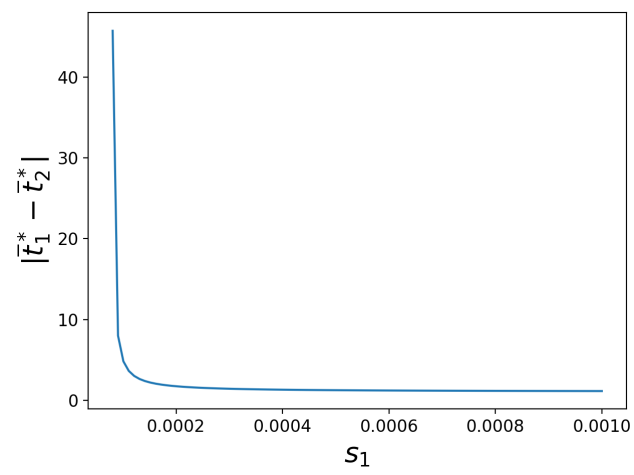

(a)

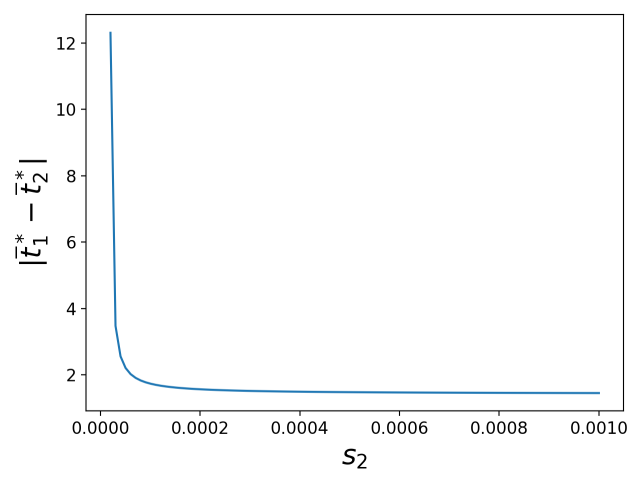

(c)

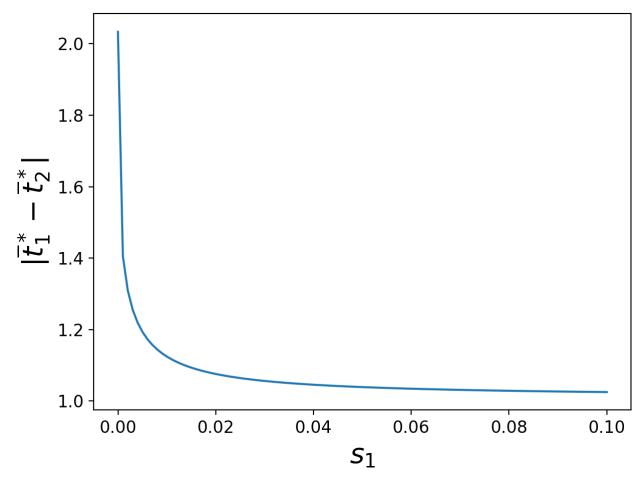

(b)

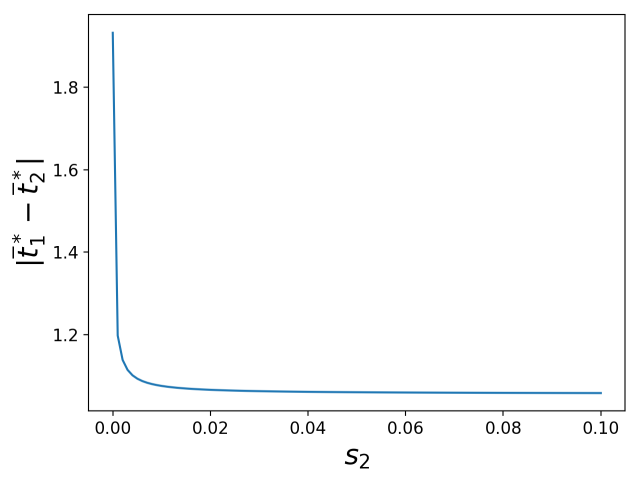

(d)

Figure 18: Influence of the strength of selective constraints in (a)(b) species 1 $s_{1}$ and in (c)(d) species $2 s_{2}$ on the phenotypic distances between the focal species and the local mimicry rings, assuming (a)(c) weak or (b)(d) strong female choosiness and predator discrimination. Simulations were run assuming (a)(c) $a_{1}=a_{2}=b=0.01$, (b)(d) $a_{1}=a_{2}=b=1$. The default parameters values are as follows: $c_{R I}=0.01, d=0.02, \lambda_{1}=0.1, \lambda_{2}=0.1, N_{1}=10, N_{2}=20, s_{1}=0.0000025$, $s_{2}=0.0000025, t_{a 1}=0, t_{a 2}=1$. 


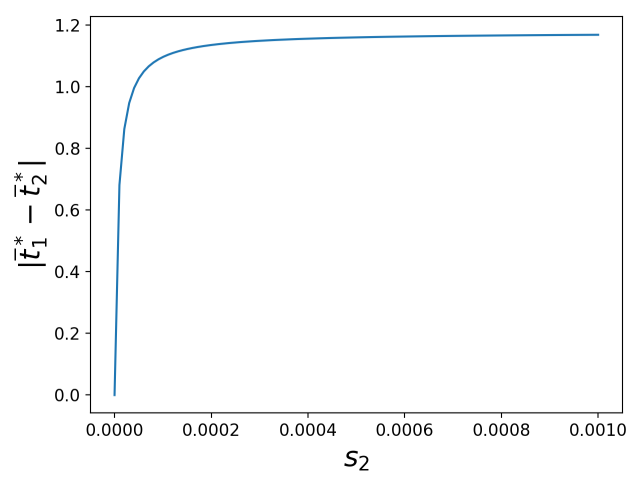

(a)

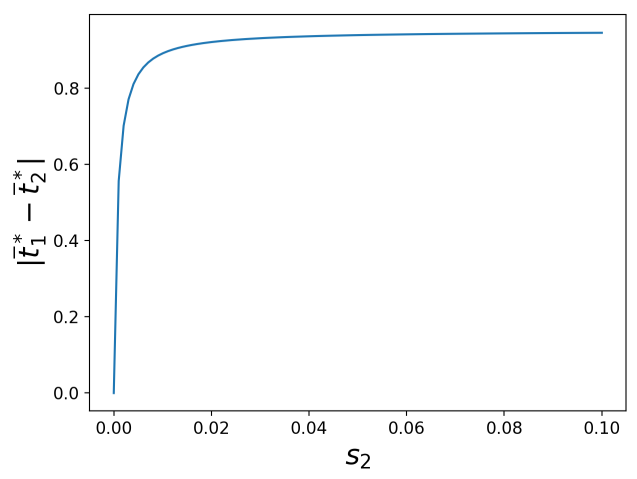

(b)

Figure 19: Influence of the strength of selective constraints in species $2 s_{2}$ on the phenotypic distances between the focal species and the local mimicry rings, when predation is great compare to RI, assuming (a) weak or (b) strong female choosiness and predator discrimination. Simulations were run assuming (a) $a_{1}=a_{2}=$ $b=0.01$, (b) $a_{1}=a_{2}=b=1$. The default parameters values are as follows: $c_{R I}=0.01$, $d=0.05, \lambda_{1}=0.1, \lambda_{2}=0.1, N_{1}=10, N_{2}=20, s_{1}=0.0000025, s_{2}=0.0000025, t_{a 1}=0$, $t_{a 2}=1$ 
550

554

556

560

552

558

\section{References}

N. H. Barton and M. Turelli. Natural and sexual selection on many loci. Genetics, 127

(1):229-255, 1991. ISSN 0016-6731. URL https://www.genetics.org/content/127/ $1 / 229$.

M. Kirkpatrick, T. Johnson, and N. Barton. General models of multilocus evolution.

Genetics, 161(4):1727-1750, 2002. ISSN 0016-6731. URL https://www.genetics.org/ content/161/4/1727.

R. Lande. Models of speciation by sexual selection on polygenic traits. Proceedings of the National Academy of Sciences, 78(6):3721-3725, 1981. ISSN 0027-8424. 10.1073/ pnas.78.6.3721. URL https://www.pnas.org/content/78/6/3721.

A. Pomiankowski and Y. Iwasa. Evolution of multiple sexual preferences by fisher's runaway process of sexual selection. Proceedings: Biological Sciences, 253(1337):173-181, 1993. ISSN 09628452. URL http://www.jstor.org/stable/49806. 